\title{
Ionizing Radiation and Life
}

\author{
Lewis R. Dartnell
}

\begin{abstract}
Ionizing radiation is a ubiquitous feature of the Cosmos, from exogenous cosmic rays (CR) to the intrinsic mineral radioactivity of a habitable world, and its influences on the emergence and persistence of life are wideranging and profound. Much attention has already been focused on the deleterious effects of ionizing radiation on organisms and the complex molecules of life, but ionizing radiation also performs many crucial functions in the generation of habitable planetary environments and the origins of life. This review surveys the role of CR and mineral radioactivity in star formation, generation of biogenic elements, and the synthesis of organic molecules and driving of prebiotic chemistry. Another major theme is the multiple layers of shielding of planetary surfaces from the flux of cosmic radiation and the various effects on a biosphere of violent but rare astrophysical events such as supernovae and gamma-ray bursts. The influences of CR can also be duplicitous, such as limiting the survival of surface life on Mars while potentially supporting a subsurface biosphere in the ocean of Europa. This review highlights the common thread that ionizing radiation forms between the disparate component disciplines of astrobiology. Key Words: Cosmic rays-Solar energetic particles-RadioactivityUltraviolet-Supernova-Gamma-ray burst-Magnetosphere-Panspermia-Biosignature. Astrobiology 11, 551-582.
\end{abstract}

\section{Introduction}

T HE SURFACE OF EARTH is a relatively benign environment with regard to ionizing particle radiation. The two major natural sources of ionizing radiation on a planetary body are the decay of radioactive isotopes present on the surface and the flux of cosmic rays from above. Cosmic rays $(\mathrm{CR})$ are charged subatomic particles that originate in space and are accelerated to high energies. Cosmic rays are a ubiquitous source of ionizing radiation throughout the Cosmos that impacts habitable worlds and the origin and maintenance of life in a plethora of different ways. This energetic particle radiation has been studied as a hazard of human space exploration or, more generally, as a radiation threat to organisms in the space environment or on a planetary surface (e.g., see recent reviews by Brack et al., 2010, and Horneck et al., 2010); but the influences of CR and planetary radioactivity upon life extend far beyond their direct radiobiological hazard. Ionizing radiation performs many crucial functions in astrobiology, including its role in star formation, driving astrochemistry and the synthesis of extraterrestrial organic molecules, and perhaps supporting a subsurface europan biosphere. The high flux of energetic electromagnetic radiation from astrophysical events such as gamma-ray bursts (GRB) is also considered in this review, and though radiation from these types of events is not termed CR, it can cause extreme ionization perturbations and seriously threaten planetary habitability.

Other authors have provided recent overviews of different aspects of ionizing radiation and astrobiology, and interested readers may like to consult some of these for further discussion. A study by Ferrari and Szuszkiewicz (2009) offers an excellent introduction for nonspecialists to the sources and interactions of CR and details of biological dosimetry. For reviews that focus on the nature of $\mathrm{CR}$ and their interaction with shielding matter see works by Amsler et al. (2008a, 2008b). Baumstark-Khan and Facius (2001) provided a very useful account of different sources of ionizing radiation, including radionuclide decay, and their biological effects, while Nelson (2003) focused on the cellular effects of CR strikes. Vainio et al. (2009) summarized the dynamics of the particle radiation environment at Earth, whereas Bazilevskaya et al. (2008) provided a more focused account of the ionization of Earth's atmosphere; and Bailer-Jones (2009) critically assessed claims that CR flux affects the terrestrial climate. Lammer et al. (2009) offered a broad overview of the influences on planetary habitability, including that of CR. Finally, the published conference proceedings by Akaboshi et al. (2000) present a selection of papers that address the roles of radiation in the origin and evolution of life. 
This paper reviews the current state of knowledge on the different roles of ionizing radiation in the emergence and maintenance of life, and so argues that CR act as a common thread between many of the disparate areas within astrobiology. Ionizing radiation is one of the major factors that reaches across the disciplines of astrobiology-linking life in a protected planetary environment to its cosmic context.

The review will not deal extensively with UV light, such as that produced by the broad spectrum stellar emission. Here, I am primarily concerned with the ionizing radiation environment produced by $\mathrm{CR}$, as well as extremely energetic electromagnetic radiation from astrophysical sources such as GRB. In addition, I discuss the role of the ionizing radiation field intrinsic to a habitable world generated by radionuclide decay in its crust or oceans where it is relevant to the origin and survival of life.

This review is not intended as a comprehensive or definitive resource and does not attempt to collate all related papers over the last few decades in fields as diverse as astrophysics, prebiotic chemistry, and planetary habitability. It is written as a broad overview for nonspecialists-an introductory resource to allow the reader to appreciate the wide-ranging influences that ionizing radiation exerts in astrobiology and to provide some key papers for follow-up reading.

I will begin by explaining the astrophysical sources of $C R$ and their deleterious effect on living organisms. I will then progress through the greatly diverse range of topics in astrobiology unified by $\mathrm{CR}$, explaining the role of $\mathrm{CR}$ in star formation, in the generation of elements needed by life, and in driving astrochemistry and the production of organic molecules. Cosmic rays also play a major role in the habitability of a world, and I will review how a planetary surface is shielded by the star's astrosphere, the planet's magnetosphere (if present) and atmosphere, and the influences of CR in forcing the climate or atmospheric chemistry. Cosmic ray ionization in the atmosphere may play an important role in the astrobiological potential of Venus and Titan, as well as on unshielded planetary bodies such as Mars, Europa, and meteorites, in the interest of panspermia. The next section deals with extreme, but irregular, ionizing radiation hazards, such as supernovae, GRB, and superflares, and the predicted effect on life, such as destruction of the ozone layer, global cooling, nitric acid rain-out, and intense UV pulse. Finally, I will review the possible roles of intrinsic radioactivity in planetary habitability and prebiotic chemistry and the occurrence of natural fission reactors. The structure of this review is as follows:

2. Cosmic Ray Sources

3. Biological Effects of Ionizing Radiation

4. The Role of Cosmic Rays in Star Formation

5. Role of Cosmic Rays in Biogenic Elements

6. Role of Cosmic Rays in Astrochemistry and Prebiotic Organic Molecules

7. Influence of Cosmic Rays on Planetary Habitability

7.1. Astrosphere

7.2. Planetary magnetosphere

7.3. Interaction of cosmic rays with atmosphere

7.4. Cosmic rays and climate forcing

7.5. Cosmic ray production of organics within the atmosphere

7.6. Venus atmosphere ionization
7.7. Titan atmosphere ionization

7.8. Sterilization of Mars' surface

7.9. Support of europan biosphere

7.10 Panspermia

7.11 Degradation of biosignatures

8. Extreme Radiation Events

8.1. Supernova destruction of the ozone shield

8.2. Gamma-ray bursts

8.3. Superflares

8.4. Global cooling

8.5. Nitric acid rain-out

8.6. Ultraviolet flash

8.7. Active galactic nuclei

8.8. Variation in the galactic cosmic ray flux

9. Intrinsic Planetary Radiation Environment

9.1. Radiation and prebiotic chemistry

9.2. Natural fission reactors

10. Conclusions

\section{Cosmic Ray Sources}

Beyond the shielding influence of Earth's magnetic field and atmosphere (see below), the space radiation environment is dominated by solar energetic particles (SEP; but also referred to as solar cosmic rays, SCR) and galactic cosmic rays (GCR), as illustrated in Fig. 1. SEP and GCR are summarized briefly here to provide context to the astrobiological effects of ionizing radiation discussed later; more complete reviews of $\mathrm{CR}$ can be found in studies by Ferrari and Szuszkiewicz (2009), Vainio et al. (2009), and Amsler et al. (2008a, 2008b).

Solar energetic particles, primarily protons, are accelerated by flares and coronal mass ejections to energies typically of tens to hundreds of megaelectronvolts. Particularly large particle events release an appreciable fluence up to around 1 $\mathrm{GeV}$ and cause significant ground level enhancements in CR intensity in detectors on Earth's surface (SEP were reviewed by Ryan et al., 2000, and acceleration mechanisms were reviewed recently by Giacalone and Kóta, 2006). The flux of these SEP is thus sporadic in nature and dependent on the 11-year solar activity cycle, one-half of the 22-year Hale cycle due to the periodic reversal of the Sun's magnetic field (Vainio et al., 2009). For certain considerations, such as the cumulative SEP fluence onto the lunar surface over millennia, time-averaged SEP spectra can be used (e.g., Kuznetsov et al., 2001, or Usoskin and Kovaltsov, 2006), but the total fluence, peak flux, and energy spectra vary greatly between individual SEP events (see comparison by Mewaldt, 2006), and events can last between a few hours and a week (reviewed by Vainio et al., 2009).

The peak flux of GCR particles, at around $500 \mathrm{MeV} /$ nucleon, is 3-4 orders of magnitude lower than the averaged SEP flux at $10 \mathrm{MeV}$ (e.g., Usoskin and Kovaltsov, 2006), but the power law tail of the CR spectra extends up to $10^{20} \mathrm{eV}$ at extremely low fluxes. The GCR spectrum is composed of $85 \%$ protons, $14 \%$ alpha (helium nuclei), and a small fraction of heavy ions (fully ionized atomic nuclei) and electrons. The current paradigm for GCR production is particle acceleration at the expanding shock of supernova remnants (Blandford and Eichler, 1987; Vainio et al., 2009), although details of the source matter and acceleration mechanisms are yet to be perfectly resolved (see the Butt, 2009, study for a recent 


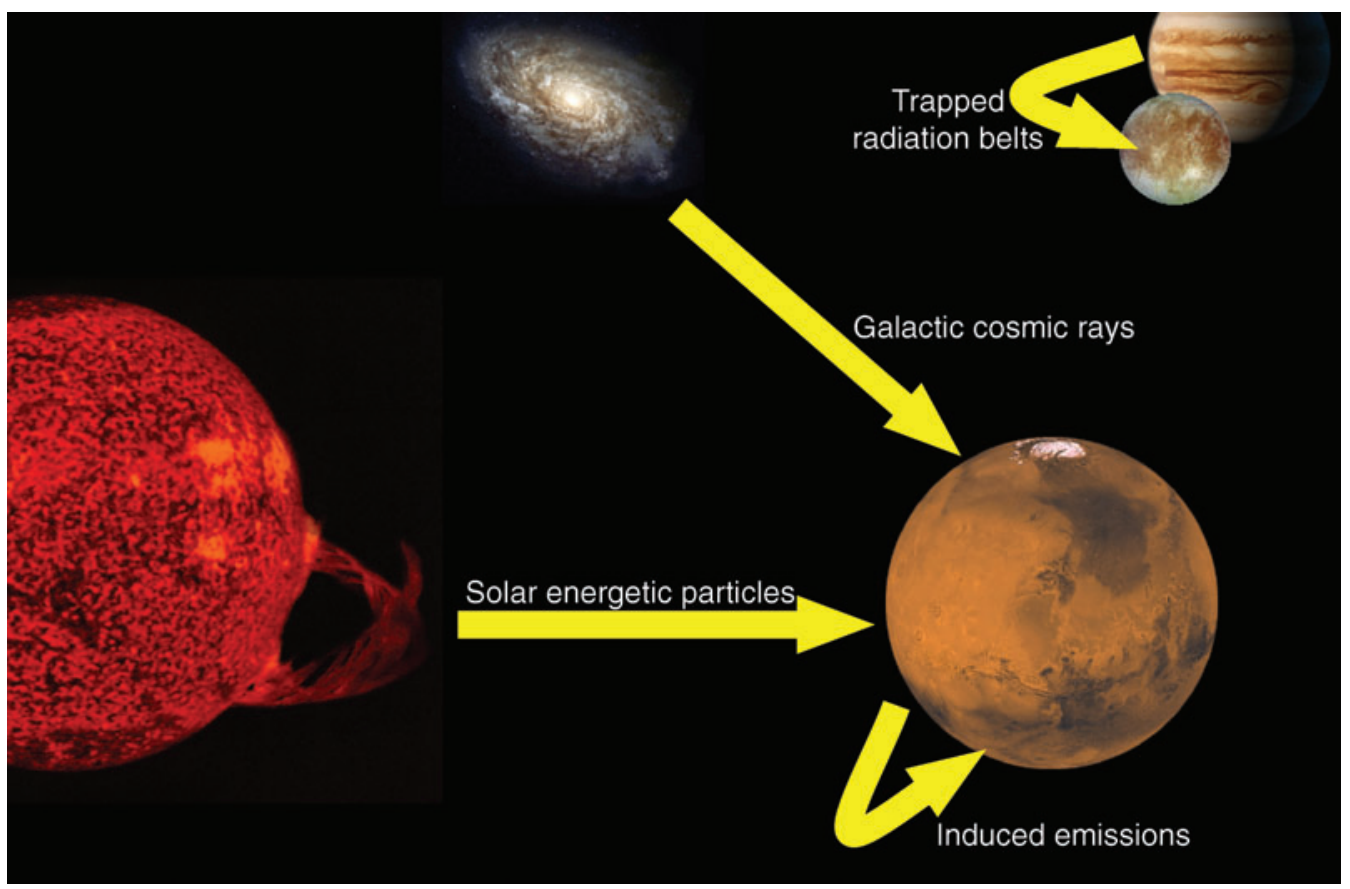

FIG. 1. Sources of cosmic radiation. Solar energetic particles are accelerated by coronal mass ejections, galactic cosmic rays by supernovae. Unshielded planetary surfaces, such as Mars, have a high albedo flux of induced emissions from CR primaries, and moons like Europa are embedded in the trapped radiation belt of their planet. Color images available online at www.liebertonline.com/ast

review of problems facing the standard model). The source of the ultrahigh-energy CR $\left(E>10^{18} \mathrm{eV}\right)$ is unknown and may be extragalactic (see review in Bauleo and Martino, 2009). GCR below about $1 \mathrm{GeV} /$ nucleon are modulated by the heliosphere so their flux is anticorrelated with the solar activity cycle (Klapdor-Kleingrothaus and Zuber, 2000). Thus, GCR and SEP represent two complementary populations of ionizing particles: GCR are present at a relatively constant low flux, but their spectra extend to very high energy levels, whereas SEP have a much higher flux at lower energies and are accelerated within sporadic events.

Figure 1 presents a diagram that summarizes the different CR sources relevant to this discussion: SEP, GCR, trapped particle belts, and induced emissions from CR interactions. A significant radiation source for potentially habitable moons orbiting within the magnetic field of their planet, such as Europa or Titan, are charged particles trapped in circular regions within the dipolar field, which in the terrestrial situation are known as the Van Allen belts (discovered by the Explorer I satellite, Van Allen, 1959, and reviewed recently by Benton and Benton, 2001). Induced emissions are comprised of albedo particles generated by nuclear reactions in CR secondary cascades (explained in more detail below) that backscatter upwards.

Energetic CR primary particles produce extensive showers of secondary particles in Earth's atmosphere, as shown in Fig. 2. When a GCR strikes an atmospheric nucleus, energetic secondary mesons (pions and kaons), nucleons, gammas, and nuclear fragments are produced, which then interact with other nuclei. Secondary mesons decay over a short timescale to produce muons, gamma rays, and electrons. The air shower is composed of a central "hard component" core of nuclear fragments within a spreading "soft component" cone of the electromagnetic cascade (Amsler et al., 2008a). The flux of secondaries builds with increasing shielding depth until it reaches a peak, known as the Pfotzer maximum, after which the average particle energy is below the threshold for new particle production and the cascade is steadily absorbed. In Earth's atmosphere, the Pfotzer maximum occurs at the altitude of $15-26 \mathrm{~km}$, depending on latitude and solar activity level (Bazilevskaya et al., 2008).

For exposed bodies such as Mars or asteroids, these secondary particle cascades occur in the top meters of subsurface regolith. Nuclear reactions within the secondary cascades generate species such as neutrons and gamma rays that are scattered back out of the subsurface. These backscattered, or albedo, particles can significantly enhance the planetary surface radiation environment (Clowdsley et al., 2001) and can be detected by remote sensing instruments to allow mapping of surface composition. For example, albedo neutrons measured by instruments aboard the Mars Odyssey orbiter provided information on the distribution of hydrogen and, thus, presumably water ice deposits in the martian near surface (Feldman et al., 2002; Mitrofanov et al., 2002).

For more detail on the production and dynamics of SEP and GCR and their interactions with shielding matter (be it planetary atmospheres, surface, or spacecraft pressure hulls), interested readers are directed to recent reviews provided by, for example, Amsler et al. (2008b), Bazilevskaya et al. (2008), and Ferrari and Szuszkiewicz (2009).

\section{Biological Effects of lonizing Radiation}

The ionizing radiation field produced by SEP and GCR is harmful to life through direct and indirect mechanisms (Nelson, 2003). Direct damage occurs when deposited energy 


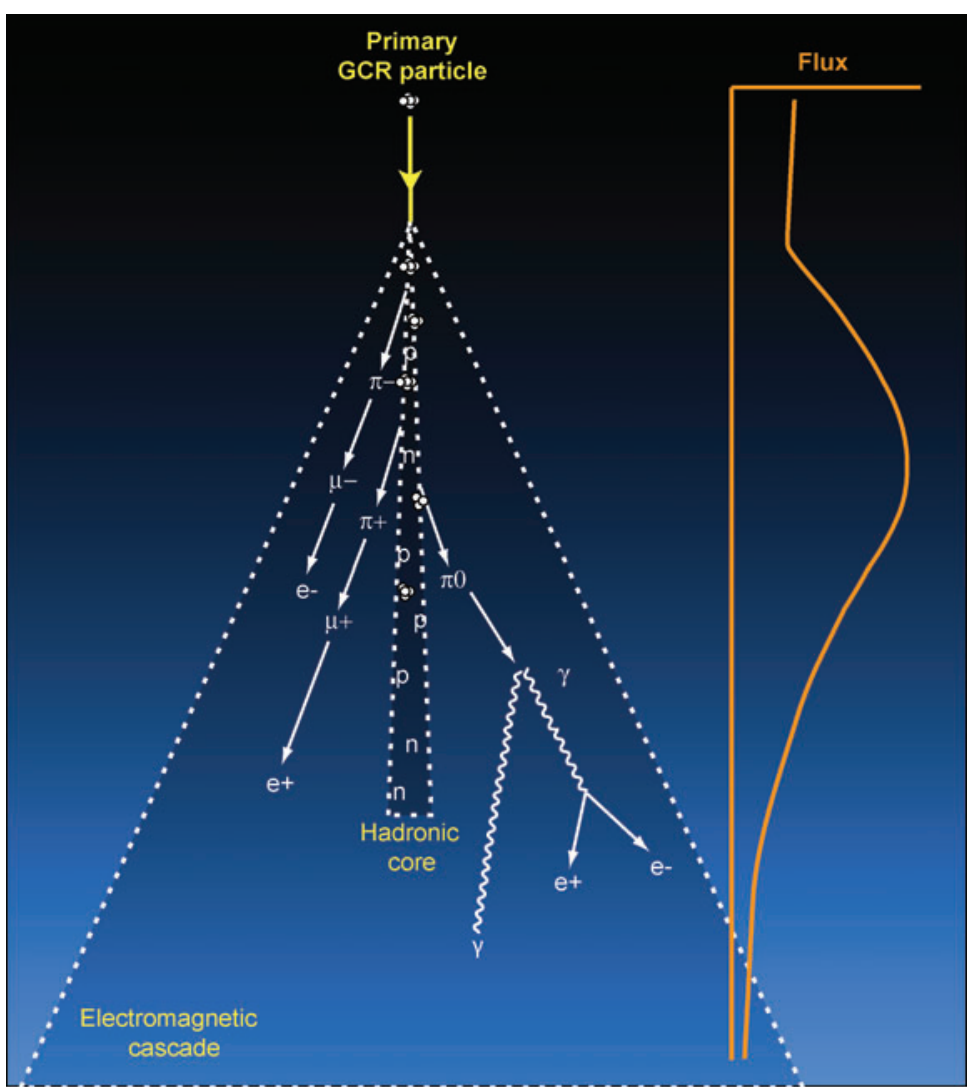

FIG. 2. The extensive cascade of secondary particles, or air shower, generated by an energetic primary CR striking a nucleus in Earth's upper atmosphere. The central hadronic core is composed of neutrons, protons, and nuclear fragments propagating essentially straight ahead, within the spreading electromagnetic cone of pions and muons and their decay products electrons and gamma-ray photons. Particle flux builds to a peak, known as the Pfotzer maximum, before being increasingly absorbed. Color images available online at www.liebertonline.com/ast excites electrons within biomolecules, which leads to ionization or radiolysis. However, radiation primarily interacts with water, as cells are composed of $40-70 \%$ water and bacterial spores $20 \%$, which leads to the indirect mechanism of radiation damage. Irradiated water undergoes ionization and radiolysis, resulting in the production of hydrated free electrons $\left(\mathrm{e}_{\mathrm{aq}}^{-}\right)$and highly reactive species with unpaired electrons, and free radicals, such as $\mathrm{H} \bullet$ and $\bullet \mathrm{OH}$, or their recombination products such as hydrogen peroxide (Kiefer, 1990). The major reaction pathways of irradiated water are shown in Fig. 3. These radiogenic products are freely diffusible and migrate from their site of production to attack and oxidize biomolecules such as DNA and proteins. If the irradiated water is oxygenated, greater yields of hydrogen peroxide and the hydroxyl radical are produced, and the indirect mechanism of biomolecule damage is enhanced (BaumstarkKhan and Facius, 2001). While ionizing radiation is harmful to life itself, the radiolysis and free-radical chemistry driven by $\mathrm{CR}$ on the surface ice of Europa may help maintain an oceanic ecosystem below. This topic is explored in Section 7.9.

Organisms exhibit varying degrees of resilience to ionizing radiation, with the Gram-negative (the classification of bacteria with a kind of cell wall that does not retain crystal violet dye in the Gram staining protocol) nonsporulating bacterium Deinococcus radiodurans being the most radioresistant organism known (Minton, 1994; Battista, 1997; Cox and Battista, 2005). Gamma-irradiated populations of D. radiodurans can survive a dose of $5 \mathrm{kGy}$ without measurable loss of viability despite massive DNA fragmentation, and 1\% survival is still found after $10 \mathrm{kGy}$ (Cox and Battista, 2005). The desiccated endospores of bacteria such as Bacillus subtilis are also very radiation resistant (Baltschukat and Horneck, 1991; Setlow, 1994; Moeller et al., 2010), and after 3 kGy of X-rays, $10^{-2}$ spore viability is still found (Baumstark-Khan and Facius, 2001). For comparison, non-radioresistant bacteria such as E. coli exhibit only of the order $10^{-6}$ population survival after $1 \mathrm{kGy}$ (Battista, 1997). This remarkable resilience has long been believed to be primarily due to more efficient enzymatic repair of DNA double strand breaks (Battista et al., 1999). More recently, however, evidence has been mounting that other factors such as high intracellular manganese concentrations and protection of proteins from oxidation (Daly et al., 2004; Ghosal et al., 2005; Daly, 2009) are key determinants of $D$. radiodurans' radiation survival. Due to this extreme radioresistance, as well as resilience to desiccation, hydrogen peroxide, and UV radiation, D. radiodurans is commonly used as a model strain for microorganisms able to survive on the martian surface (e.g., Richmond et al., 1999; Horneck, 2000; Pavlov et al., 2002; Dartnell et al., 2007a; de La Vega et al., 2007). More recently, strains of a hyperthermophilic archaeon Thermococcus, isolated from a submarine hydrothermal vent environment, have been found with a radiation resistance approaching that of $D$. radiodurans (Jolivet et al., 2003, 2004).

The effects of ionizing radiation on the structure of biomolecules and the survival of organisms has been well covered elsewhere, so only a brief summary has been provided here to offer context for the following discussion on astrobiological aspects of ionizing radiation that have not yet received due coverage. For more complete reviews of space radiobiology, the interested reader is referred to reviews by Baumstark-Khan and Facius (2001) or Nelson (2003) and 


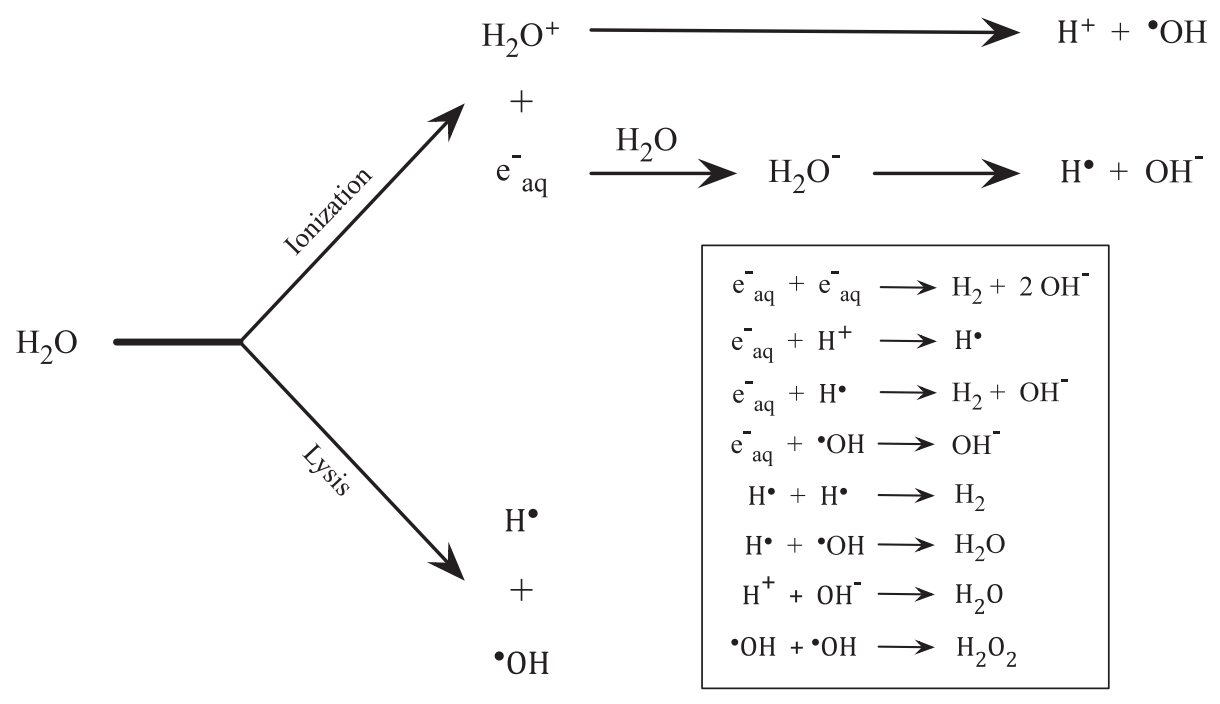

FIG. 3. The reaction pathways of ionization and lysis of irradiated anoxic water, and the ensuing free-radical chemistry. The products of radiolyzed water molecules include the highly reactive, and biologically damaging, water solvated electron ( $\left.\mathrm{e}_{\mathrm{aq}}^{-}\right)$, hydroxyl radical $(\bullet \mathrm{OH})$, and recombination products such as hydrogen peroxide $\left(\mathrm{H}_{2} \mathrm{O}_{2}\right)$. These diffusible species constitute an indirect mechanism of radiation damage. Figure constructed from information summarized in Pimblott and LaVerne (1992) and Baumstark-Khan and Facius (2001).

papers that consider more specifically the hazard posed by CR to human space exploration (Todd, 2003; NCRP, 2006).

Ionizing radiation can induce mutations and act as a selective filter for survival, and so it can potentially influence both of these drivers of natural selection and evolution of life (Committee on the Origins and Evolution of Life, 2005). Except for transient extreme ionization events (see Section 8), Earth's surface has been well shielded from CR throughout its history by a thick atmosphere (Svensmark, 2006, estimated variation in CR flux over the planet's history; discussed here in Section 8.8), and although organism exposure from environmental radionuclides has decreased by a factor of around 5 since primordial Earth, this has largely been counteracted by rising oxygen levels and consequent enhancement of radiation damage (Karam and Leslie, 1999; Karam et al., 2001). Surface radiation contributes to mutagenesis rates along with a variety of other causes, including replication errors and DNA damage from UV, chemical oxidants, and desiccation (Makarova et al., 2001). The rate of DNA damage wreaked by reactive oxygen species (such as hydroxyl radicals or hydrogen peroxide) generated by an aerobic cell's own metabolism is, however, estimated to be orders of magnitude greater than that due to current background levels of ionizing radiation (Imlay, 2003).

While this may be true for background levels of ionizing radiation, stochastic extreme cosmic ionization events, such as a nearby supernova, could occasionally and transiently raise the mutation rate of exposed organisms. The question is whether such pulses of hypermutation would be generally deleterious to a population, potentially resulting even in extinction, or could perhaps prove beneficial and enhance evolution. Smith et al. (2004a) argued that a fluctuating radiation environment and hypermutation events would produce a more diverse adaptive landscape and offer natural selection greater allelic variation within a population to operate upon. They thus concluded that habitable worlds with a more strongly fluctuating radiation environment than
Earth may in fact experience accelerated evolution and development of complexity. Smith et al. (2004a) presented an in-depth account of the argument, as did Scalo et al. (2007, and references therein), who considered the possibility of accelerated evolution on planets around M-class stars driven by a fluctuating radiation environment. There is genomic evidence from bacteria residing inside eukaryotic cells (endocellular symbionts) that increased mutation rates can indeed drive faster evolution (Itoh et al., 2002). Organisms with mutator phenotypes (exhibiting elevated mutation rates) are known in nature, and though advantageous in the short term when colonizing or adapting to a new environment, they are generally maladaptive due to the accumulation of deleterious mutations (e.g., Giraud et al., 2001). The concept of adaptively variable mutation rates and the ability to evolve, or "evolvability," of organisms was reviewed by Radman et al. (1999) and Pigliucci (2008). However, there is no experimental evidence that explicitly links heightened radiation exposure and subsequent hypermutation to an enhanced rate of evolution. Indeed, bacteria chronically exposed to high doses of ionizing radiation were found to adapt to exhibit greater radiation resistance and thus maintain a lower mutation rate (Ewing, 1995, 1997).

While background CR currently pose no significant hazard to the survival of life on Earth's surface, cosmic radiation may well play a decisive role in the terrestrial biosphere during acute astrophysical events (see Section 8), or over the longer term on planets with less shielding, such as Mars (see Section 7.8), or habitable planets orbiting other classes of stars (see Section 7.2). There is also the possibility that a high flux of ionizing radiation could support an extraterrestrial ecology on radiogenic products (see Section 7.9). These biological ramifications of ionizing radiation will be treated later, but for now this review will discuss the ways in which CR influence the creation of habitable environments for life, from their role in the formation of stars to the synthesis of biogenic elements and the organic precursor molecules of biochemistry. 


\section{The Role of Cosmic Rays in Star Formation}

Cosmic rays pervade the Galaxy and ionize the atoms and molecules of the interstellar medium (and are the dominant source of ionization in deep regions where UV radiation cannot penetrate to photoionize; McKee and Ostriker, 2007; Padovani et al., 2009). When charged, these interstellar gas particles are influenced by galactic magnetic fields and through ion-neutral collisions with other particles affect the dynamics of the entire gas cloud. So GCR help tie the behavior of interstellar gas to the ambient magnetic fields of the Galaxy. Magnetic fields stabilize interstellar molecular clouds against gravitational collapse, which regulates the collapse of protostellar clumps (Zweibel and Heiles, 1997) and sheds angular momentum of star-forming cloud cores and protostellar disks by magnetic braking (Desch and Mouschovias, 2001; Allen et al., 2003; Larson, 2003). The ramification of this is that, alongside UV photoionization, ionization by CR plays a role in star formation (reviewed recently by McKee and Ostriker, 2007; Larson, 2010) and is particularly significant deep in star-forming clouds or in the outer regions of protostellar disks. Cosmic rays also act to heat the interstellar medium (ISM), which raises the gas pressure and further increases the stability against gravitational collapse (Zweibel and Heiles, 1997; Padovani et al., 2009). This coupling of interstellar gas to galactic magnetic fields by CR ionization is therefore thought to exert control over the mass distribution of star formation (Rees, 1987).

Thus, it could be argued that each step of the cycle of stellar evolution in the Galaxy is linked together by $\mathrm{CR}$, as illustrated in Fig. 4. Supernovae shock fronts accelerate the particles of $\mathrm{CR}$, which are contained by the galactic magnetic field. These trapped CR act to ionize the interstellar gas clouds so that they become responsive to the magnetic fields. This process moderates star formation, with massive stars ending their evolution in supernovae, thus completing the cycle.

Creation of the first stars, termed Population III stars, in the primordial Milky Way and other galaxies would have necessitated different mechanisms from modern star birth. Such early stars must have formed from metal-free primordial gas in the absence of dust grains (and thus without efficient radiative cooling promoting contraction) and without ionization of the gas by $C R$ or regulation by galactic magnetic fields. Modeling studies indicate that the earliest stars in the Universe were thus probably very massive; in excess of 100 times solar mass (Abel et al., 2002; Bromm et al., 2002; Tan and McKee, 2004, 2008; and recently reviewed by Bromm et al., 2009). Observational evidence for these early massive stars is anticipated over the coming decade (Bromm et al., 2009), aided by the James Webb Space Telescope. The luminous supernova SN 2007bi is believed to have been a pair-instability supernova, predicted by theory to result only from stars greater than 140 solar mass, which suggests the formation of very massive stars in modern dwarf galaxies through similar mechanisms to the first stars in the Universe (Gal-Yam et al., 2009).

After the first generation of supernovae, interstellar space would have become pervaded with GCR and enriched with heavier elements. Not all the heavy elements of the Periodic Table are produced by stellar or supernova nucleosynthesis, however, and the formation of several key elements can only be explained by CR, as discussed in the next section.

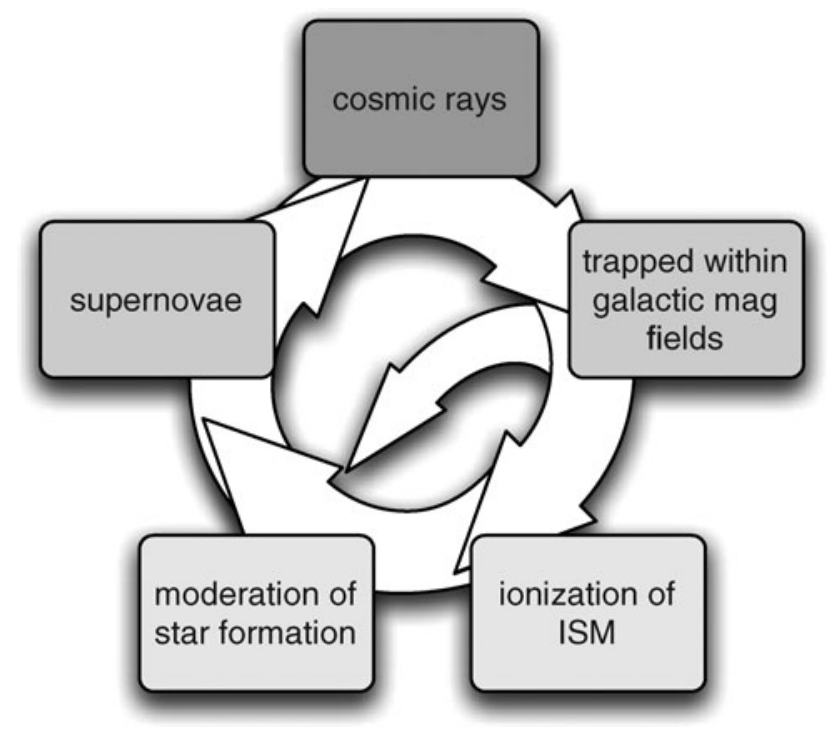

FIG. 4. The key role that CR play in the cycle of star formation. Ionization of the ISM by CR moderates star formation and, thus, the rate of occurrence of supernovae, which accelerate ions to form the GCR background. The galactic magnetic field serves to trap CR particles within the Galaxy and performs several crucial functions in star formation, as discussed in the text.

\section{Role of Cosmic Rays in Biogenic Elements}

The lightest isotopes, $\mathrm{H}, \mathrm{H}-2, \mathrm{He}-3, \mathrm{He}-4$, and a small amount of Li-7, were produced by nucleosynthesis during the Big Bang. The heavier elements are produced by nucleosynthesis reactions in stellar fusion, such as the triple alpha process, which fuses three helium nuclei into carbon; and elements beyond iron are generated by supernova nucleosynthesis. The cosmic abundance of the light elements lithium, beryllium, and boron ( $\mathrm{LiBeB})$ is, however, initially difficult to explain as they are destroyed by the hightemperature environments of the early Universe and stellar interiors (Prantzos et al., 1993). These LiBeB elements play important roles in biology, so the mechanism of their generation is important here.

A clue as to the origin of these fragile, light elements is provided by the observation that the abundance of this triplet relative to hydrogen is many orders of magnitude greater in GCR compared to the Solar System composition, as shown in Fig. 5.

The source of these fragile LiBeB elements was proposed 40 years ago (Reeves et al., 1970; Meneguzzi et al., 1971) to be nuclear spallation reactions between the energetic protons and alpha particles of the GCR and carbon, nitrogen, and oxygen nuclei of the low-density ISM. Another source was suggested to be fast carbon and oxygen nuclei GCR particles that impact the ambient hydrogen and helium (Reeves, 1994; Zhai, 1995; Vangioni-Flam and Casse, 2000). One particular problem in fitting such models to observed isotopic ratios is the fact that the metallicity of the ISM has increased over galactic history (through stellar and supernova nucleosynthesis) and modeled GCR LiBeB synthesis calculations either underproduce $\mathrm{Be}$ and $\mathrm{B}$ at low metallicities or overproduce $\mathrm{Li}$ (mostly produced by $\mathrm{He}+\mathrm{He}$ reactions) (Prantzos et al., 
FIG. 5. Comparison of the elemental composition of the Sun (filled black) and the GCR (white). Both GCR and Solar System abundances are normalized to $\mathrm{Si}=10^{3}$. The solar abundance of lithium, beryllium, and boron is very low and greatly enhanced in the GCR spectrum due to spallation of carbon, nitrogen, and oxygen nuclei. A similar GCR enhancement is seen in the elements Sc-Mn from spallation of iron but is not discussed further here. Figure constructed from data in Israel et al. (2004). Color images available online at www.liebertonline.com/ast

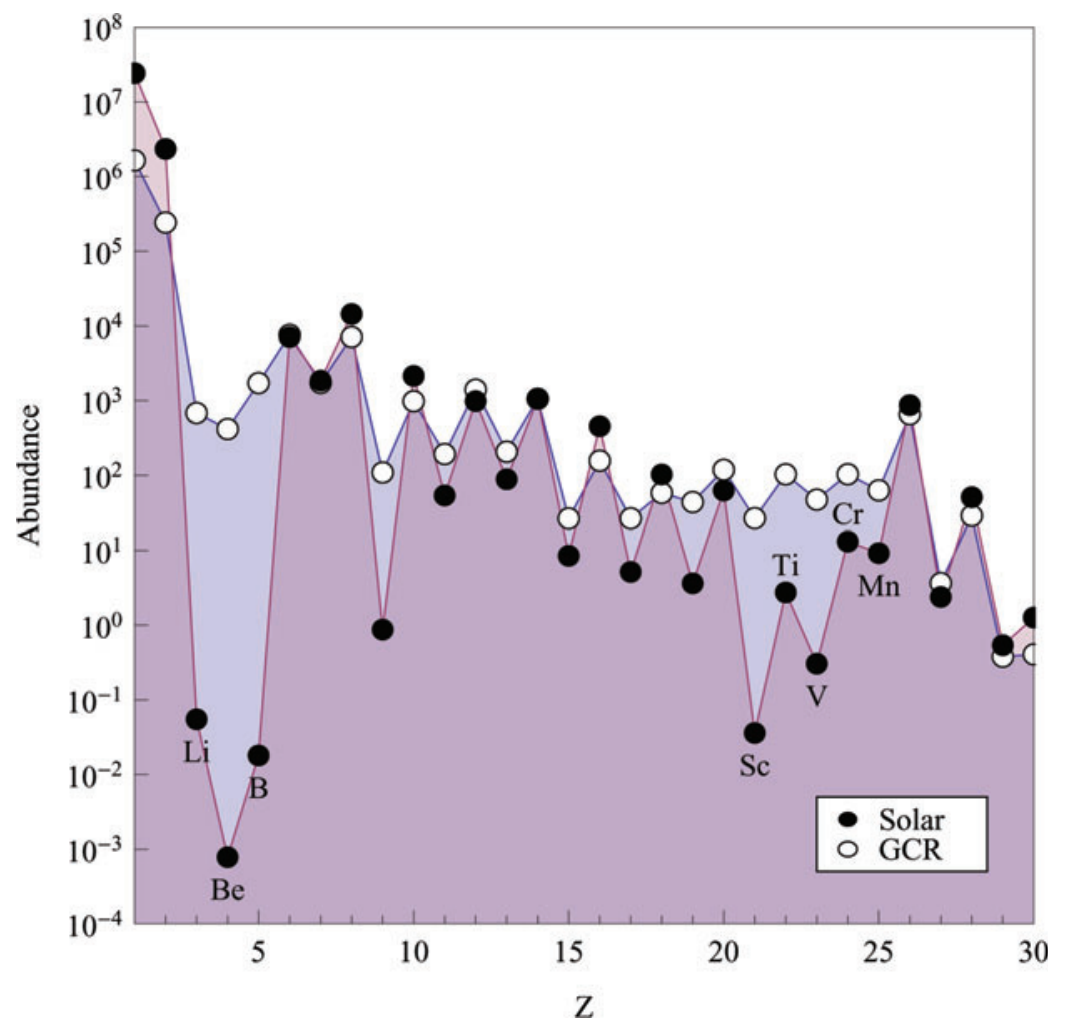

1993). Proposed explanations for the inconsistencies between predicted and observed ratios of LiBeB isotopes include refinements to the sources of CR (Vangioni-Flam and Casse, 2000) or additional sources of $\mathrm{LiBeB}$ isotopes, such as the hot outer envelopes of asymptotic giant branch stars ("red giants;" Prantzos, 2006). Ramaty et al. (2001) and Prantzos (2007) presented comprehensive reviews of the development of theories to explain GCR production of the LiBeB elements during the evolution of the Galaxy.

These CR-generated LiBeB elements, incorporated into new stars and planets from collapsing interstellar clouds, play important roles within biology. The elements employed by terrestrial life are, on the whole, those that are most abundant in Earth's crust, ocean, and atmosphere. Lithium and beryllium are less abundant at Earth's surface than alternative elements such as sodium, potassium, magnesium, and calcium, all of which are able to perform the same chemical functions (Fraústo da Silva and Williams, 2001). So lithium and beryllium are not essential elements for terrestrial life and can in fact show toxicity at high concentrations. They form small ions similar to $\mathrm{Mg}^{2+}$, and so they can interfere with biochemical processes through their interaction with phosphate-containing molecules (Fraústo da Silva and Williams, 2001). Beryllium has also been implicated in enzyme inactivation and malfunction (Wackett et al., 2004).

Boron, the third element of the CR-produced LiBeB triplet, is, however, vital for the proper biochemical functionality of many diverse branches of terrestrial life (reviewed by Bolaños et al., 2004). For example, boron is required by cyanobacteria for nitrogen fixation, plants employ borate esters in keeping their tissues flexible (Wackett et al., 2004), and boron is also considered a necessary ultratrace element in human metabolism (Nielsen, 1998). The main biochemical functionality of boron is believed to be its ability to stabilize molecules with cis-diol groups (where both $-\mathrm{OH}$ functional groups are on the same side; Bolaños et al., 2004), such as cyclic ribose (Ricardo et al., 2004), and it is for this ability (Benner et al., 2010) that boron has also been hypothesized to have played a crucial role in prebiotic chemistry and the origin of life. The conditions for an RNA world that led to cellular life have been doubted, as the prebiotic synthesis of ribose and other pentose sugars is difficult to explain. The presence of borate minerals, however, stabilizes the 1,2-diol structure of these pentoses and allows them to accumulate under prebiotic conditions (Ricardo et al., 2004; Benner et al., 2010). Grew et al. (2011) discussed the availability of borate minerals around 4 billion years ago to support an RNA world, and concluded that it critically depends on an early differentiation of a granitic continental crust.

Schulze-Makuch and Irwin (2008) even discussed the possibility of boron forming the basis of an extraterrestrial biochemistry. Boron, like carbon, has a remarkable propensity for forming covalent molecular compounds, and boronnitrogen compounds exhibit physical and chemical properties similar to alkanes and aromatic hydrocarbons. Boron compounds dissolve well in nitrogen and ammonia, so these could serve as the biosolvent for low-temperature boronbased life (Schulze-Makuch and Irwin, 2008).

So CR are crucial for the creation of the light LiBeB elements that are all biologically active, and boron is vital for biochemistry and may have played a key role in the origin of life. As well as driving nuclear reactions to generate elements crucial for biology, CR play a major role in the interstellar synthesis of organic molecules for life, as will be seen in the next section. 


\section{Role of Cosmic Rays in Astrochemistry and Prebiotic Organic Molecules}

Despite the low densities and temperatures, a great deal of complex chemistry occurs within interstellar dense molecular clouds. Cosmic rays, as well as UV photoionization, drive these reactions, and many of the molecules created here are crucial for biology (for example, see recent reviews by Ehrenfreund and Charnley, 2000; Charnley et al., 2002; and Snow and Bierbaum, 2008). Much of this "astrochemistry" is thought to depend on surface catalysis on interstellar dust grains (as reviewed by Williams and Herbst, 2002). Reactants and products are in a dynamic equilibrium of adsorption onto grain surfaces and release back into the gas phase through desorption induced by UV and CR (Öberg et al., 2007). Heating of these dark molecular clouds, and thus the thermal energy required for surface jumping and chemistry, is provided by CR. Cosmic ray ionization also drives the ionmolecule chemical reactions for atoms whose ionization potential is greater than that of hydrogen and, in particular, initiates the reactions of nitrogen- and oxygen-containing molecules (Federman et al., 1996). Ultraviolet photoionization also plays a role in driving astrochemistry in the diffuse ISM. Within cold dark molecular clouds $(\sim 10 \mathrm{~K})$, however, the dense dust absorbs UV photons, and only CR can penetrate inside (reviewed well by Dalgarno, 2006). Cosmic ray excitation of molecular hydrogen within the clouds also produces UV fluorescence (Öberg et al., 2007), and this reemitted energy contribution is thought to be roughly equal to that of direct CR ionization in driving astrochemistry (Moore et al., 2001).

Molecular species such as water, carbon dioxide, methanol, and formaldehyde are produced and form an icy mantle of volatiles around the grains where further irradiation processing occurs. Once mantles have built up, chemistry is not restricted to the surface as reactants can diffuse vertically through the ice layers (Williams and Herbst, 2002). Simulation and laboratory work on irradiation of ices have demonstrated that relatively simple ices can be processed into much more complex molecules such as ethanol, carbonic acid, lactic acid, and long carbon chains (Charnley et al., 2002; Shaw, 2007). Simakov et al. (2002) also showed that nucleotides (the monomer components of DNA and RNA) can be produced by high-energy proton irradiation of simulated interstellar dust grains that contain nucleosides and inorganic phosphate. Although laboratory experiments of ion-irradiated ices have produced many complex organic molecules, the exact nature of the CR flux present in different regions of molecular clouds is not well understood (Moore et al., 2001; Indriolo et al., 2007). Water ice trapped in lunar polar craters and exposed to the CR flux throughout the history of the Solar System offers the possibility of fieldtesting models of astrochemistry and radiation-driven synthesis of organics on icy planetary surfaces and dust grains in interstellar clouds (Lucey, 2000).

As cores within interstellar dust clouds collapse to start the process of star formation, a revolving accretion disk of gas and dust forms around the central protostar. Such a protoplanetary disk is much denser and warmer than the surrounding molecular cloud, and further chemical processing occurs during this stage (see van Dishoeck and Blake, 2003). Organic molecules produced in this circumstellar re- gion become incorporated into comets and meteorites, forming a pool of prebiotic compounds that can be delivered to habitable planets and moons and hence help initiate an origin of life (Ehrenfreund and Charnley, 2000).

Molecular species in interstellar and circumstellar regions have been identified by both their rotational transitions (radiofrequency spectroscopy) and vibrational transitions (IR spectroscopy), and to date over 140 different species have been confirmed (Snow and Bierbaum, 2008). Prebiotic molecules such as amino acids and sugars are expected from theoretical modeling and laboratory experiments but are difficult to detect due to their low abundance in the gas phase (Herbst and van Dishoeck, 2009, presented an excellent review of complex organic interstellar molecules). The simplest sugar, glycolaldehyde, has been independently detected both toward the galactic core (Hollis et al., 2000) and in hot star-forming regions (Beltrán et al., 2009). However, the detection of glycine, the simplest amino acid, was claimed by Kuan et al. (2003) in several hot star-forming regions, but this interpretation has since been contested by the spectral analysis of Snyder et al. (2005). Nevertheless, the increasing realization over recent decades has been the enormous degree of interstellar chemistry, driven overwhelmingly by $C R$ ionization deep inside molecular clouds in addition to UV photochemistry at later stages, which produces molecules of great importance to biology.

Taken to much more speculative lengths, it has been conjectured that, in addition to ionization-driven organic chemistry within irradiated ices, CR may even allow simple living systems to survive in a solid state. Frozen within cometary ice, such organisms may occasionally derive metabolic energy from the tracks of free radicals created by the propagation of CR particles, metabolizing very slowly at perhaps only a few transformations per millennium (Committee on the Limits of Organic Life in Planetary Systems, 2007).

Cosmic ray ionization and driving of chemistry remains a very important process for planetary atmospheres and the icy surface of comets or moons like Europa, as will be addressed later. For now, I will discuss the dynamic nature and shielding of the flux of CR onto habitable planets and moons.

\section{Influence of Cosmic Rays on Planetary Habitability}

\subsection{Astrosphere}

During collapse of the young stellar object and accretion of planets, asteroids, and comets from the protoplanetary disk, ionization from $\mathrm{CR}$ continues to play a key role in driving astrochemistry in the forming solar system. Once the central star has formed, however, it generates a magnetic field and a fast outflowing stream of charged particles, the stellar wind, which blows a cavity in the ISM. The Sun's heliosphere (or astrosphere for the general case) moderates the entry of GCR into the Solar System as a function of particle energy and charge, and also in a time-varying way due to the 11-year cycles of solar activity and "grand minima" of activity such as the Maunder Minimum (1645-1715). Transient and local reductions, known as Forbush decreases, are caused by the shielding effect of the magnetic field carried by coronal mass ejections (reviewed in Cane, 2000). Scherer et al. (2006) and Vainio et al. (2009) presented good reviews of the variability of the particle radiation environment at Earth. Our 
understanding is rather limited, and model dependent, of what the unmodified spectra of CR in interstellar space actually are, as in situ particle energy measurements are only now becoming available as first Voyager 1 on 16 December 2004 (Stone et al., 2005) and then Voyager 2 on 30 August 2007 (Stone et al., 2008) passed the termination shock of the heliosphere (the position of which fluctuates with the strength of the solar wind; Webber and Intriligator, 2011).

The extent of this astrosphere and thus how much protection from GCR is afforded to habitable planets is determined by the balance between the outward pressure of the stellar wind and the density of the local ISM the star is passing through (for good overviews see studies by Scherer et al., 2002, and Fahr, 2004). Passage of the Solar System through dense interstellar clouds would compress the heliosphere and significantly change the interplanetary environment Earth is exposed to (Scherer et al., 2002). This may have occurred in very recent geological history. Zank and Frisch (1999) modeled the interaction between the heliosphere and interstellar clouds and argued that anomalous peaks in Be-10 concentration discovered in Antarctic ice cores that correspond to 33,000 and 60,000 years ago are caused by enhancements in CR flux arriving at Earth's atmosphere due to reduced heliospheric shielding.

It is predicted that dense interstellar clouds could even compress the heliosphere to less than $1 \mathrm{AU}$, which would expose Earth to the unmodulated flux of GCR as well as infalling interstellar gas and dust. Such an extreme phenomenon has been termed "astrospheric collapse" or "descreening" (Smith and Scalo, 2009). Biological hazards from the loss of heliospheric protection include increased surface UV flux due to stratospheric ozone destruction from increased CR flux (Pavlov et al., 2005a) or interstellar hydrogen gas flowing into the atmosphere (Yeghikyan and Fahr, 2004a, 2004b), and interstellar dust triggering runaway glaciation (Pavlov et al., 2005b). Such deleterious outcomes are expected to far outweigh any likely benefits of diminished SCR flux onto Earth during astrospheric collapse.

Smith and Scalo (2009) explored the parameter space of both ISM density and stellar mass to calculate how often planets in the habitable zone of their star would experience such astrosphere descreening events and so be vulnerable to the biological hazards listed above. They found that the risk of descreening decreases strongly with decreasing stellar mass, due both to the weaker focusing of the interstellar flow by the star's gravity and the fact that the habitable zone is closer for lower-mass stars. Descreening could befall a habitable planet around a Sun-type star up to 10 times per billion years, but those orbiting M-class dwarves are virtually never exposed to the severe consequences of astrospheric collapse (Smith and Scalo, 2009).

The shockwave from a nearby supernova explosion could also potentially overcome the solar wind ram pressure and compress the heliosphere to less than 1 AU. Fields et al. (2008) performed numerical hydrodynamical modeling and calculated that a supernova 20 parsecs (pc) distant can penetrate the Solar System to within a few astronomical units, and that material from an event $10 \mathrm{pc}$ distant can collapse the heliosphere to almost exactly $1 \mathrm{AU}$. The debris from a recent nearby supernova, identified by the isotopic signature of radioactive ${ }^{60} \mathrm{Fe}$, has been found in $\sim 2-3$ million-year-old marine sediments (Knie et al., 1999, 2004), and the event may have caused such a significant compression of the heliosphere (Fields et al., 2008).

\subsection{Planetary magnetosphere}

The astrosphere is the first line of shielding against GCR in a planetary system. Many planets, and even moons (e.g., Ganymede; Kivelson et al., 1996), generate a global dipolar magnetic field, and this planetary magnetosphere further screens the spectrum of CR able to reach the atmosphere or surface.

The rigidity of a charged particle is defined as the ratio between its relativistic momentum and charge, and particles with a higher rigidity are deflected to a lesser extent by a magnetic field. The transmission of CR through a magnetosphere can be quantified by the cut-off rigidity, which is the minimum rigidity particles require to reach a particular position within the magnetosphere from a particular arrival direction (Vainio et al., 2009). The magnetic equator of a planetary surface protected by a global dipolar magnetic field has the highest cut-off rigidity, whereas particles with a much lower minimum rigidity are able to access the polar regions. Unmagnetized bodies present no such obstacle, and the CR flux is independent of latitude.

Grießmeier et al. (2005) analyzed the magnetospheric protection an Earth-like planet is likely to receive against CR within the habitable zone of a low-mass star, $0.5 M_{\odot}$ (on the border between $\mathrm{M}$ class and $\mathrm{K}$ class). Orbiting at only $0.2 \mathrm{AU}$ from its star, such a terrestrial planet would become tidally locked, which would result in a rotation rate $2 \%$ that of Earth and consequently a reduced magnetic moment of the global dipole field. The effects on the magnetosphere of such a planetary system are twofold. First, such a slowly rotating planet will generate a weaker magnetic field than Earth (which also has implications for protection from atmospheric erosion by the solar wind; Khodachenko et al., 2007). Second, the habitable zone around a star less massive than the Sun is much closer, so the Earth-like planet is exposed to a more intense stellar wind ram pressure and thus compression of the planetary magnetosphere. Grießmeier et al. (2005) therefore calculated that surface life on a habitable Earth-like planet around an M-class star would be exposed to a higher flux of GCR, which would also have access to a greater area of the planetary surface. Extending this analysis, Grießmeier et al. (2009) showed that the variation in stellar wind pressure with orbital distance has little influence on the shielding of GCR and that the first effect, tidal locking and reduced magnetic moment, is in fact dominant. They also found that the composition and average density of the planet affects the modeled magnetic moment, with a rocky super-Earth receiving 2-3 times more protection against $\mathrm{CR}>00 \mathrm{MeV}$ than an ocean planet of the same mass.

One effect of a reduced magnetic moment and greater GCR penetration may be more widespread loss of the atmospheric ozone shield, as can only occur in polar regions on Earth where the magnetosphere allows energetic particles into the upper atmosphere (Scalo et al., 2007), although in their modeling work Grenfell et al. (2007) found that GCRdriven atmospheric chemistry only minimally affects ozone and water concentrations (also see Section 7.11 of this paper). Life may face no direct radiological hazard from enhanced GCR flux, as Grießmeier et al. (2005) pointed out that a dense 
atmosphere would absorb CR regardless of magnetospheric deflection, a result that has also been discussed for the case of primordial Mars (Molina-Cuberos et al., 2001; Dartnell et al., 2007b; Schneider and Kasting, 2009).

The local source of CR-energetic particles accelerated by the active star-is, however, likely to be a significant threat to habitable planets orbiting young low-mass stars. For the first billion years or so, M-class stars are very active and produce frequent and intense flares and energetic particle events (Scalo et al., 2007), which could produce a SCR flux orders of magnitude higher than that incident upon Earth (Lammer et al., 2009). Habitable M-star planets would thus be exposed to recurrent pulses of SCR, with likely implications for life in the radiation environment created by energetic particles penetrating to the surface (Grießmeier et al., 2005), as well as erosion of the atmosphere (Khodachenko et al., 2007).

Just as the shielding of GCR provided by the astrosphere is time variable, over timescales of both the activity cycle of stars as well as passage through dense interstellar clouds with galactic orbital motion, the shielding provided by a planetary magnetosphere is not constant. Earth's magnetic field reversals can temporarily affect atmospheric chemistry through increased CR penetration (reviewed by Glassmeier and Vogt, 2010), especially if this occurs coincident with another phenomenon such as a nearby supernova, although that is improbable (Crutzen and Brühl, 1996). The magnetic field intensity also fluctuates over geological timescales. Yamazaki and Oda (2002) found a 100,000-year periodicity in the inclination and intensity of the dipolar field over the past 2.25 million years, with the field intensity peaking at no more than twice that of today. This variability is believed to be modulated by the eccentricity of Earth's orbit and may affect $\mathrm{CR}$ penetration into the atmosphere.

Looking at longer timescales, except for transient periods of reversal, the geomagnetic field strength is thought to have varied in intensity by approximately $\pm 50 \%$ from its current magnetic moment of around $8 \times 10^{22} \mathrm{Am}^{2}$ over the past 160 million years (paleomagnetic records and models were reviewed by Aubert et al., 2010). Tarduno et al. (2010) reported results on the Archean geodynamo field strength recorded by magnetic inclusions in single silicate crystals from the Kaapvaal craton in South Africa. They found that 3.4-3.45 billion years ago the geomagnetic field was around $50-70 \%$ that of the present day. This slightly weaker field in the Archean, combined with a greater solar wind ram pressure from a younger, more active Sun, suggests a more compressed magnetosphere. Tarduno et al. (2010) calculated that the magnetopause stand-off distance at that time would have been less than about five Earth radii, which is similar to the effect of a large coronal mass ejection today.

\subsection{Interaction of cosmic rays with atmosphere}

The atmosphere of a planetary body (including large satellites such as Titan) presents the final level of shielding of the surface from ionizing CR with sufficient energy to overcome both the astrosphere and planetary magnetosphere. Although the energy delivered to Earth's atmosphere by GCR is tiny compared to solar electromagnetic radiation, which is greater by a factor of $10^{8}$, they are the only source of ionization at altitudes between 3 and $35 \mathrm{~km}$ (Stozhkov, 2003) and so are a crucial driver of atmospheric chemistry and meteorology. Absorption of this energy flux results in a vertical profile of excitation and ionization of atomic and molecular species within the atmosphere.

An ionosphere is thus created around a planet or moon by the ionization of its neutral atmosphere. In many situations, photoionization by solar UV photons is the dominant source of atmospheric ions, but energetic particle radiation also plays a major role (notably for the nightside ionosphere and bodies at a greater distance from the Sun). Ionization can be caused by charged particles trapped in a planet's dipolar magnetic field precipitating into the upper atmosphere around the poles (e.g., Earth; reviewed by Lyons, 1997), or a moon if it is embedded in its parent planetary magnetosphere (e.g., Titan), as well as GCR and SEP cosmic rays arriving from beyond the magnetosphere (Kivelson and Russell, 1995; also see a recent review of solar system ionospheres by Witasse et al., 2008). Atmospheric ionization is very dynamic and variable over both space and time, being influenced in the short term and longer term by solar UV output and flares; CR variability; gamma-ray, X-ray, and energetic particle influx from supernovae in the stellar neighborhood; and passage of the Solar System through dense molecular clouds (Vasilyev et al., 2008).

Ionization induced by $\mathrm{CR}$ penetrates much deeper into the atmosphere than that from solar UV photoionization; so planetary bodies such as Earth, Venus, and Titan exhibit a second ionosphere below that created by solar UV, which corresponds to the Pfotzer maximum of secondary cascade intensity. For example, the upper ionosphere of Titan, created by solar UV and Saturn's trapped particle belt, has a daytime peak electron density of $3000-6000 \mathrm{~cm}^{-3}$ (depending on solar zenith angle and impact of magnetospheric electrons) at an altitude of around $1000 \mathrm{~km}$, as indicated by modeling studies (Ip, 1990; Roboz and Nagy, 1994; Keller et al., 1998; Banaszkiewicz et al., 2000; Cravens et al., 2004; Wilson and Atreya, 2004; Krasnopolsky, 2009) and corroborated by Voyager 1 radio occultation observations (Bird et al., 1997) and Cassini measurements (Wahlund et al., 2005). The lower, GCR-generated ionosphere has a slightly lower peak electron density of $1000-2000 \mathrm{~cm}^{-3}$ at an altitude of $70-90 \mathrm{~km}$ (modeled by Capone et al., 1980; Borucki et al., 1987, 2006; Molina-Cuberos et al., 1999a, 1999b).

This lower ionization peak from CR is the dominant source of ions in the terrestrial troposphere and drives atmospheric chemistry and potentially weather and climate dynamics. Several recent papers have modeled the ionization profile through Earth's atmosphere from SCR and GCR (for example, see Jackman et al., 2000; Usoskin and Kovaltsov, 2006; Vasilyev et al., 2008), with up-to-date overviews of the field by Bazilevskaya et al. (2008) and Usoskin et al. (2009), these authors comparing the results of several different numerical models.

As well as a potential role of atmospheric cascades in triggering lightning on Earth (Gurevich et al., 1999; Dwyer, 2005), CR ionization has been implicated in driving variability in global climate and the production of organic molecules in the primordial atmosphere, which will be explored in the next two subsections.

\subsection{Cosmic rays and climate forcing}

There exists a long-running debate on whether variations in the CR flux incident on Earth can affect global climate, 
principally by driving cloud formation. The effect of cloud cover is complicated by the fact that it depends on the type; an increase of low-altitude optically thick clouds will result in a cooling through greater albedo and reflection of solar radiation, whereas an increase of high altitude optically thin clouds will warm the planet by trapping reradiated IR. The claimed link between CR flux and climate is that increased ionization of the troposphere by a greater flux of GCR entering the inner Solar System, such as during a period of reduced solar activity, generates additional nucleation sites for water condensation. This increased abundance of nucleation sites causes an increase in the formation of low-altitude clouds and so drives global cooling. For reviews of this hypothesis, see studies by Svensmark (2000, 2007), Carslaw et al. (2002), and Kirkby (2007). However, the actual physical and chemical mechanisms by which $\mathrm{CR}$ can influence condensation nuclei and thus cloud cover are themselves unclear (as reviewed by Carslaw et al., 2002; Kirkby, 2007; Bailer-Jones, 2009). So while an observed correlation between CR intensity and average cloud cover over Earth during the last solar cycle has been reported, critics either question the validity of the correlation (for example, Laut, 2003 presented a cautionary analysis of several claimed correlations between solar activity and climate change) or accept that the correlation is real but explain it by other periodic influences (reviewed by Carslaw et al., 2002). For example, in their simulations of the "ion-aerosol clear-air" hypothesis, Pierce and Adams (2009) found that changes in cloud condensation nuclei from variation in $C R$ over the course of the 11-year solar cycle are 2 orders of magnitude too small to account for the observed changes in low-level cloud cover, but they said that a connection between CR and clouds could operate by other mechanisms. However, looking at shorter timescales, Svensmark et al. (2009) reported a clear link between CR variability during Forbush decreases and measurements of both atmospheric aerosol abundance and low clouds. For further discussion on the potential link between the solar activity-modulated CR flux at Earth, tropospheric ionization, cloud formation, and climate see studies by Svensmark and Friis-Christensen (1997), Bazilevskaya et al. (2000), Carslaw et al. (2002), Shaviv (2005), Section 15 of Scherer et al. (2006), Kirkby (2007), and Bailer-Jones (2009).

Searching for correlations between cooler periods of Earth's climate and inferred increases in the CR environment over geological history, such as during traversal of the arms of the Milky Way, is an even more controversial area as it involves a great number of modeling assumptions and inferences from inconclusive data. For example, Shaviv (2002 and expanded in 2003) modeled the enhancement in GCR flux during the passage of the Solar System through the spiral arms of the Galaxy and claimed a correlation between the calculated timings of these traversals and ice-age epochs in terrestrial history. However, Rahmstorf et al. (2004) questioned the validity of the method used to reconstruct past $C R$ fluxes and their periodicity based on CR exposure dating of iron meteorites, and Overholt et al. (2009) used new data on the structure of the Galaxy and found that any correlation between the 140-million-year terrestrial climate cycle and spiral arm transits disappears. The point is that, although it is not inconceivable that Earth's varying astrophysical environment could drive periodicities in the planet's climate or biosphere, rigorous demonstration of correlations over long time frames, and often necessarily with the use of proxies, has proven exceedingly difficult.

In any case, there exist plausible mechanisms by which changes in CR intensity could drive changes in global climate, so environmental conditions and thus planetary habitability may be influenced by the ionizing radiation environment. The forcing of atmospheric chemistry and global climate by CR ionization of the atmosphere is likely to be particularly significant for planets orbiting stars less massive than the Sun, due to the closer stellar habitable zone and tidal locking (Grenfell et al., 2007; Grießmeier et al., 2009), and especially for planets around the subset of M-class dwarves that exhibit frequent flares (Smith et al., 2004b).

\subsection{Cosmic ray production of organics within the atmosphere}

It is clear that excitation and ionization from CR has a major influence on pushing the atmospheric chemistry of planetary bodies. This energy source generates complex organic species in interstellar molecular clouds, and it is also expected to produce biologically relevant precursor molecules in situ on habitable worlds, including primordial Earth and the complex carbon-nitrogen chemistry and tholin production in Titan's atmosphere (discussed in Section 7.7).

Kobayashi and Tsuji (1997) used high-energy protons to irradiate a gas mixture of carbon monoxide, nitrogen, and water, which is believed to be characteristic of the primordial terrestrial atmosphere, and observed the production of uracil, one of the bases of RNA. In subsequent experiments (Kobayashi et al., 1998, 1999), a similar atmospheric composition was irradiated with high-energy electrons, protons, and helium nuclei, which produced a wide range of amino acids.

Beyond Earth, atmospheric ionization processes are important for other potentially habitable planets and moons in our Solar System, as well as exoplanets and exomoons. Only two potential astrobiological habitats in the Solar System have substantial atmospheres, Venus and Titan, which are discussed next. Studies in which the various effects of extreme atmospheric ionization on the terrestrial environment were analyzed, including ozone destruction and climate forcing, are treated in Section 8.

\subsection{Venus atmosphere ionization}

Venus possesses an atmosphere more dense than that of Earth, but due to the lack of a global magnetic field, CR particles can penetrate further and create an ionization profile deeper into the venusian atmosphere (Upadhyay et al., 1994). Ionizing solar UV does not penetrate much deeper into the venusian atmosphere than $\sim 120 \mathrm{~km}$ altitude, particle radiation from solar flares dominate in the $60-100 \mathrm{~km}$ altitude range, and below $60 \mathrm{~km} \mathrm{GCR}$ are the primary source of ionization (Borucki et al., 1982). This CR-induced ionosphere has been modeled by Dubach et al. (1974), Borucki et al. (1982), Upadhyay et al. (1994), Upadhyay and Singh (1995), and Martin-Torres and Molina-Cuberos (2002).

The total shielding thickness of the venusian atmosphere, around $10^{5} \mathrm{~g} / \mathrm{cm}^{2}$ (Borucki et al., 1982), is two orders of magnitude greater than the terrestrial shield of $1033 \mathrm{~g} / \mathrm{cm}^{2}$ (United States Committee on Extension to the Standard Atmosphere NOAA, 1976), so even the most energetic CR 
present no biological hazard on the surface. The environmental regime of the current venusian surface, however, is not suitable for organic life, and it is the lower or middle global cloud deck that has been proposed as a potential atmospheric habitable zone (e.g., Cockell, 1999; SchulzeMakuch et al., 2004). Between 48 and $57 \mathrm{~km}$ altitude, local physical conditions are conducive to life, with atmospheric pressure between 0.1 and 1 bar and temperature within the range $0-60^{\circ} \mathrm{C}$. In this region, water is available in long-lasting cloud droplets-although of very low $\mathrm{pH}$ from high concentrations of sulfuric acid-and the remaining solar UV (less than a few percent of the incident flux penetrates to the lower cloud level; Cockell, 1999) may be effectively screened or, perhaps, photosynthetically harvested by microorganisms encased in $S_{8}$ (Schulze-Makuch et al., 2004).

Within this putative aerial biosphere, a further survival hazard which may prove to be significant is the radiation environment generated by CR. The peak in CR ionization occurs at an altitude of $25-65 \mathrm{~km}$ in the venusian atmosphere (Upadhyay et al., 1994; Upadhyay and Singh, 1995), which overlaps with the potential habitable zone, as shown in Fig. 6.

The peak particle intensity in Earth's atmosphere, the Pfotzer maximum, occurs at $15-26 \mathrm{~km}$ altitude, depending on latitude and solar activity level (Bazilevskaya et al., 2008). The Concorde used to fly at a cruise altitude of $18 \mathrm{~km}$, well within this radiation peak, and civil aviation still operates typically at $10-12 \mathrm{~km}$ altitude. The total radiation dose from GCR received by a member of the aircrew working a reasonable workload of 700 flight hours per year on subsonic aircraft, including polar routes, is considerably lower than the occupational limit recommended for nonpregnant adults by the International Commission on Radiological Protection (ICRP, 1991) of $20 \mathrm{mSv}$ /year averaged over 5 years (O'Brien et al., 1996). However, the more conservative recommendation for a pregnant woman of no more than $2 \mathrm{mSv}$ over the pregnancy (ICRP, 1991), and not greater than $0.5 \mathrm{mSv}$ in any month (NCRP, 1993), can be exceeded during a standard flight schedule on high-latitude routes (O'Brien et al., 1996; Shea and Smart, 2000). Passengers and crew aboard Concorde were exposed to an equivalent dose rate from GCR 512 times higher than subsonic, lower-altitude, short-haul flights (reviewed recently in Bagshaw, 2008). Every Concorde was fitted with a radiation monitor, and the pilot was alerted to descend to a lower altitude if the dose rate exceeded safety limits during a solar particle event (for details, see Lim, 2002). It is possible that the radiation environment in the Pfotzer maximum of the venusian atmosphere may also represent a survival threat to life already stressed by other environmental hazards such as acidity and solar UV. The radiation hazard would be particularly acute during a large solar flare, especially considering Venus orbits 30\% closer to the Sun than Earth. This potential radiation stress to a venusian aerial ecosystem has not been fully considered before and may prove significant with further study.

\subsection{Titan atmosphere ionization}

The dense atmosphere of Titan (total shielding depth of $11,000 \mathrm{~g} / \mathrm{cm}^{2}$; integral of standard atmosphere density

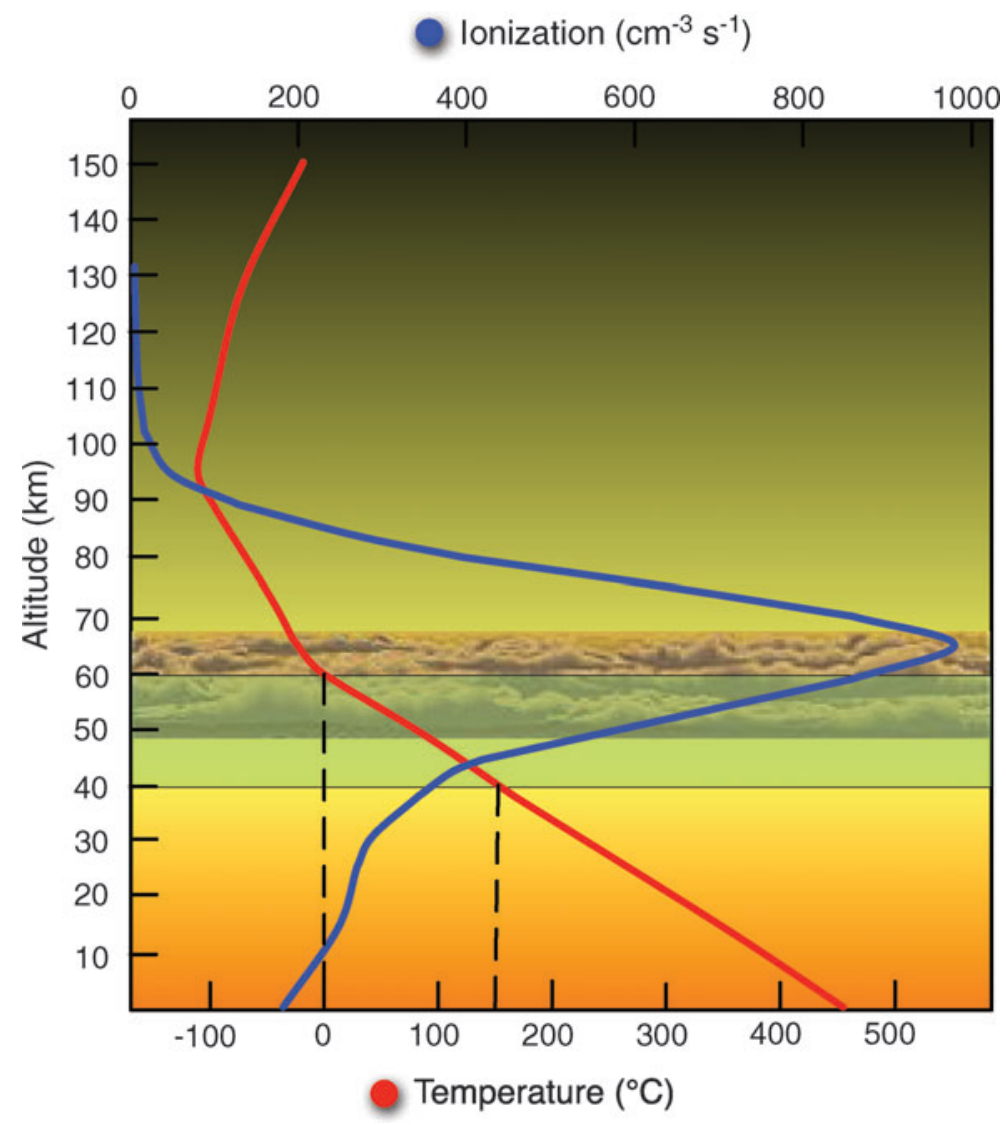

FIG. 6. Schematic of the venusian atmospheric profile, which displays the upper and lower bounds of the three venusian cloud decks and the extent of the aerial habitable zone (green), defined by the region between $0^{\circ} \mathrm{C}$ and $150^{\circ} \mathrm{C}$ (dashed lines projected from red temperature plot). A venusian biosphere could reside in the overlap region between the cloud layer and habitable temperature regime but lies near the peak in atmospheric ionization from cosmic radiation (blue). Diagram constructed using temperature profile from Prinn and Fegley (1987), CR ionization modeling from Upadhyay et al. (1994), and habitable zone discussion in Cockell (1999). Color images available online at www.liebertonline .com/ast 
profile in Yelle et al., 1997, and stated as such by Krasnopolsky, 2009) is believed to have a similar chemistry to Earth's primordial atmosphere (Molina-Cuberos et al., 1999b). This atmospheric chemistry is driven at high altitudes by ionization from solar UV and trapped particle radiation in the magnetosphere of Saturn, creating an ionosphere at an altitude above about $800 \mathrm{~km}$ (MolinaCuberos et al., 1999a; Cravens et al., 2006), the composition of which has now been measured (Cravens et al., 2006; Coates et al., 2007) by the Cassini orbiter (reviewed recently by Brown et al., 2009). Solar UV irradiance in the outer Solar System is much reduced by the inverse square law, and Titan's atmosphere, with its composition of nitrogen, methane, and hydrocarbons, is very opaque to UV. Saturn's magnetic field at Titan is not powerful enough to shield CR flux significantly, and Titan generates no intrinsic magnetic field of its own (Backes et al., 2005), so the titanian atmosphere is exposed to a significant CR flux that penetrates far deeper into the atmosphere than UV photons. Cosmic rays thus create a second, lower ionosphere in Titan around 50-100 km altitude, which is comparable in intensity to the higher zone created by solar UV and trapped electrons. This CR-induced ionization of the Titan atmosphere has been modeled by several authors (Capone et al., 1980; Borucki et al., 1987, 2006; Molina-Cuberos et al., 1999a, 1999b), but of greatest significance in the interests of the astrobiology of Titan is the role this ionization-driven atmospheric chemistry plays in creating organic molecules.

The upper, solar UV-generated ionosphere has been observed (by instruments aboard Cassini) to contain both positively charged organic molecules (Cravens et al., 2006) and heavy negative ions (Coates et al., 2007; Vuitton et al, 2009), which are believed to be important in the atmospheric hydrocarbon chemistry and transformation of simple gaseous species into organic-rich aerosol compounds-the socalled tholins. These long-chain hydrocarbons form the atmospheric haze layers and fall to deliver organics to the surface of Titan (Waite et al., 2007). Although these orbital detections of large species are from the upper ionosphere, similar organics are also expected to be produced in the lower, CR-generated ionosphere, and so contribute to the organic inventory and biological potential of Titan's surface environment. Cosmic rays that penetrate through the atmosphere may also induce additional organic synthesis on the surface, especially in the polar lakes of methane and ethane (Raulin et al., 2009), such as driving the polymerization of HCN (Raulin et al., 1995).

While the terrestrial surface biosphere is largely sheltered from CR by the geomagnetic field and shielding atmospheric column, the surface of two planetary bodies with astrobiological potential (Mars and Europa), and meteorites potentially bearing microbial life ejected from planets receive no such protection. Indeed, the unshielded flux of CR onto the martian surface is one of the major environmental hazards to remnant surface life, even if present as spores or cryopreserved in permafrost.

\subsection{Sterilization of Mars' surface}

Extensive geomorphological (Squyres and Kasting, 1994) and ground-based mineralogical and geochemical (e.g., Squyres et al., 2004) evidence indicates the ancient existence of flowing and standing water on the martian surface: a warmer, wetter Mars. The current martian surface environment is characterized by low temperatures, low atmospheric pressure, and consequent low availability of liquid water, and it is exceedingly hostile to the persistence of life (Horneck, 2000). The thin atmospheric layer offers only minimal shielding against solar UV (Patel et al., 2002), which would rapidly kill any exposed microorganisms (Schuerger et al., 2006) and photolyze organic molecules such as amino acids (ten Kate et al., 2005). This long-term UV flux is also expected to have produced a wind-dispersed layer of chemical oxidants on the surface (Zent and McKay, 1994; Yen et al., 2000).

Another major consequence of the thin atmospheric column and the absence of a martian dipole magnetic field (although it is believed to have once generated a global field; Acuña et al., 1999) is the poor shielding of Mars against CR. Energetic GCR and SEP are essentially unimpeded onto the martian surface, and the cascades of secondary particles they create penetrate several meters underground. Thus, the penetration of cosmic radiation probably exceeds the depth of oxidizing conditions in most parts of the martian surface and is a major threat to the survival of life held dormant by the freezing dry conditions.

A major research effort has been focused on modeling the martian surface ionizing radiation environment for the implications to human health during future astronaut exploration (e.g., Simonsen et al., 1990, 1991; Simonsen and Nealy, 1993; Kim et al., 1998; Cucinotta et al., 2002; De Angelis et al., 2004; Saganti et al., 2004). More specifically in the interests of astrobiology, several modeling studies have involved calculation of the radiation dose profile generated by $\mathrm{CR}$ through the martian regolith to predict the likely survival times of spore-forming or radiation-resistant bacterial strains such as D. radiodurans in the subsurface (Mileikowsky et al., 2000; Pavlov et al., 2002; Kminek et al., 2003; Dartnell et al., 2007a, 2007b, 2010). While there are uncertainties in both the physics of high-energy particle transport and the biological response to irradiation, these studies broadly agree and have found that the top $20 \mathrm{~cm}$ of the martian surface is sterilized of even the most radiation-resistant microorganisms (based on terrestrial models such as $D$. radiodurans) within a million years or so, with greater survival found at increasing depths due to shielding, especially in ice deposits (Dartnell et al., 2007a). In material with a density of around $1 \mathrm{~g} / \mathrm{cm}^{3}$, the more energetic GCR cascades begin to dominate over the average SEP flux within around $10 \mathrm{~cm}$ depth (Dartnell et al., 2007a), and radiation from the GCR cascades peaks at (the Pfotzer maximum) $25-50 \mathrm{~cm}$ deep and at a third of that depth within solid rock or regolith (Mileikowsky et al., 2000; Pavlov et al., 2002; Dartnell et al., $2007 \mathrm{~b}$ ). Beneath the penetration of GCR cascades, at 3-4 m depth, depending on surface properties, the remaining source of ionizing radiation is the decay of radionuclides in the surrounding regolith. This natural radioactivity of martian regolith is believed to be much lower than that of terrestrial rocks (Mileikowsky et al., 2000) but would still sterilize deep dormant bacteria in 40 million years (Pavlov et al., 2002; Dartnell et al., 2007a).

The ancient martian surface, with a temperature and pressure regime conducive to liquid water, however, would have received significant CR shielding from a thicker atmospheric column (Molina-Cuberos et al., 2001; Dartnell et al., 
2007b; Schneider and Kasting, 2009), so life could have originated under more favorable conditions.

While the flux of cosmic ionizing radiation onto the surface of Mars is likely to pose a considerable hazard to the survival of microbial life in the near subsurface, the situation may be reversed for Jupiter's icy moon Europa, and ionization from particle radiation may actually help support a subsurface biosphere.

\subsection{Support of europan biosphere}

Europa is thought to possess a global ocean of liquid water beneath an outer ice shell of perhaps only $\sim 1 \mathrm{~km}$ thickness (Greenberg et al., 2000), although other interpretations of surface features imply a much thicker (tens of kilometers) ice layer (Head et al., 1999). Sunlight cannot penetrate kilometers of ice, so a europan biosphere would not have access to widespread photosynthesis (Reynolds et al., 1983; Chyba and Hand, 2001). There has been some discussion, however, as to whether geothermal blackbody light emitted by seafloor hot water plumes (emission peaking at IR wavelengths) could support photosynthetic life, alongside chemoautotrophic organisms that harness inorganic chemical energy, around black smokers on Earth and, by extension, possibly Europa (e.g., White et al., 2002; Beatty et al., 2005; Rathgeber et al., 2008).

An alternative energy source for a chemosynthetic-based ecosystem could be provided by the charged particle radiation trapped within Jupiter's magnetosphere (see Fig. 1), the jovian equivalent of Earth's Van Allen belts (Chyba and Phillips, 2001). Particle radiation delivers more energy relevant to ice chemistry to the europan surface than solar UV and is comparable to internal heat flux from radiogenic or tidal heating (Cooper et al., 2001).

Irradiation of the europan surface water ice would produce oxidants, such as oxygen and hydrogen peroxide, and radiolysis of carbon dioxide in the ice and subsequent freeradical chemistry is expected to also generate reduced organic molecules such as formaldehyde (Delitsky and Lane, 1998; Chyba and Phillips, 2001). Such a radiolytically generated disequilibrium of oxidized and reduced chemical species could power a chemoautotrophic-based biosphere if these redox couples can be washed into the europan ocean, as discussed further below.

Niches of chemoautotrophs have been discovered on Earth that survive on radiogenic products. Lin et al. (2006) described a microbial community that survived at $2.8 \mathrm{~km}$ depth in basaltic crust, independent of photosynthesis or its products for tens of millions of years, by metabolizing sulfate with hydrogen released by radiolysis of the fracture water, driven by radionuclide decay in the surrounding basalt. There has even been a report of melanin-containing fungi, isolated from high-radiation environments like Chernobyl or the cooling water of nuclear reactors, that exhibit faster growth under conditions of high ionizing radiation flux than without it (Dadachova et al., 2007). The suggestion is that melanin enables the cells to extract energy from the ionizing radiation to drive their metabolism, a process termed "radiosynthesis." A similar, weakly supported claim has been made for growth of photosynthetic bacteria and algae in the dark, supported by a low flux of gamma radiation (Luckey, 2008). If these preliminary studies on metabolism directly powered by ionizing radiation were to gather further corroboration, they would represent very interesting results indeed.

Thus, terrestrial chemoautotrophic organisms that are powered by nuclear decay energy are known, and a europan biosphere could be similarly maintained by redox couples generated by the ionizing flux of trapped particle radiation onto the surface ice. A radiogenically supported europan biosphere is only feasible, however, if these redox couples can be transported below to the ocean at high enough rate.

In an initial modeling effort, Chyba (2000a, 2000b) calculated that the rate of radiogenic nutrients imported into the ocean could support only a severely limited biomass. An updated model that incorporates new estimates of the depths of radiation processing and impact gardening of the europan surface ice has given a more optimistic conclusion (Chyba and Phillips, 2001). However, even assuming 100\% conversion efficiency of this redox energy into biomass, microbial cell densities would still be very low and may frustrate attempts at biosignature detection.

While surface irradiation could maintain a microbial ecosystem, Chyba and Phillips (2001) deemed it unlikely that oxygen levels could rise high enough to support macrofauna akin to the aquatic animal life of our own oceans. On the other hand, Irwin and Schulze-Makuch (2003) drew attention to terrestrial invertebrates and vertebrates that can survive cold anoxic conditions and argued that metazoan life at the top of a europan food chain could perhaps approach the size of a tadpole or brine shrimp. More recently, Greenberg (2010) argued that various resurfacing mechanisms on Europa ensure the radiation-oxidized ice layer is at least $300 \mathrm{~m}$ thick, so the ocean could be oxygenated enough to support even a large macrofaunal ecosystem, of the order of 3 million tons of fish.

Another astrobiological consideration of CR is that the cellular damage they wreak may also pose an upper limit on the survival time of microorganisms embedded within meteorites and so restrict panspermia and the natural transfer of life between planetary bodies.

\subsection{Panspermia}

The theory of (litho)panspermia, that life can be transferred between planetary bodies within meteorites, has matured over recent years (see up-to-date reviews by Burchell, 2004, and Nicholson, 2009) with the results of simulations of planetary ejection by low-angle impact (Nyquist, 1983) or spallation (Melosh, 1984), orbital transfer dynamics calculations (Gladman et al., 2005), and experimental work on microbial survival of the associated shock pressures and temperatures (Fajardo-Cavazos et al., 2009), and resistance to the space environment (Horneck et al., 1994, 2001). On the whole, such experiments find that non-negligible proportions of microbial populations could indeed survive the shock of ejection or reentry and if protected from solar UV can tolerate the vacuum and desiccation of the space environment for protracted periods, thus supporting the conjecture of biological transfer within the Solar System. The possibility has also been discussed that Earth reseeded itself with returning ejecta after sterilizing impacts during the Late Heavy Bombardment (Wells et al., 2003).

During the transit between planetary bodies, microorganisms contained in the ejected rock fragments would be unprotected by planetary magnetic field or atmosphere, so 
they would be exposed to the SEP and GCR flux, shielded from the charged particle bombardment only by the surrounding meteorite. Several studies have modeled the propagation of energetic CR particles through meteoritic rock to estimate microbial survival times. Mileikowsky et al. (2000) found that with meteorites smaller than about $60 \mathrm{~cm}$ in diameter the dose rate experienced in the center is actually increased by the shielding rock, due to the generation of secondary radiation cascades. For microorganisms sheltered in the center of a boulder at least $2.6 \mathrm{~m}$ in diameter, the shielding against external CR would be effective, and the radiation hazard from decay of radionuclides in the surrounding rock would begin to dominate. Using similar methods, Mileikowsky et al. (2000) and Clark et al. (1999) calculated that at $1 \mathrm{~m}$ depth in ejected regolith a sterilizing dose is accumulated on the order of millions of years. Mileikowsky et al. (2000) concluded that, within the distribution of likely meteorite sizes and transit times, the transfer of viable cells from Mars to Earth must be considered not only possible but highly probable. In the case of the martian meteorites so far recovered, however, Clark (2001) calculated that, due to their small size and period of interplanetary transit, all would have been exposed to strongly sterilizing doses of CR.

Interstellar panspermia, on the other hand, does not seem likely, considering the low probabilities of ejection from one planetary system and capture by another, the unlikelihood of safe landing on a planetary body at interstellar transfer velocity (Melosh, 2004), and the high accumulated levels of CR damage during the long transit. Valtonen et al. (2009) concluded that no potentially life-bearing ejecta from another solar system landed on Earth before life had already arisen on the planet, unless this transfer occurred between sister planetary systems in the same star cluster shortly after formation. Indeed, the accumulated background irradiation from GCR has been proposed as a natural mechanism for sterilizing (in the interests of planetary protection) any interstellar probes we launch, particularly to investigate exoplanetary systems (Cockell, 2008).

\subsection{Degradation of biosignatures}

As well as posing a survival threat to microbes in the martian surface or within meteorites, ionization and nuclear reactions driven by $C R$ could degrade biosignatures indicating the prior existence of life (that may now have fallen extinct). Also, atmospheric ionization in a planet orbiting in the habitable zone of a M-class red dwarf star may act to mask the atmospheric disequilibrium indicative of a biosphere.

Considering Mars first, both experimental irradiation and radiation modeling studies have looked at the likely rate of erasure of different detectable biosignatures in the near subsurface. These include the destruction rate of amino acids (Kminek and Bada, 2006), racemization of an amino acid enantiomeric excess (Bonner, 2000, and references therein), alteration of biogenic organics (Court et al., 2006), nuclear reactions that lead to the loss of ${ }^{13} \mathrm{C} /{ }^{12} \mathrm{C}$ isotopic bias (Pavlov et al., 2002), and biopolymer fragmentation (Pavlov et al., 2002). The general issue is that after millennia of cosmic irradiation it may be difficult to distinguish between breakdown debris of unambiguous biomolecules, a valid signature of extinct life, and simple abiotic organic species created in situ by prebiotic chemistry or exogenously delivered by meteoritic or cometary infall. There may only be a limited window of opportunity to find evidence of extinct surface life before it is degraded below the detection limit. Pavlov et al. (2002) estimated that in the martian near subsurface complete radiolytic macromolecular breakdown occurs within $10^{8}$ to $10^{9}$ years and eradication of a biogenic ${ }^{13} \mathrm{C} /{ }^{12} \mathrm{C}$ isotopic bias in less than 25 million years. Kminek and Bada (2006) calculated that, to detect the remnant amino acids from Hesperian life, which occurred about 3 billion years ago, it would be necessary to drill to a depth of $1.5-2 \mathrm{~m}$. However, the dependence of biomolecule destruction on radiation dose, particle linear energy transfer (see, e.g., Nelson, 2003), and temperature is not well understood (see discussion by Dartnell et al., 2007b), so there is uncertainty in these estimates.

Furthermore, the biosignature detection instrumentation itself may be vulnerable to ionization by $\mathrm{CR}$, particularly proposed designs that employ organic dyes or antibodies, and this possibility has been explored recently. Thompson et al. (2006) ran proton and alpha irradiations on fluorescent dyes proposed for use in labeled assays, and Le Postollec et al. (2009a, 2009b) conducted both modeling and experimental neutron irradiation work on antibodies and dyes. These studies found that the antibody function or fluorescence response was not significantly affected by ionizing radiation fluences comparable to those expected during a martian mission.

Cosmic rays may also play a significant role in masking biosignatures in the atmospheres of extrasolar planets. Biosignatures could potentially be remotely detected on exoplanets by IR spectroscopy and identification of atmospheric chemistry far from thermodynamic equilibrium, such as the co-presence of oxygen (or its photolytic product, ozone) and methane (Lovelock, 1965; Sagan et al., 1993; Des Marais et al., 2002; Kaltenegger et al., 2007). Planets orbiting within the habitable zone of M-class stars are expected to possess weaker magnetospheric shields and so would be exposed to higher fluxes of GCR than Earth, as discussed above in Section 7.2. Grenfell et al. (2007) considered the likely effects of this more intense GCR environment in producing nitrogen oxides and driving atmospheric chemistry to modify potential biomarker molecules such as ozone, nitrous oxide, and methane, all of which can reach much higher concentrations in the atmosphere of M-class star planets and make particularly conspicuous biosignatures (Segura et al., 2005). They found that the profile of ozone in the planetary atmosphere is only modestly affected by GCR-induced nitrogen oxide production, while nitrous oxide and methane show negligible difference. Thus, this is not anticipated to hinder spectroscopic detection of biosignatures on planets in the habitable zone of M-class stars. However, as with Grießmeier et al. (2005), Grenfell et al. (2007) did not consider the SEP flux from the host star itself, which may constitute a nonnegligible atmospheric ionization source. Segura et al. (2010), whose work will be discussed further in Section 8.3, did model the effects of SEP flux from a particularly large flare on an active $\mathrm{M}$ dwarf hitting an Earth-like planet that lacked a magnetic field in the habitable zone. They concluded that, although atmospheric ozone may be severely depleted, methane concentration is not perturbed, and so a single large 
flare would not hinder spectroscopic atmospheric biosignature detection. The effects of repeated flares before the atmosphere can return to equilibrium, however, are not well understood.

At the beginning of this section, I reviewed how the background flux of CR onto Earth, or any similar planet shielded by magnetic field and thick atmosphere, poses no significant threat to life on the surface. However, extreme astrophysical events can release an intense flux of CR or energetic electromagnetic radiation and, thus, present a sporadic but acute ionizing radiation hazard to a planetary biosphere, as is discussed in detail in the next section.

\section{Extreme Radiation Events}

While a planetary magnetic field or significant atmospheric column can effectively shield the surface of a habitable world from the radiological effects of the background CR flux, even an Earth-like planet could be seriously affected by a more intense pulse of ionizing radiation. The consequences of an extremely energetic particle event, with a high enough fluence and average particle energy to penetrate even dense atmospheric shields and thus present a direct radiation hazard on the surface, should not be neglected.

Dar et al. (1998) discussed the effects of a high-energy CR jet that may also be generated at the same time as a GRB (Vietri et al., 2003). Dar et al. (1998) argued that such a highly collimated relativistic particle jet would have a beaming angle $\Delta \Omega \leq 0.01$, similar to those observed or estimated for active galactic nuclei and microquasars, and would reach a distance of around $1 \mathrm{kpc}$ before becoming disrupted. If such a jet were to strike a terrestrial planet, the particle flux would not only be extremely high but the average particle energy would be a thousand times higher than the background CR. The energy deposition in the atmosphere over days to months would equal that of the normal background GCR over 10 million years. A large fraction of the energy of the jet primary particles, with a typical energy of $\sim 1 \mathrm{TeV} /$ nucleon, would be transformed into energetic muons by extensive air showers. These are highly penetrating and would irradiate with lethal doses to depths of hundreds of meters of water or rock. In addition to the ionizing muon flux and stratospheric ozone depletion, the particle jet would create radioactive isotopes in the atmosphere and surface by nuclear spallation, which would be dispersed globally by winds. Dar et al. (1998) estimated that such a CR jet might be targeted toward Earth by a nearby neutron star merger on the order of every 100 million years and so could account for some of the five metazoan mass extinctions of the past 570 million years.

Even if energetic CR do not penetrate to a planetary surface to pose a direct radiological hazard, there are significant consequences of the extreme atmospheric ionization caused by violent astrophysical events like supernovae or GRB. The potential outcomes of extreme ionization events are summarized in the schematic diagram in Fig. 7, with the labels relating to the main text section numbers below.

\subsection{Supernova destruction of the ozone shield}

The danger presented to a biosphere by intense photon or particle radiation from a source such as a supernova, even if no significant amount of the ionizing radiation directly reaches the surface, was recognized by Ruderman (1974). Ionizing radiation propagating through the atmosphere causes the dissociation of $\mathrm{N}_{2}$ to produce $\mathrm{NO}_{x}$ compounds (most crucially $\mathrm{NO}$ and $\mathrm{NO}_{2}$ ), which act catalytically to destroy stratospheric $\mathrm{O}_{3}$. The ozone layer shielding Earth's surface from biologically active bands of solar UV radiation is only $3-4 \mathrm{~mm}$ thick at standard temperature and pressure, and Ruderman (1974) estimated that this shield would be substantially depleted for up to a century by a supernova at 50 light-year range. UV is harmful to both DNA and photosynthetic pigments (Horneck, 1995), and an increase in flux of only $10-20 \%$ can be lethal to many terrestrial organisms such as phytoplankton, the base of many food chains (Thomas and Melott, 2006). Aikin et al. (1980) argued that stratospheric ozone would actually be enhanced at first by the intense UV flash of a nearby supernova, before the gamma-ray flux overtakes Earth several months later and destroys the ozone again.

In subsequent years, many authors have attempted to quantify the degree of stratospheric ozone depletion and time before recovery, and likely effects to the biosphere, from a nearby supernova explosion (e.g., Ruderman, 1974; Whitten et al., 1976; Reid et al., 1978; Ellis and Schramm, 1995; Crutzen and Brühl, 1996; Gehrels et al., 2003; Thomas and Honeyman, 2008). The modeled results often disagree; therefore, the conclusions drawn disagree as well with regard to the extent of biological perturbations on terrestrial planets from nearby supernovae. Recently, for example, Gehrels et al. (2003) considered ozone depletion from both the gamma-ray photons and later-arriving pulse of CR from a supernova in a two-dimensional atmospheric model. They calculated that, for the surface biologically active UV flux to be doubled, the supernova must occur within 8 pc of Earth, an event which happens only every 1.5 billion years; thus Gehrels et al. (2003) concluded that supernovae are less important than other potential causes of mass extinction. In general, impacts on Earth's biosphere from nearby supernovae are estimated to occur on the timescale of every few hundred million to several billion years; thus they do pose a plausible hazard to life. There is no evidence, however, for a direct link between supernovae and a past mass extinction.

Thomas et al. (2008) considered the likely effects on Earth of a specific massive star, $\eta$ Carinae, that appears ready to detonate. At $2.3 \mathrm{kpc}$ distance, $\eta$ Carinae is unlikely to pose any serious hazard as a normal supernova, but it may produce a superluminous supernova that could have potential implications even at such a great range. However, Thomas et al. considered atmospheric ionization (and subsequent ozone depletion), enhanced CR flux, and endocrine disruption from intense blue light, and concluded that even if superluminous, $\eta$ Carinae would be unlikely to endanger the terrestrial biosphere.

\subsection{Gamma-ray bursts}

Gamma-ray bursts are far more energetic than supernovae and have been identified as a potential threat to biospheres by Thorsett (1995). Thorsett (1995) estimated that GRB, which are potentially caused by the merging of binary neutron stars, occur in our own Galaxy about every $10^{5}$ to $10^{6}$ years, with a burst occurring uncomfortably close (within $1 \mathrm{kpc}$ ) perhaps every $10^{8}$ years (see also Melott et al., 2004, 


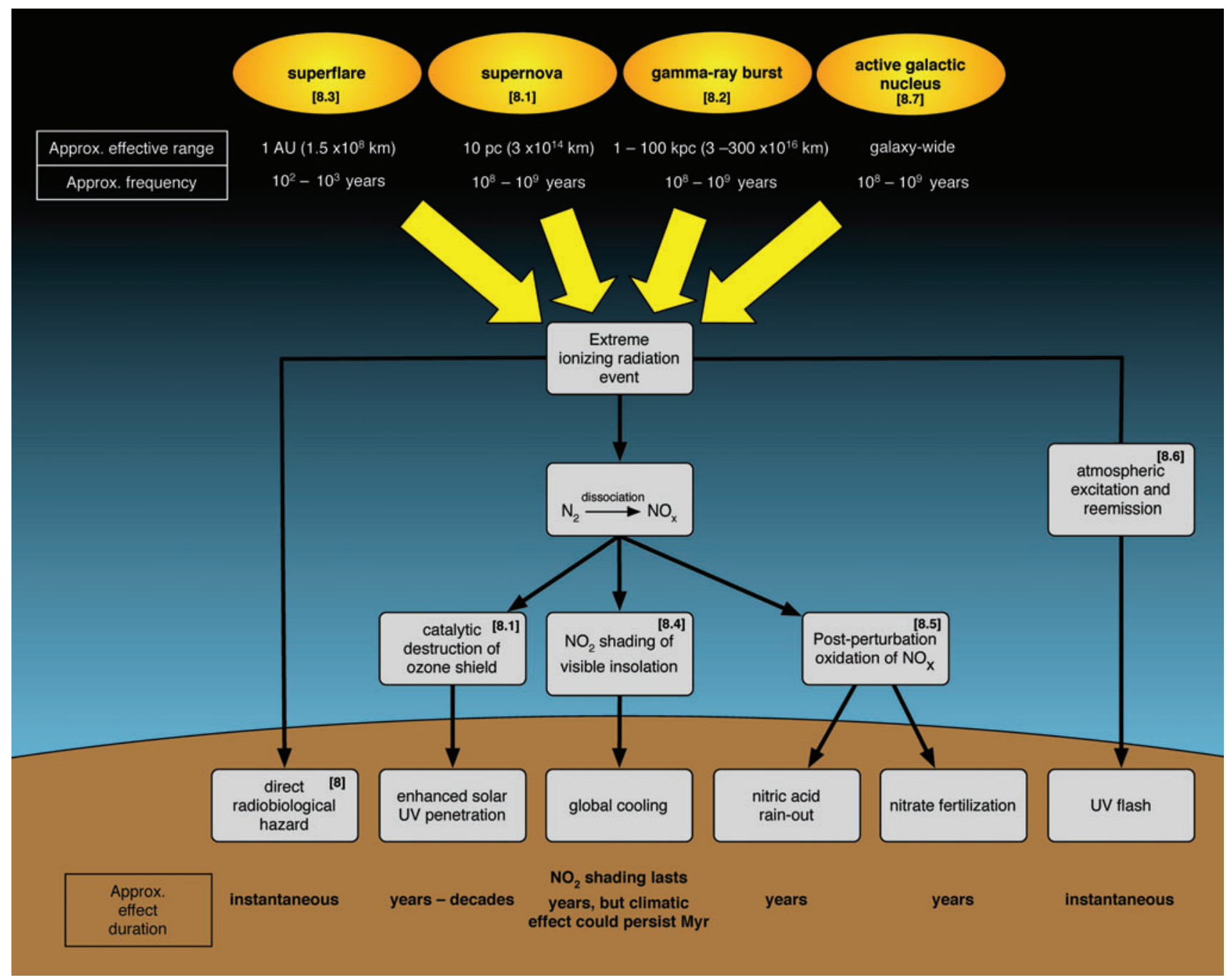

FIG. 7. Schematic summarizing the cascade of effects and hazards to the biosphere of extreme ionizing radiation events imposed on a habitable planet by sporadic astrophysical sources. The labels refer to subsection numbers in Section 8 of the main text. Color images available online at www.liebertonline.com/ast

and Dermer and Holmes, 2005, for more recent estimates of the frequency of GRB per galaxy). Thorsett (1995) calculated that a GRB as distant as the galactic center, almost $8 \mathrm{kpc}$ remote, would have similar consequences to the nearby supernova considered by Ruderman (1974). It has also been suggested that the occurrence of GRB could periodically sterilize the Galaxy of land-based life (Annis, 1999).

The effects of supernovae or GRB on a planetary atmosphere or biosphere would be similar, although from very different effective ranges (see Fig. 7). Considering both their relative intrinsic intensity and galactic frequency, the greatest danger to a biosphere is posed by supernovae less than $10 \mathrm{pc}$ distant (Reid et al., 1978; Ellis and Schramm, 1995; Gehrels et al., 2003) and directed GRB 1-100 kpc remote (Scalo and Wheeler, 2002; Melott et al., 2004, 2005; Dermer and Holmes, 2005; Thomas and Melott 2006; Martín et al., 2009).

Aside from intrinsic luminosity, the major difference between GRB and supernova events is the time course over which the pulse of energetic photons occurs. Ejzak et al. (2007) studied how the atmospheric end point of extreme ionization events from GRB or supernovae is affected by the blast duration of ionizing photons (from $10^{-1}$ to $10^{8} \mathrm{~s}$ ). They found that the ultimate atmospheric effects do not vary significantly, so the effects of extreme atmospheric ionization from different astrophysical events can be satisfactorily approximated by disregarding the time development of such events and considering only the total radiation fluence.

Analyzing the effects of a GRB within $2 \mathrm{kpc}$ of Earth, Thomas et al. (2005a) calculated that the irradiation would globally deplete stratospheric ozone by $35 \%$, an event that is statistically estimated to have occurred at least once in the last billion years. Ozone recovery from such destruction would take at least 5 years, during which time the surface would be exposed to up to a 3-fold increase in solar UVB flux, which the authors predict would trigger widespread extinction.

The threat posed to terrestrial planetary atmospheres and biospheres by GRB has been reviewed recently by Thomas and Melott (2006). They noted that, although polar regions experience greatest long-term ozone depletion and 
consequent enhanced UVB flux, DNA damage is limited here by reduced incident solar irradiance, so the increase in UVB flux is most hazardous to land-based or shallow water-borne life (less than $10 \mathrm{~m}$ depth) in midlatitudes.

Galante and Horvath $(2007 \mathrm{a}, 2007 \mathrm{~b})$ split the impact of a GRB-directed beam into four distinct phenomena: direct gamma-ray transmission to the planetary surface, the UV flash created by reemission in the atmosphere (an effect first considered by Smith et al., 2004a; see Section 8.6 of this current review), ozone depletion, and increased flux of CR. They found that the first three can affect a biosphere from huge distances, greater than the diameter of the Galaxy, whereas the enhanced CR flux would only be lethal for a very close GRB, only a few parsecs away.

\subsection{Superflares}

Extreme ionization of the atmosphere could also be caused by a source much more local than GRB or supernovae: an anomalously energetic solar particle event. Reid et al. (1976) considered the likely terrestrial effects of solar flares 10 and 100 times more intense than the August 1972 event, widely accepted to be the largest solar particle event of the space age (Mewaldt, 2006). They calculated a global ozone depletion of around $25 \%$ and $50 \%$, respectively, and consequent increase in biologically effective UV flux at the surface by a factor of 1.5 and 2.5 , but only if such a flare coincidentally occurred within the period of a few thousand years during a polarity reversal of the geomagnetic field. Even without the co-occurrence of an intense solar particle event, Reid et al. (1976) calculated stratospheric ozone levels would drop by $4.3 \%$ during polarity reversal due to increased penetration of GCR.

Thomas et al. (2007) modeled the specific case of the 1859 "Carrington event" solar flare, which is believed to be one of the most powerful white-light flares ever observed. Nitrate deposits in polar ice indicate that the Carrington flare was the largest solar proton event of the last 450 years, with a $>30 \mathrm{MeV}$ solar proton fluence around 4 times greater than the measured August 1972 event (Shea et al., 2006). Thomas et al. (2007) calculated that the intense proton flux from such a flare would have caused up to $14 \%$ localized ozone depletion for about 4 years and an increase in nitrate deposition such that it would agree reasonably with measurements taken from ice cores. On the other hand, Jackman et al. (2000) modeled the perturbation to terrestrial stratospheric chemistry and ozone destruction due to two large solar particle events from the past 30 years and modeled the August 1972 and October 1989 events as well. They estimated that the chemical state of the atmosphere, such as stratospheric chlorine levels, can actually cause an increase in total ozone for a few years after such an event.

The hazard from flares may be particularly acute for Earth-like planets orbiting within the habitable zone of active M-class red dwarf stars. Segura et al. (2010) modeled the atmospheric effects of the UV and SEP flux from a large flare hitting an Earth-like planet that lacked a magnetic field in the habitable zone of the active M dwarf AD Leonis. They found that the UV emission from the flare had negligible impact on atmospheric ozone but that the nitrogen oxides produced by ionization from the energetic protons resulted in an ozone depletion of more than $90 \%$ within two years of the flare, which took almost 50 years to recover. However, even at maximum ozone depletion, the surface UV dose rate for DNA damage was only $4 \%$ larger than that on Earth.

Alongside the destruction of the ozone shield, several other potentially catastrophic insults to a planetary biosphere from an acute atmospheric ionization event have been identified, which are reviewed in Subsections 8.4-8.6 below.

\subsection{Global cooling}

Beyond ozone destruction, the elevated levels of nitrogen dioxide generated in the atmosphere by cosmic radiation from a superflare, supernova, or GRB are also expected to cause other effects on planetary habitability. Nitrogen dioxide is a brown gas that absorbs strongly at visible wavelengths, so a spike in atmospheric $\mathrm{NO}_{2}$ levels could significantly reduce solar insolation that reaches the ground and could potentially trigger global cooling.

Stratospheric nitrogen dioxide produced by the superflare modeled by Reid et al. (1976) was calculated to decrease surface visible solar irradiance by several percent, but due to the heat reservoir of the oceans such a transient perturbation to the global thermal budget may not trigger any lasting climatic change (Reid et al., 1978). For a nearby supernova, Reid et al. (1978) postulated that the resultant cooling of Earth's surface (estimated at up to a $3 \mathrm{~K}$ decrease in global average temperature) from nitrogen dioxide opacity may in fact cause a more pronounced biological impact than the ozone destruction and increased surface UV flux. Such a global cooling event would reduce atmospheric water vapor content by about $20 \%$, which would result in further cooling through reduced efficacy of the greenhouse effect and widespread drought conditions. Melott et al. (2005) found that, averaged globally, atmospheric $\mathrm{NO}_{2}$ produced by a GRB would reduce surface solar irradiance by $1 \%$, but up to $30 \%$ reduction in polar regions (due to poleward transport of GRB products within the stratosphere), for several years. Melott et al. (2005) speculated that such an input could possibly trigger a global cooling event, although Thomas reported that no detailed simulations of the likely climatic response have yet been run (Thomas, 2009).

Tanaka (2006) considered another potential mechanism for triggering global cooling by a nearby supernova: greater CR flux and ionization of the lower atmosphere. These atmospheric ions would act as condensation nuclei and thus increase cloud formation at low altitude, possibly triggering a major ice-age epoch.

A further complication of the likely climatic consequences is that nitrogen dioxide shading and cooling of the lower atmosphere and surface is not equivalent to a simple decrease in solar irradiance, as this absorbed energy leads to heating of the stratosphere. A hotter stratosphere with increased thermal emission to the ground would partially compensate for visible shading, which would reduce surface cooling by up to a factor of 2 (Reid et al., 1978; Melott et al., 2005) but also affect the dynamic interaction between the stratosphere and troposphere with possible consequences for global climate.

\subsection{Nitric acid rain-out}

As the atmosphere returns to its pre-perturbation composition, nitrogen oxides are removed by oxidation and 
rain-out as dilute nitric acid $\left(\mathrm{HNO}_{3}\right)$. This would increase the acidity of global precipitation for several years, and a nearby GRB could substantially lower the $\mathrm{pH}$ of surface waters and adversely affect ecosystems. Thomas and Honeyman (2008) looked specifically at the consequences of a GRB on amphibian populations, as previous studies have found that a combination of higher UVB flux, elevated nitrate concentrations, and lower $\mathrm{pH}$ had a synergistic deleterious effect on such organisms. They considered a worst-case scenario of a GRB hitting the North Pole at autumnal equinox (as in Thomas et al., 2005b). Although the gamma photon fluence was short (10s), the subsequent rain-out of $\mathrm{HNO}_{3}$ from the atmosphere was calculated to last several years. However, the increase in nitrate concentration in surface water was calculated to be insufficient to give a significant negative impact on amphibian populations.

Another effect of a major atmospheric ionization event from cosmic radiation may be potentially beneficial to life. The productivity of both terrestrial and marine ecosystems is often limited by the availability of nitrates (Vitousek and Howarth, 1991; Elser et al., 2007). The increase in nitrate concentrations produced by rain-out of cosmogenic nitrogen oxides following an extreme atmospheric ionization event may therefore aid primary producers in ecosystems by providing accessible nitrogen and acting as fertilizer (Melott et al., 2005; Thomas et al., 2005a, 2005b). Thomas et al. (2005a) calculated that a GRB $2 \mathrm{kpc}$ away would generate around $0.5 \mathrm{~g} / \mathrm{m}^{2}$ of nitrates deposited globally. Thomas et al. (2005a) hypothesized that such a GRB irradiation event at the end of the Ordovician period (440 million years ago), although difficult to verify, may have promoted the colonization of land by nitrate deposition, as well as elevated atmospheric $\mathrm{NO}_{2}$ levels, triggering a brief glaciation by blocking a small percentage of solar radiation. Melott et al. (2004) and Melott and Thomas (2009) argued the case for a GRB cause of the late Ordovician mass extinction.

Such radiogenic nitrates may become preserved in sediments and ice deposits and serve as evidence for geologically recent supernovae near Earth. Brakenridge (1981) calculated the impact times on Earth of the gamma and X-ray radiation flash from observed nearby supernova remnants and highlighted the close Vela remnant, 500 pc from Earth. Brakenridge (1981) reported the discovery of fixed nitrogen deposits around the world that date to the predicted occurrence of the Vela supernova, around 11,000 years ago, and argued that this supernova may have been the trigger for a cooling event at the same time, the Younger Dryas (Berger, 1990; Alley, 2000).

Thomas (2009) presented a brief summary of the likely effects of a GRB within a few kiloparsecs on Earth's atmosphere and biosphere, including all three of the above threats: ozone depletion, nitrogen dioxide shading, and nitric oxide rain-out. Thomas reported on simulations that covered a range of gamma-ray fluences from the burst, hitting the Earth at various latitudes at both equinoxes and solstices (to account for seasonal variations in atmospheric chemistry) and at different times of day. The largest impact on the biosphere was found to be due to ozone depletion and enhanced UVB irradiance. Weighting the calculated solar spectral irradiance by using biological effectiveness functions (for effects such as DNA damage), Thomas found that annual UV-induced DNA damage would be up to 16 times greater than the global pre-burst average.
Thus, even a short-duration burst of energetic cosmic radiation can have effects on atmospheric chemistry and, consequently, the biosphere, that are long-lived and global in their impact.

\subsection{Ultraviolet flash}

In addition to the above delayed threats from extreme atmospheric ionization, shielding of an intense CR pulse by a planetary atmosphere can have other deleterious effects to a surface biosphere, only recently described. Over the past few years, Smith, Scalo, and Wheeler have co-authored a series of papers detailing their investigations into the transformation of the incident cosmic ionizing radiation by the shielding atmosphere into biologically hazardous bands of UV light, through a process similar to aurora: electronic excitation of atmospheric molecules and reemission of photons.

Smith et al. (2004a) considered the flux of cosmic X-rays and gamma rays from sources such as solar flares, supernovae, and GRB, incident onto habitable exoplanets with varying atmospheres. Even thin atmospheres (although still dense enough to maintain habitable conditions on the surface) will block incident $X$-rays, but still transmit a significant fraction of the gamma-ray flux to the surface. Exoplanets with atmospheres thicker than about $100 \mathrm{~g} / \mathrm{cm}^{2}$ would be effectively shielded against the penetration of both X-rays and gamma rays, but interactions within the atmosphere would reprocess almost all the incident photon energy into diffuse UV emission. These longer wavelengths are more effectively transmitted through the atmosphere (although dependent on atmospheric thickness and UV absorbers), and for a planet with an atmosphere of around $100 \mathrm{~g} / \mathrm{cm}^{2}$ up to $10 \%$ of the incident energy could reach the planetary surface as UV reemission from the atmosphere, which would subject exposed surface organisms to a harsh UV insult.

Smith et al. (2004b) expanded on this analysis for Archean Earth (3.9-2.5Ga), considering the photon spectrum from nearby supernovae as well as solar flares. Assuming only Rayleigh scattering of UV photons by the $\sim 1000 \mathrm{~g} / \mathrm{cm}^{2}$ atmosphere and no UV filters such as $\mathrm{O}_{3}$, Smith et al. (2004b) found that up to $4 \%$ of the incident ionizing radiation reaches the surface as biologically effective UV radiation (200$320 \mathrm{~nm}$; UVC and UVB bands). For the current terrestrial situation, including an ozone shield, only $0.2 \%$ of the incident energy reaches the surface as biologically active UV reemission, although this is still many orders of magnitude higher than the energy of the ionizing radiation that penetrates to the surface. Considering just the solar flare influence on planets orbiting within the habitable zone of a Sun-type star, significant fluxes of hazardous UV would occasionally penetrate to the surface, even on evolved terrestrial planets with a photosynthetic biosphere and ozone shield, but would not exceed the direct UV from the sun. For terrestrial planets orbiting within the habitable zone of lower-mass stars (<0.5 solar mass), such as M-class dwarves, however, the energy from solar flare $\mathrm{X}$-rays and gamma rays redistributed into short wavelength UV by the atmospheric shield would completely dominate the direct UV flux from the parent star (Smith et al., 2004b). Organisms evolving on such a planetary surface may therefore experience frequent pulses of elevated UV flux and, if not adequately protected, consequent episodes of cellular damage or hypermutation. 
Scalo and Wheeler (2002) attempted to calculate the frequency with which terrestrial planets are exposed to an ionizing radiation flux from a GRB in the Galaxy sufficient to cause a direct biological effect through DNA damage. About 100-500 such potentially biologically significant events or "jolts" are predicted to occur every billion years for eukaryotic-like organisms and around 100 times fewer for prokaryotic life, which is more radiation resistant, through direct ionizing radiation beneath thin atmospheres (such as modern-day Mars) or through UV reemission by thick atmospheres (such as Earth). However, due to the short duration of GRB (only one hemisphere of a planet would be exposed) and the effective shielding of organisms provided by only $50 \mathrm{~cm}$ of water or shallower depths for rock, only a tiny proportion of these stochastic irradiation events might actually significantly perturb the biosphere.

Martín et al. (2009) also considered the UV flash generated by atmospheric reemission from a GRB at different epochs in the evolution of Earth's biosphere and atmosphere, and they reported that a surface ecosystem during the Mid-Proterozoic would likely be most stressed. Galante and Horvath (2007a, b) presented a more comprehensive analysis of GRB impact on an Earth-like atmosphere and biosphere, modeling the effect of direct gamma-ray transmission, UV flash, stratospheric ozone destruction, and enhanced CR flux. They found that global environmental and biological upsets may be caused by GRB sources up to $100 \mathrm{kpc}$ remote. Peñate et al. (2010) found that the UV reemission from a GRB $2 \mathrm{kpc}$ away could penetrate up to $75 \mathrm{~m}$ through clear water (20-30 $\mathrm{m}$ for more turbid water) to severely affect aquatic photosynthetic primary producers, particularly if a night flash, to a great depth, and thus also affect the ecosystems depending upon them.

In any case, it has become clear that the pulse of ionizing radiation from a supernova or GRB can affect the planetary surface UV environment (beneath an atmosphere) in both the short and long term. Atmospheric irradiation and reemission produce a transient burst of UV, while the perturbed atmospheric chemistry and nitric oxide generation leads to destruction of an ozone shield and increased transmission of solar UV. The rest of this section deals with variations in the GCR flux incident on a habitable planet.

\subsection{Active galactic nuclei}

Another astrophysical source of energetic ionizing radiation, alongside supernovae, GRB, and superflares, which is not so often discussed, is the accelerated particle emission from the active nucleus of a galaxy. The energy source driving active galactic nuclei is believed to be a supermassive black hole and the surrounding accretion disc; such objects are very luminous in X-rays. If the core of the Milky Way has been active in this way in its past, or in other galaxies during a phase of nucleus activity, there would also be an enhanced particle radiation flux that is likely to be a more significant hazard to habitable systems in the galaxy than the X-ray flux. Clarke (1981) considered a simple model of the situation and found that the particle radiation flux at Earth would be enhanced by a factor of around 100 during the lifetime of galactic nucleus activity. Such an increased ionizing particle flux would be concomitant with planetary hazards such as ozone depletion and enhanced surface radiation environment as described above.

\subsection{Variation in the galactic cosmic ray flux}

Beyond possible activity of the core of the Milky Way, other mechanisms may cause variation in the GCR flux incident on the Solar System. This variability of the incident GCR flux is independent of the modulation of the GCR flux through the Solar System by the varying heliosphere (see Section 7.1, as well as reviews by Scherer et al., 2006, and Vainio et al., 2009). Although diffusion of charged particles along interstellar magnetic field lines acts to produce an essentially isotropic and uniform background flux of CR throughout the Galaxy, moderate statistical variations are expected due to the random space-time distribution of supernovae (Erlykin and Wolfendale, 2001). In particular, Svensmark (2006) modeled the CR flux into Earth's atmosphere over the history of the planet based on estimations of the galactic star formation (and thus also supernova) rate, SEP flux, and diminishing solar activity modulation of GCR, and reported that particle fluxes were at most twice their current intensity over the past 4.5 billion years. The distribution of supernovae throughout the Galaxy and, thus, the expected frequency of acute cosmic ionizing radiation events impacting a habitable planet is also a critical factor in defining the extent of the galactic habitable zone and its spatial evolution over time (Lineweaver et al., 2004).

Medvedev and Melott (2007) argued for a causal link between a claimed $62 \pm 3$ million-year cycle in Phanerozoic animal fossil diversity (discovered by Rohde and Muller, 2005, and confirmed to be a robust signal by Lieberman and Melott, 2007) and periodic variation in the GCR flux. They argued that, due to the vertical oscillation of the Solar System through the plane of the Galaxy (with a period of 64 million years) and an anisotropy in the extra-GCR flux caused by the motion of the Milky Way through the intergalactic medium, there is a cyclical variation in the CR impinging on Earth over the right timescale to explain the fossil diversity variation. Although Medvedev and Melott (2007) proffered no mechanism for actually linking extra-GCR flux and terrestrial biodiversity, Melott et al. (2008) investigated one plausible mechanism: CR-induced destruction of ozone and increased surface exposure to solar UVB. Even at the upper end of the range of plausible parameters explored, Melott et al. (2008) found only a $2 \%$ reduction in global ozone levels, no more than currently being caused by anthropogenic pushing of atmospheric chemistry (such as chlorofluorocarbon release) and far less than that predicted for a nearby GRB. So even though the duration of increased atmospheric ionization and consequent ozone depletion from enhanced extra-GCR is predicted to last around a million times longer than that from a GRB, it is unlikely to exert a great biological stress unless coincidentally occurring in combination with other threats to the biosphere.

In addition to variation in the background GCR flux, the atmospheres and biospheres of planetary bodies are exposed to extreme stochastic events such as the superflares, nearby supernovae, and remote GRB discussed above. Thus, the cosmic ionizing radiation environment of a potentially habitable planet or moon is highly dynamic, with temporal variability across a great range of timescales:

- minutes for the most intense stage of GRB,

- hours to days for solar particle events, 
- days to weeks for the initial electromagnetic pulse from supernovae,

- thousands of years if the solar system passes through the expanding shock cavity of a nearby supernova remnant or the heliosphere is compressed by passage through dense interstellar clouds,

- tens of millions of years from oscillations of the Solar System about the galactic plane.

Variations in the GCR flux, or indeed stochastic pulses of extreme ionizing radiation flux from nearby supernovae or GRB, may be recorded by spallation products and cosmogenic isotopes embedded in the lunar regolith and then shielded and protected by a later lava flow (Crawford et al., 2010; Fagents et al., 2010). Measurement of the CR bombardment history of unprotected planetary surfaces such as meteorites and lunar rocks was reviewed by Reedy et al. (1983) and Eugster (2003).

So far in this review, I have considered the effects of exogenous cosmic radiation incident onto a planetary atmosphere or surface. Potentially habitable planets and moons are also likely to include a significant component of radiogenic isotopes and so generate their own intrinsic ionizing radiation environment, the astrobiological implications of which are explored in the following section.

\section{Intrinsic Planetary Radiation Environment}

The decay of radioisotopes within a planetary body serves as a heat source (along with remnant heat from formation) for volcanism and plate tectonics, both of which are thought to have been important in maintaining habitable conditions on Earth for long periods (e.g., see Lammer et al., 2009). In addition to this role in habitability, the intrinsic radiation environment of a planet or moon, generated by radioisotope decay as opposed to $\mathrm{CR}$ impinging onto an atmosphere and biosphere, is also a critical factor in habitability and the origin of life. On Earth, ambient radiation levels from radionuclide decay peaked about four billion years ago, at a level approximately 7 times higher than today, and have been steadily decreasing since (Karam and Leslie, 1999). However, the rise in free oxygen over geological time and its enhancing effect on DNA damage through increasing reactive oxygen species generated by radiolysis may have served to steady mutation rates over the history of life (Karam et al., 2001). The role of enhanced primordial radioactivity in driving prebiotic chemistry on Earth is reviewed below.

\subsection{Radiation and prebiotic chemistry}

The radiolytically driven production of biologically relevant molecules in primordial Earth's oceans, from decay of potassium-40, was considered by Draganic (2005). While the annual energy available from dissolved potassium- 40 was much less than that from other sources, it is argued to be significant, as it was distributed evenly throughout the primordial ocean, including the deep ocean where other energy sources such as solar UV would have been absent. It is estimated that $10^{14} \mathrm{~g}$ of organic compounds would have been created over 100 million years by in situ radiation in the primordial oceans (Draganic, 2005).

Primordial carbonate minerals, co-deposited with radionuclide metal cations such as potassium-40, rubidium-87, and the uranium-235, uranium-238, and thorium-232 radioactive decay families, would have self-irradiated and may have generated substantial quantities of organic precursor species (Collins et al., 2000). Production of organics by carbonate irradiation has been achieved experimentally (Kolomnikov et al., 1982; Albarrán et al., 1987), and on early Earth these precursors could have entered the hydrosphere and contributed to the origin of life by direct dissolution or weathering out of the carbonate rocks. On the other hand, synthesis of organic molecules on clay mineral substrates may have been hampered by potassium- 40 radiolytic destruction exceeding the rate of free-radical-induced synthesis or polymerization (Draganic, 2000). There may have also been significant synthesis of prebiotic molecules at kilometers depth in the Archean terrestrial crust from radionuclide irradiation of water held in the porous rock (Garzón and Garzón, 2001).

It has been proposed that the observed enantiomer bias in terrestrial biology, the exclusive use of L-amino acids and Rsugars, was fostered on primordial Earth by the role of radionuclide decay in prebiotic chemistry (see Fitz et al., 2007, for a review of theories proposed to explain the origin of biological homochirality). For example, the polarization of electrons released by beta decay of radionuclides has been suggested as a causative agent in selecting the enantiomer bias by preferential synthesis or degradation of either enantiomer in an initially racemic mixture. However, systematic reviews of the varied experimental approaches attempted to demonstrate such an effect have concluded that there is no substantiating evidence for such a causal connection between nuclear parity violation and selection of an enantiomer bias in biomolecules (reviewed in Bonner, 2000, and Fitz et al., 2007). A more recent alternative hypothesis posits that supernovae in the vicinity of the ISM from which the Solar System condensed were responsible for the generation of the observed bias toward L-amino acids in carbonaceous chondrite meteorites. Boyd et al. (2010) argued that amino acid enantiomers become aligned in opposite directions along intense magnetic field lines and consequently R-amino acids are preferentially destroyed by the pulse of neutrino radiation from a nearby supernova. The mechanism proposed by Boyd et al. (2010) is that atoms of ${ }^{14} \mathrm{~N}$ would become aligned along the intense magnetic field lines of the nascent neutron star in a manner dependent on the electronic structure and, thus, chirality of the parent molecule; hence they would also force the orientation of amino acids or their precursors that contain the ${ }^{14} \mathrm{~N}$ atom. The subsequent pulse of electron antineutrinos from the supernova would preferentially destroy one spin orientation of the ${ }^{14} \mathrm{~N}$ and thus the chiral molecule containing it, which would produce an enantiomeric bias in the surviving molecules in the ISM out to a significant range from the supernova. Later chemical amplification of this enantiomer bias, and incorporation of the astrochemistry-produced organics into carbonaceous chondrites and delivery to the surface of primordial Earth, could explain the observed L-amino acid preference in the terrestrial biosphere.

Parnell (2004a) considered a subsequent role for radioactivity of mineral sands in the origin of life, as an energy source that drives the polymerization and precipitation of complex organic molecules from precursors. Methane outgassed from an impact crater through hydrothermal systems 
containing radioactive minerals could also have been an important site for organic synthesis on early Earth or perhaps Mars (Parnell et al., 2006). Indeed, carbonaceous coatings are often observed as having accreted around uranium- or thorium-rich mineral grains of Archean sedimentary rocks. Court et al. (2006) experimentally observed ionizing radiation from uranium- and thorium-rich grains polymerizing simple hydrocarbons such as methane into complex polycyclic aromatic hydrocarbon-rich organic matter. They argued that the prebiotic organic inventory synthesized from such a process, either in situ on early Earth or delivered from irradiated extraterrestrial material, made a significant input to the origin of life.

Such radiolytically driven organic synthesis may even feed a limited biosphere on an orphaned terrestrial planet gravitationally ejected from its solar system into interstellar space (Stevenson, 1999).

\subsection{Natural fission reactors}

An example of an extremely intense intrinsic source of particle radiation on a planetary surface is provided by the ancient natural fission reactors in Oklo, Gabon (see reviews by Zetterström, 2000, and Jensen and Ewing, 2001). Here, multiple small pockets of uranium ore contained within layers of sedimentary rocks achieved criticality around 2 billion years ago. Lens-shaped reactor cores, around $10 \mathrm{~m}$ in diameter and $0.5 \mathrm{~m}$ thick, sustained nuclear fission on and off for $10^{5}$ to $10^{6}$ years (Nagy et al., 1991), with the water table acting as an efficient moderator of neutrons and the fission reaction (Gauthier-Lafaye et al., 1996). The fission reaction created temperatures estimated between $160^{\circ} \mathrm{C}$ and $360^{\circ} \mathrm{C}$ during criticality (Nagy et al., 1991), which drove the circulation of hot fluids and hydrothermally altered the host rock for up to $50 \mathrm{~m}$ from the reactor core, transforming the sandstone strata into clays (Gauthier-Lafaye et al., 1996) and producing abundant solidified bitumen from organic molecules originating in the host rock (Parnell, 1996).

Close to the reactor core, the radiation field would have been a complex mix of fission fragments, energetic electrons, neutrons, and gamma rays (Draganic et al., 1983). It has been estimated by in-pile dosimetry that, operating at a kilowattlevel power, the Oklo natural nuclear reactors would have generated radiation dose rates of over $1 \mathrm{kGy} / \mathrm{h}$ within their cores. However, a more constraining biological hazard would have been the high temperatures created by the fission reaction. With the thermal gradient in the clays surrounding the core calculated to be $100^{\circ} \mathrm{C} / \mathrm{m}$ (GauthierLafaye et al., 1996), microbial life several meters away from the cores may have been able to survive the thermal hazard and been exposed to only the more penetrating neutron and gamma radiation from the fission reactions. Furthermore, $14 \%$ of the fission products have half-lives longer than one year and so would have created an elevated radiation environment for subterranean life during periods of reactor shutdown and also migrated outward from the core into the surrounding environment (Draganic et al., 1983).

The Oklo reactors were active 2 billion years ago, which was too late for an involvement in the origins of life. Indeed, the conditions that favor the formation of concentrated deposits of uranite necessary to achieve criticality are thought to have been created by a redox boundary produced by bi- ology (Gauthier-Lafaye and Weber, 2003). Uranium is soluble under slightly oxic conditions and when reduced it precipitates, usually as uranite. The Oklo reactors were created when the water-soluble $\mathrm{U}^{6+}$ ion, which was oxidized in the groundwater from dissolved oxygen released by early cyanobacteria, migrated underground until it encountered a reduction front created by subsurface organics. The uranium precipitated out of solution along this redox boundary to produce a rich deposit.

Abiotic processes for creating sufficiently enriched deposits of fissile isotopes, which could have played a role in life's origin, have also been discussed. Adam (2007) presented the hypothesis of a possible role in prebiotic chemistry for a Hadean or early Archean heavy mineral placer beach 4.3 billion years ago. A placer beach is formed by gravitational separation of mineral grains by density during the sedimentation process and can concentrate heavy minerals like uranium to 70-90 wt \% at particular sand horizons up the beach. Such an enrichment of heavy mineral grains, including radioactive isotopes of thorium and uranium (which would have been stable in the non-oxidizing primordial atmosphere) as well as other actinide elements, would have favored much prebiotic chemistry. Actinides can act as catalysts for oligomerization; and the energy released by their radioactive decay, and potentially by a self-sustaining fission reaction far earlier than the Oklo-type deposit discussed above, could have driven radiolysis and synthesis of simple organic molecules from inorganic carbon dissolved in the seawater between mineral grains, as Adam also demonstrated experimentally.

If synthesis and concentration of organic molecules by radioactive grains is indeed a necessary process in the origins of life, then this is another way in which plate tectonics may be a prerequisite for the habitability of a terrestrial planet (in addition to maintaining the composition of the atmosphere by recycling volatiles; e.g., see recent review by Lammer et al., 2009). The concentration of radioactive monazite and uranite mineral grains in certain locations is dependent on the erosion of granitic continental crust, so plate tectonics may perform a vital role in driving prebiotic chemistry (Parnell, 2004b).

\section{Conclusions}

This review has surveyed the broad range of current research where ionizing radiation from exogenous $C R$, intense electromagnetic radiation from sporadic astrophysical sources, and intrinsic mineral radioactivity of a world exerts significant influence over processes pertinent to astrobiology. Ionizing radiation has traditionally been considered as mainly detrimental to biology, and ionization and radiolysis are indeed greatly destructive to the complex molecules and polymers of life and thus pose a severe hazard to the development and survival of organisms. So, the ionizing radiation flux may well constitute a limiting factor for the persistence of life on unshielded planetary surfaces such as Mars (or perhaps high in the venusian atmosphere), on worlds orbiting within the habitable zone of stars less massive than the Sun, and during the interplanetary transfer of microorganisms via panspermia. Earth's surface is protected from CR by screening from the Sun's heliosphere, the global magnetosphere, and absorption of particle cascades by the 
atmosphere, and any habitable exoplanet would experience similar multiple layers of shielding. However, even wellprotected Earth-like worlds may receive biosphere-threatening radiation hazards during rare, but extreme, astrophysical events. Cosmic rays may also act to degrade or mask detectable biosignatures in the martian surface or atmosphere of an exoplanet, as well as impairing the functionality of the biodetection instrumentation on probes.

The synthesis presented here, though, has demonstrated the many varied ways in which ionizing radiation is also beneficial and plays a vital role in providing habitable environments and supporting the development of life in the Cosmos. These functions include the synthesis of biogenic elements and the driving of prebiotic chemistry to generate organic molecules in the ISM, circumstellar dust during formation of planetary systems, and the atmospheres, oceans, and subsurfaces of habitable planets and moons. Indeed, in the case of Europa, the flux of trapped ionizing radiation onto the icy surface and radiolytic production of oxidants and organics may even nourish an oceanic ecosystem; a curious counterpoint to the biosphere-limiting effect of $\mathrm{CR}$ in the martian near subsurface.

The interplay between ionizing radiation and life is rich with complexity and conflicting effects, and the topics highlighted here represent many fruitful areas for future research.

\section{Acknowledgments}

The author is supported by UCL Institute for Origins postdoctoral research associateship funding. He thanks Ian Crawford, Mike Storrie-Lombardi, and Serena Viti for many useful discussions, and he thanks two anonymous peer reviewers who by their thorough attention have helped improve the quality of this manuscript considerably.

\section{Author Disclosure Statement}

No competing financial interests exist.

\section{Abbreviations}

$\mathrm{CR}$, cosmic rays; GCR, galactic cosmic rays; GRB, gammaray bursts; ISM, interstellar medium; pc, parsec (parallax of one arcsecond); SCR, solar cosmic rays; SEP, solar energetic particles.

\section{References}

Abel, T., Bryan, G., and Norman, M. (2002) The formation of the first star in the Universe. Science 295:93-98.

Acuña, M.H., Connerney, J., Ness, N., Lin, R., Mitchell, D., Carlson, C., McFadden, J., Anderson, K., Reme, H., Mazelle, C., Vignes, D., Wasilewski, P., and Cloutier, P. (1999) Global distribution of crustal magnetization discovered by the Mars Global Surveyor MAG/ER experiment. Science 284:790-793.

Adam, Z. (2007) Actinides and life's origins. Astrobiology 7: 852-872.

Aikin, A., Chandra, S., and Stecher, T. (1980) Supernovae effects on the terrestrial atmosphere. Planet Space Sci 28:639-644.

Akaboshi, M., Fujii, N., and Navarro-González, R. (2000) The Role of Radiation in the Origin and Evolution of Life, Kyoto University Press, Kyoto.
Albarrán, G., Collins, K., and Collins, C. (1987) Formation of organic products in self-radiolyzed calcium carbonate. $\mathrm{J} \mathrm{Mol}$ Evol 25:12-14.

Allen, A., Li, Z.-Y., and Shu, F.H. (2003) Collapse of magnetized singular isothermal toroids. II. Rotation and magnetic braking. Astrophys J 599:363-379.

Alley, R. (2000) The Younger Dryas cold interval as viewed from central Greenland. Quat Sci Rev 19:213-226.

Amsler, C., Doser, M., Antonelli, M., Asner, D., Babu, K., Baer, H., Band, H., Barnett, R., Bergren, E., Beringer, J., Bernardi, G., Bertl, W., Bichsel, H., Biebel, O., Bloch, P., Blucher, E., Blusk, S., Cahn, R., Carena, M., Caso, C., Ceccucci, A., Chakraborty, D., Chen, M., Chivukula, R., Cowan, G., Dahl, O., D'Ambrosio, G., Damour, T., de Gouvêa, A., DeGrand, T., Dobrescu, B., Drees, M., Edwards, D., Eidelman, S., Elvira, V., Erler, J., Ezhela, V., Feng, J., Fetscher, W., Fields, B., Foster, B., Gaisser, T., Garren, L., Gerber, H., Gerbier, G., Gherghetta, T., Giudice, G., Goodman, M., Grab, C., Gritsan, A., Grivaz, J., Groom, D., Grünewald, M., Gurtu, A., Gutsche, T., Haber, H., Hagiwara, K., Hagmann, C., Hayes, K., Hernández-Rey, J., Hikasa, K., Hinchliffe, I., Höcker, A., Huston, J., Igo-Kemenes, P., Jackson, J., Johnson, K., Junk, T., Karlen, D., Kayser, B., Kirkby, D., Klein, S., Knowles, I., Kolda, C., Kowalewski, R., Kreitz, P., Krusche, B., Kuyanov, Y., Kwon, Y., Lahav, O., Langacker, P., Liddle, A., Ligeti, Z., Lin, C., Liss, T., Littenberg, L., Liu, J., Lugovsky, K., Lugovsky, S., Mahlke, H., Mangano, M., Mannel, T., Manohar, A., Marciano, W., Martin, A., Masoni, A., Milstead, D., Miquel, R., Mönig, K., Murayama, H., Nakamura, K., Narain, M., Nason, P., Navas, S., Nevski, P., Nir, Y., Olive, K., Pape, L., Patrignani, C., Peacock, J., Piepke, A., Punzi, G., Quadt, A., Raby, S., Raffelt, G., Ratcliff, B., Renk, B., Richardson, P., Roesler, S., Rolli, S., Romaniouk, A., Rosenberg, L., Rosner, J., Sachrajda, C., Sakai, Y., Sarkar, S., Sauli, F., Schneider, O., Scott, D., Seligman, W., Shaevitz, M., Sjöstrand, T., Smith, J., Smoot, G., Spanier, S., Spieler, H., Stahl, A., Stanev, T., Stone, S., Sumiyoshi, T., Tanabashi, M., Terning, J., Titov, M., Tkachenko, N., Törnqvist, N., Tovey, D., Trilling, G., Trippe, T., Valencia, G., van Bibber, K., Vincter, M., Vogel, P., Ward, D., Watari, T., Webber, B., Weiglein, G., Wells, J., Whalley, M., Wheeler, A., Wohl, C., Wolfenstein, L., Womersley, J., Woody, C., Workman, R., Yamamoto, A., Yao, W., Zenin, O., Zhang, J., Zhu, R., Zyla, P., Harper, G., Lugovsky, V., and Schaffner, P. (2008a) Cosmic rays. Phys Lett B 667: 254-260.

Amsler, C., Doser, M., Antonelli, M., Asner, D., Babu, K., Baer, H., Band, H., Barnett, R., Bergren, E., Beringer, J., Bernardi, G., Bertl, W., Bichsel, H., Biebel, O., Bloch, P., Blucher, E., Blusk, S., Cahn, R., Carena, M., Caso, C., Ceccucci, A., Chakraborty, D., Chen, M., Chivukula, R., Cowan, G., Dahl, O., D'Ambrosio, G., Damour, T., de Gouvêa, A., DeGrand, T., Dobrescu, B., Drees, M., Edwards, D., Eidelman, S., Elvira, V., Erler, J., Ezhela, V., Feng, J., Fetscher, W., Fields, B., Foster, B., Gaisser, T., Garren, L., Gerber, H., Gerbier, G., Gherghetta, T., Giudice, G., Goodman, M., Grab, C., Gritsan, A., Grivaz, J., Groom, D., Grünewald, M., Gurtu, A., Gutsche, T., Haber, H., Hagiwara, K., Hagmann, C., Hayes, K., Hernández-Rey, J., Hikasa, K., Hinchliffe, I., Höcker, A., Huston, J., Igo-Kemenes, P., Jackson, J., Johnson, K., Junk, T., Karlen, D., Kayser, B., Kirkby, D., Klein, S., Knowles, I., Kolda, C., Kowalewski, R., Kreitz, P., Krusche, B., Kuyanov, Y., Kwon, Y., Lahav, O., Langacker, P., Liddle, A., Ligeti, Z., Lin, C., Liss, T., Littenberg, L., Liu, J., Lugovsky, K., Lugovsky, S., Mahlke, H., Mangano, M., Mannel, T., Manohar, A., Marciano, W., Martin, A., Masoni, A., Milstead, D., Miquel, R., Mönig, K., Murayama, H., 
Nakamura, K., Narain, M., Nason, P., Navas, S., Nevski, P., Nir, Y., Olive, K., Pape, L., Patrignani, C., Peacock, J., Piepke, A., Punzi, G., Quadt, A., Raby, S., Raffelt, G., Ratcliff, B., Renk, B., Richardson, P., Roesler, S., Rolli, S., Romaniouk, A., Rosenberg, L., Rosner, J., Sachrajda, C., Sakai, Y., Sarkar, S., Sauli, F., Schneider, O., Scott, D., Seligman, W., Shaevitz, M., Sjöstrand, T., Smith, J., Smoot, G., Spanier, S., Spieler, H., Stahl, A., Stanev, T., Stone, S., Sumiyoshi, T., Tanabashi, M., Terning, J., Titov, M., Tkachenko, N., Törnqvist, N., Tovey, D., Trilling, G., Trippe, T., Valencia, G., van Bibber, K., Vincter, M., Vogel, P., Ward, D., Watari, T., Webber, B., Weiglein, G., Wells, J., Whalley, M., Wheeler, A., Wohl, C., Wolfenstein, L., Womersley, J., Woody, C., Workman, R., Yamamoto, A., Yao, W., Zenin, O., Zhang, J., Zhu, R., Zyla, P., Harper, G., Lugovsky, V., and Schaffner, P. (2008b) Passage of particles through matter. Phys Lett B 667:267-280.

Annis, J. (1999) An astrophysical explanation for the Great Silence. J Br Interplanet Soc 52:19-25.

Aubert, J., Tarduno, J., and Johnson, C. (2010) Observations and models of the long-term evolution of Earth's magnetic field. Space Sci Rev 155:337-370.

Backes, H., Neubauer, F., Dougherty, M., Achilleos, N., Andre, N., Arridge, C., Bertucci, C., Jones, G., Khurana, K., Russell, C., and Wennmacher, A. (2005) Titan's magnetic field signature during the first Cassini encounter. Science 308:992-995.

Bagshaw, M. (2008) Cosmic radiation in commercial aviation. Travel Med Infect Dis 6:125-127.

Bailer-Jones, C. (2009) The evidence for and against astronomical impacts on climate change and mass extinctions: a review. International Journal of Astrobiology 8:213-219.

Baltschukat, K. and Horneck, G. (1991) Responses to accelerated heavy-ions of spores of Bacillus subtilis of different repair capacity. Radiat Environ Biophys 30:87-103.

Banaszkiewicz, M., Lara, L., Rodrigo, R., López-Moreno, J.J., and Molina-Cuberos, G.J. (2000) A coupled model of Titan's atmosphere and ionosphere. Icarus 147:386-404.

Battista, J. (1997) Against all odds: the survival strategies of Deinococcus radiodurans. Annu Rev Microbiol 51:203-224.

Battista, J., Earl, A., and Park, M.-J. (1999) Why is Deinococcus radiodurans so resistant to ionizing radiation? Trends Microbiol 7:362-365.

Bauleo, P.M. and Martino, J.R. (2009) The dawn of the particle astronomy era in ultra-high-energy cosmic rays. Nature 458:847-851.

Baumstark-Khan, C. and Facius, R. (2001) Life under conditions of ionizing radiation. In Astrobiology: The Quest for the Conditions of Life, edited by G. Horneck and C. Baumstark-Khan, Springer, Berlin, pp 260-283.

Bazilevskaya, G., Krainev, M., and Makhmutov, V. (2000) Effects of cosmic rays on the Earth's environment. Journal of Atmospheric and Solar-Terrestrial Physics 62:1577-1586.

Bazilevskaya, G., Usoskin, I., Flückiger, E., Harrison, R., Desorgher, L., Bütikofer, R., Krainev, M., Makhmutov, V., Stozhkov, Y., Svirzhevskaya, A., Svirzhevsky, N., and Kovaltsov, G. (2008) Cosmic ray induced ion production in the atmosphere. Space Sci Rev 137:149-173.

Beatty, J., Overmann, J., Lince, M., Manske, A., Lang, A., Blankenship, R., Van Dover, C., Martinson, T., and Plumley, F. (2005) An obligately photosynthetic bacterial anaerobe from a deep-sea hydrothermal vent. Proc Natl Acad Sci USA 102:93069310.

Beltrán, M.T., Codella, C., Viti, S., Neri, R., and Cesaroni, R. (2009) First detection of glycolaldehyde outside the Galactic Center. Astrophys J 690:L93-L96.
Benner, S.A., Kim, H.-J., Kim, M.-J., and Ricardo, A. (2010) Planetary organic chemistry and the origins of biomolecules. Cold Spring Harb Perspect Biol 2, doi:10.1101/cshperspect. a003467.

Benton, E.R. and Benton, E.V. (2001) Space radiation dosimetry in low-Earth orbit and beyond. Nucl Instrum Methods Phys Res B 184:255-294.

Berger, W.H. (1990) The Younger Dryas cold spell—a quest for causes. Glob Planet Change 3:219-237.

Bird, M., Dutta-Roy, R., Asmar, S., and Rebold, T. (1997) Detection of Titan's ionosphere from Voyager 1 radio occultation observations. Icarus 130:426-436.

Blandford, R. and Eichler, D. (1987) Particle acceleration at astrophysical shocks: a theory of cosmic ray origin. Phys Rep 154:1-75.

Bolaños, L., Lukaszewski, K., Bonilla, I., and Blevins, D. (2004) Why boron? Plant Physiol Biochem 42:907-912.

Bonner, W.A. (2000) Parity violation and the evolution of biomolecular homochirality. Chirality 12:114-126.

Borucki, W.J., Levin, Z., Whitten, R.C., Keesee, R.G., Capone, L.A., Toon, O.B., and Dubach, J. (1982) Predicted electrical conductivity between 0 and $80 \mathrm{~km}$ in the venusian atmosphere. Icarus 51:302-321.

Borucki, W.J., Levin, Z., Whitten, R.C., Keesee, R.G., Capone, L.A., Summers, A.L., Toon, O.B., and Dubach, J. (1987) Predictions of the electrical conductivity and charging of the aerosols in Titan's atmosphere. Icarus 72:604-622.

Borucki, W.J., Whitten, R.C., Bakes, E.L.O., Barth, E., and Tripathi, S. (2006) Predictions of the electrical conductivity and charging of the aerosols in Titan's atmosphere. Icarus 181:527-544.

Boyd, R.N., Kajino, T., and Onaka, T. (2010) Supernovae and the chirality of the amino acids. Astrobiology 10:561-568.

Brack, A., Horneck, G., Cockell, C.S., Bérces, A., Belisheva, N.K., Eiroa, C., Henning, T., Herbst, T., Kaltenegger, L., Léger, A., Liseau, R., Lammer, H., Selsis, F., Beichman, C., Danchi, W., Fridlund, M., Lunine, J., Paresce, F., Penny, A., Quirrenbach, A., Röttgering, H., Schneider, J., Stam, D., Tinetti, G., and White, G. (2010) Origin and evolution of life on terrestrial planets. Astrobiology 10:69-76.

Brakenridge, G.R. (1981) Terrestrial paleoenvironmental effects of a late quaternary-age supernova. Icarus 46:81-93.

Bromm, V., Coppi, P., and and Larson, R. (2002) The formation of the first stars. I. The primordial star-forming cloud. Astrophys J 564:23-51.

Bromm, V., Yoshida, N., Hernquist, L., and McKee, C. (2009) The formation of the first stars and galaxies. Nature 459:49-54.

Brown, R.H., Lebreton, J.-P., and Waite, J.H. (2009) Titan from Cassini-Huygens, Springer, New York.

Burchell, M.J. (2004) Panspermia today. International Journal of Astrobiology 3:73-80.

Butt, Y. (2009) Beyond the myth of the supernova-remnant origin of cosmic rays. Nature 460:701-704.

Cane, H. (2000) Coronal mass ejections and Forbush decreases. Space Sci Rev 93:55-77.

Capone, L., Dubach, J., Whitten, R., Prasad, S., and Santhanam, K. (1980) Cosmic ray synthesis of organic molecules in Titan's atmosphere. Icarus 44:72-84.

Carslaw, K., Harrison, R., and Kirkby, J. (2002) Cosmic rays, clouds, and climate. Science 298:1732-1737.

Charnley, S., Rodgers, S., Kuan, Y., and Huang, H. (2002) Biomolecules in the interstellar medium and in comets. Adv Space Res 30:1419-1431.

Chyba, C. (2000a) Energy for microbial life on Europa. Nature 403:381-382. 
Chyba, C. (2000b) Correction: Energy for microbial life on Europa. Nature 406:368.

Chyba, C. and Hand, K. (2001) Life without photosynthesis. Science 292:2026-2027.

Chyba, C. and Phillips, C. (2001) Possible ecosystems and the search for life on Europa. Proc Natl Acad Sci USA 98:801-804.

Clark, B., Baker, A., Cheng, A., Clemett, S., McKay, D., McSween, H., Pieters, C., Thomas, P., and Zolensky, M. (1999) Survival of life on asteroids, comets and other small bodies. Orig Life Evol Biosph 29:521-545.

Clark, B.C. (2001) Planetary interchange of bioactive material: probability factors and implications. Orig Life Evol Biosph 31:185-197.

Clarke, J. (1981) Extraterrestrial intelligence and galactic nuclear activity. Icarus 46:94-96.

Clowdsley, M.S., Wilson, J.W., Kim, M.-H.Y., Singleterry, R.C., Tripathi, R.K., Heinbockel, J.H., Badavi, F.F., and Shinn, J.L. (2001) Neutron environments on the martian surface. Phys Med 17:94-96.

Coates, A.J., Crary, F.J., Lewis, G.R., Young, D.T., Waite, J.H., and Sittler, E.C. (2007) Discovery of heavy negative ions in Titan's ionosphere. Geophys Res Lett 34:L22103.

Cockell, C. (1999) Life on Venus. Planet Space Sci 47:1487-1501.

Cockell, C.S. (2008) Interstellar planetary protection. Adv Space Res 42:1161-1165.

Collins, C., Albarrán, G., and Collins, K. (2000) Irradiation promoted production of organic precursor species in inorganic solids on the prebiotic Earth. In The Role of Radiation in the Origin and Evolution of Life, edited by M. Akaboshi, N. Fujii, and R. Navarro-González, Kyoto University Press, Kyoto, pp 143-153.

Committee on the Evaluation of Radiation Shielding for Space Exploration. (2008) Managing Space Radiation Risk in the New Era of Space Exploration, National Academies Press, Washington DC. Available online at http://www.nap.edu/catalog .php?record_id=12045.

Committee on the Limits of Organic Life in Planetary Systems. (2007) The Limits of Organic Life in Planetary Systems, National Academies Press, Washington DC. Available online at http:// www.nap.edu/catalog/11919.html.

Committee on the Origins and Evolution of Life. (2005) The Astrophysical Context of Life, National Academies Press, Washington DC. Available online at http://www.nap.edu/ catalog/11316.html.

Cooper, J.F., Johnson, R.E., Mauk, B.H., Garrett, H.B., and Gehrels, N. (2001) Energetic ion and electron irradiation of the icy Galilean satellites. Icarus 149:133-159.

Court, R., Sephton, M., Parnell, J., and Gilmour, I. (2006) The alteration of organic matter in response to ionising irradiation: chemical trends and implications for extraterrestrial sample analysis. Geochim Cosmochim Acta 70:1020-1039.

Cox, M. and Battista, J. (2005) Deinococcus radiodurans-the consummate survivor. Nat Rev Microbiol 3:882-892.

Cravens, T., Vann, J., Clark, J., Yu, J., Keller, C., and Brull, C. (2004) The ionosphere of Titan: an updated theoretical model. Adv Space Res 33:212-215.

Cravens, T.E., Robertson, I.P., Waite, J.H., Yelle, R.V., Kasprzak, W.T., Keller, C.N., Ledvina, S.A., Niemann, H.B., Luhmann, J.G., McNutt, R.L., Ip, W.-H., De La Haye, V., MuellerWodarg, I., Wahlund, J.-E., Anicich, V.G., and Vuitton, V. (2006) Composition of Titan's ionosphere. Geophys Res Lett 33:L07105.

Crawford, I., Fagents, S., Joy, K., and Rumpf, M. (2010) Lunar palaeoregolith deposits as recorders of the galactic environ- ment of the Solar System and implications for astrobiology. Earth Moon Planets 107:75-85.

Crutzen, P.J. and Brühl, C. (1996) Mass extinctions and supernova explosions. Proc Natl Acad Sci USA 93:1582-1584.

Cucinotta, F., Saganti, P., Wilson, J., and Simonsen, L. (2002) Model predictions and visualization of the particle flux on the surface of Mars. J Radiat Res (Tokyo) 43:S35-S39.

Dadachova, E., Bryan, R., Huang, X., Moadel, T., Schweitzer, A., Aisen, P., Nosanchuk, J., and Casadevall, A. (2007) Ionizing radiation changes the electronic properties of melanin and enhances the growth of melanized fungi. PLOS ONE 2:e457.

Dalgarno, A. (2006) The galactic cosmic ray ionization rate. Proc Natl Acad Sci USA 103:12269-12273.

Daly, M., Gaidamakova, E., Matrosova, V., Vasilenko, A., Zhai, M., Venkateswaran, A., Hess, M., Omelchenko, M., Kostandarithes, H., Makarova, K., Wackett, L., Fredrickson, J., and Ghosal, D. (2004) Accumulation of Mn(II) in Deinococcus radiodurans facilitates gamma-radiation resistance. Science 306: $1025-1028$.

Daly, M.J. (2009) A new perspective on radiation resistance based on Deinococcus radiodurans. Nat Rev Microbiol 7:237-245.

Dar, A., Laor, A., and Shaviv, N. (1998) Life extinctions by cosmic ray jets. Phys Rev Lett 80:5813-5816.

Dartnell, L., Desorgher, L., Ward, J., and Coates, A. (2007a) Modelling the surface and subsurface martian radiation environment: implications for astrobiology. Geophys Res Lett 34:L02207.

Dartnell, L.R., Desorgher, L., Ward, J.M., and Coates, A.J. (2007b) Martian sub-surface ionising radiation: biosignatures and geology. Biogeosciences 4:545-558.

Dartnell, L.R., Hunter, S., Lovell, K., Coates, A., and Ward, J. (2010) Low-temperature ionizing radiation resistance of Deinococcus radiodurans and Antarctic Dry Valley bacteria. Astrobiology 10:717-732.

De Angelis, G., Clowdsley, M.S., Singleterry, R.C., and Wilson, J.W. (2004) A new Mars radiation environment model with visualization. Adv Space Res 34:1328-1332.

de La Vega, U., Rettberg, P., and Reitz, G. (2007) Simulation of the environmental climate conditions on martian surface and its effect on Deinococcus radiodurans. Adv Space Res 40:1672-1677.

Delitsky, M. and Lane, A. (1998) Ice chemistry on the Galilean satellites. J Geophys Res 103:31391-31403.

Dermer, C.D. and Holmes, J.M. (2005) Cosmic rays from gamma ray bursts in the Galaxy. Astrophys J 628:L21-L24.

Des Marais, D., Harwit, M., Jucks, K., Kasting, J., Lin, D., Lunine, J., Schneider, J., Seager, S., Traub, W., and Woolf, N. (2002) Remote sensing of planetary properties and biosignatures on extrasolar terrestrial planets. Astrobiology 2:153-181.

Desch, S. and Mouschovias, T. (2001) The magnetic decoupling stage of star formation. Astrophys J 550:314-333.

Draganic, I. (2005) Radiolysis of water: a look at its origin and occurrence in the nature. Radiation Physics and Chemistry 72:181-186.

Draganic, I., Draganic, Z., and Altiparmakov, D. (1983) Natural nuclear reactors and ionizing radiation in the Precambrian. Precambrian Res 20:283-298.

Draganic, I.G. (2000) Radiation chemical approaches to chemical evolution processes on Earth and beyond. In The Role of $R a-$ diation in the Origin and Evolution of Life, edited by M. Akaboshi, N. Fujii, and R. Navarro-González, Kyoto University Press, Kyoto, pp 53-72.

Dubach, J., Whitten, R.C., and Sims, J.S. (1974) The lower ionosphere of Venus. Planet Space Sci 22:525-536. 
Dwyer, J. (2005) The initiation of lightning by runaway air breakdown. Geophys Res Lett 32:L20808.

Ehrenfreund, P. and Charnley, S. (2000) Organic molecules in the interstellar medium, comets, and meteorites: a voyage from dark clouds to the early Earth. Annu Rev Astron Astrophys 38: 427-483.

Ejzak, L., Melott, A., Medvedev, M., and Thomas, B. (2007) Terrestrial consequences of spectral and temporal variability in ionizing photon events. Astrophys J 654:373-384.

Ellis, J. and Schramm, D. (1995) Could a nearby supernova explosion have caused a mass extinction? Proc Natl Acad Sci USA 92:235-238.

Elser, J., Bracken, M., Cleland, E., Gruner, D., Harpole, W., Hillebrand, H., Ngai, J., Seabloom, E., Shurin, J., and Smith, J. (2007) Global analysis of nitrogen and phosphorus limitation of primary producers in freshwater, marine and terrestrial ecosystems. Ecol Lett 10:1135-1142.

Erlykin, A. and Wolfendale, A. (2001) Supernova remnants and the origin of the cosmic radiation: II. Spectral variations in space and time. J Phys G Nucl Part Phys 27:959-976.

Eugster, O. (2003) Cosmic-ray exposure ages of meteorites and lunar rocks and their significance. Chemie der Erde - Geochemistry 63:3-30.

Ewing, D. (1995) The directed evolution of radiation resistance in E. coli. Biochem Biophys Res Commun 216:549-553.

Ewing, D. (1997) Production of radiation-resistant E. coli strains by daily X-irradiation. Int J Radiat Biol 71:253-258.

Fagents, S.A., Rumpf, M.E., Crawford, I.A., and Joy, K.H. (2010) Preservation potential of implanted solar wind volatiles in lunar paleoregolith deposits buried by lava flows. Icarus 207:595-604.

Fahr, H. (2004) Global structure of the heliosphere and interaction with the local interstellar medium: three decades of growing knowledge. Adv Space Res 34:3-13.

Fajardo-Cavazos, P., Langenhorst, F., Melosh, H., and Nicholson, W. (2009) Bacterial spores in granite survive hypervelocity launch by spallation: implications for lithopanspermia. Astrobiology 9:647-657.

Federman, S.R., Weber, J., and Lambert, D. (1996) Cosmic rayinduced chemistry towards Perseus OB2. Astrophys J 436: 181-190.

Feldman, W., Boynton, W., Tokar, R., Prettyman, T., Gasnault, O., Squyres, S., Elphic, R., Lawrence, D., Lawson, S., Maurice, S., McKinney, G., Moore, K., and Reedy, R. (2002) Global distribution of neutrons from Mars: results from Mars Odyssey. Science 297:75-78.

Ferrari, F. and Szuszkiewicz, E. (2009) Cosmic rays-a review for astrobiologists. Astrobiology 9:413-436.

Fields, B.D., Athanassiadou, T., and Johnson, S.R. (2008) Supernova collisions with the heliosphere. Astrophys J 678:549-562.

Fitz, D., Reiner, H., Plankensteiner, K., and Rode, B.M. (2007) Possible origins of biohomochirality. Curr Chem Biol 1:41-52.

Fraústo da Silva, J. and Williams, R. (2001) The Biological Chemistry of the Elements: The Inorganic Chemistry of Life, $2^{\text {nd }}$ ed., Oxford University Press, Oxford.

Gal-Yam, A., Mazzali, P., Ofek, E., Nugent, P., Kulkarni, S., Kasliwal, M., Quimby, R., Filippenko, A., Cenko, S., Chornock, R., Waldman, R., Kasen, D., Sullivan, M., Beshore, E., Drake, A., Thomas, R., Bloom, J., Poznanski, D., Miller, A., Foley, R., Silverman, J., Arcavi, I., Ellis, R., and Deng, J. (2009) Supernova 2007bi as a pair-instability explosion. Nature 462:624-627.

Galante, D. and Horvath, J.E. (2007a) Biological effects of gamma-ray bursts: distances for severe damage on the biota. International Journal of Astrobiology 6:19-26.
Galante, D. and Horvath, J.E. (2007b) Quantitative biological effects of gamma-ray bursts. International Journal of Modern Physics D 16:509-514.

Garzón, L. and Garzón, M. (2001) Radioactivity as a significant energy source in prebiotic synthesis. Orig Life Evol Biosph 31:3-13.

Gauthier-Lafaye, F. and Weber, F. (2003) Natural nuclear fission reactors: time constraints for occurrence, and their relation to uranium and manganese deposits and to the evolution of the atmosphere. Precambrian Res 120:81-100.

Gauthier-Lafaye, F., Holliger, P., and Blanc, P. (1996) Natural fission reactors in the Franceville basin, Gabon: a review of the conditions and results of a "critical event" in a geologic system. Geochim Cosmochim Acta 60:4831-4852.

Gehrels, N., Laird, C., Jackman, C., Cannizzo, J., Mattson, B., and Chen, W. (2003) Ozone depletion from nearby supernovae. Astrophys J 585:1169-1176.

Ghosal, D., Omelchenko, M., Gaidamakova, E., Matrosova, V., Vasilenko, A., Venkateswaran, A., Zhai, M., Kostandarithes, H., Brim, H., Makarova, K., Wackett, L., Fredrickson, J., and Daly, M. (2005) How radiation kills cells: survival of Deinococcus radiodurans and Shewanella oneidensis under oxidative stress. FEMS Microbiol Rev 29:361-375.

Giacalone, J. and Kóta, J. (2006) Acceleration of solar-energetic particles by shocks. Space Sci Rev 124:277-288.

Giraud, A., Matic, I., Tenaillon, O., Clara, A., Radman, M., Fons, M., and Taddei, F. (2001) Costs and benefits of high mutation rates: adaptive evolution of bacteria in the mouse gut. Science 291:2606-2608.

Gladman, B., Dones, L., Levison, H., and Burns, J. (2005) Impact seeding and reseeding in the inner Solar System. Astrobiology 5:483-496.

Glassmeier, K.-H. and Vogt, J. (2010) Magnetic polarity transitions and biospheric effects. Space Sci Rev 155:387-410.

Greenberg, R. (2010) Transport rates of radiolytic substances into Europa's ocean: implications for the potential origin and maintenance of life. Astrobiology 10:275-283.

Greenberg, R., Geissler, P., Tufts, B., and Hoppa, G. (2000) Habitability of Europa's crust: the role of tidal-tectonic processes. J Geophys Res 105:17551-17562.

Grenfell, J., Grießmeier, J.-M., Patzer, B., Rauer, H., Segura, A., Stadelmann, A., Stracke, B., Titz, R., and Von Paris, P. (2007) Biomarker response to galactic cosmic ray-induced $\mathrm{NO}_{\mathrm{x}}$ and the methane greenhouse effect in the atmosphere of an Earthlike planet orbiting an M dwarf star. Astrobiology 7:208-221.

Grew, E., Bada, J., and Hazen, R. (2011) Borate minerals and origin of the RNA World. Orig Life Evol Biosph doi:10.1007/ s11084-010-9233-y.

Grießmeier, J.-M., Stadelmann, A., Motschmann, U., Belisheva, N., Lammer, H., and Biernat, H. (2005) Cosmic ray impact on extrasolar Earth-like planets in close-in habitable zones. Astrobiology 5:587-603.

Grießmeier, J.-M., Stadelmann, A., Grenfell, J.L., Lammer, H., and Motschmann, U. (2009) On the protection of extrasolar Earth-like planets around $\mathrm{K} / \mathrm{M}$ stars against galactic cosmic rays. Icarus 199:526-535.

Gurevich, A., Zybin, K., and Roussel-Dupre, R. (1999) Lightning initiation by simultaneous effect of runaway breakdown and cosmic ray showers. Phys Lett A 254:79-87.

Head, J., Pappalardo, R., and Sullivan, R. (1999) Europa: morphological characteristics of ridges and triple bands from Galileo data (E4 and E6) and assessment of a linear diapirism model. J Geophys Res 104:24223-24236.

Herbst, E. and van Dishoeck, E. (2009) Complex organic interstellar molecules. Annu Rev Astron Astrophys 47:427-480. 
Hollis, J.M., Lovas, F.J., and Jewell, P.R. (2000) Interstellar glycolaldehyde: the first sugar. Astrophys J 540:L107-L110.

Horneck, G. (1995) Quantification of the biological effectiveness of environmental UV radiation. J Photochem Photobiol B 31:43-49.

Horneck, G. (2000) The microbial world and the case for Mars. Planet Space Sci 48:1053-1063.

Horneck, G., Bücker, H., and Reitz, G. (1994) Long-term survival of bacterial spores in space. Adv Space Res 14:41-45.

Horneck, G., Rettberg, P., Reitz, G., Wehner, J., Eschweiler, U., Strauch, K., Panitz, C., Starke, V., and Baumstark-Khan, C. (2001) Protection of bacterial spores in space, a contribution to the discussion on panspermia. Orig Life Evol Biosph 31:527-547.

Horneck, G., Klaus, D., and Mancinelli, R. (2010) Space microbiology. Microbiol Mol Biol Rev 74:121-156.

ICRP. (1991) 1990 Recommendations of the International Commission on Radiological Protection. Annals of the ICRP 21(1-3): $1-201$.

Imlay, J.A. (2003) Pathways of oxidative damage. Annu Rev Microbiol 57:395-418.

Indriolo, N., Geballe, T., Oka, T., and McCall, B. (2007) $\mathrm{H}_{3}^{+}$in diffuse interstellar clouds: a tracer for the cosmic-ray ionization rate. Astrophys J 671:1736-1747.

Ip, W.-H. (1990) Titan's upper ionosphere. Astrophys J 362: 354-363.

Irwin, L. and Schulze-Makuch, D. (2003) Strategy for modeling putative multilevel ecosystems on Europa. Astrobiology 3: 813-821.

Israel, M., George, J., Mewaldt, R., and deNolfo, G. (2004) An overview of cosmic-ray elemental composition. Advanced Composition Explorer (ACE) News \#83. Available online at http://www.srl.caltech.edu/ACE/ACENews/ACENews83 .html.

Itoh, T., Martin, W., and Nei, M. (2002) Acceleration of genomic evolution caused by enhanced mutation rate in endocellular symbionts. Proc Natl Acad Sci USA 99:12944-12948.

Jackman, C.H., Fleming, E.L., and Vitt, F.M. (2000) Influence of extremely large solar proton events in a changing stratosphere. J Geophys Res 105:11659-1670.

Jensen, K.A. and Ewing, R.C. (2001) The Okélobondo natural fission reactor, southeast Gabon: geology, mineralogy, and retardation of nuclear-reaction products. Geol Soc Am Bull 113:32-62.

Jolivet, E., L'Haridon, S., Corre, E., Forterre, P., and Prieur, D. (2003) Thermococcus gammatolerans sp. nov., a hyperthermophilic archaeon from a deep-sea hydrothermal vent that resists ionizing radiation. Int J Syst Evol Microbiol 53:847-851.

Jolivet, E., Corre, E., L'Haridon, S., Forterre, P., and Prieur, D. (2004) Thermococcus marinus sp. nov. and Thermococcus radiotolerans sp. nov., two hyperthermophilic archaea from deepsea hydrothermal vents that resist ionizing radiation. Extremophiles 8:219-227.

Kaltenegger, L., Traub, W., and and Jucks, K. (2007) Spectral evolution of an Earth-like planet. Astrophys J 658:598-616.

Karam, P.A. and Leslie, S. (1999) Calculations of background beta-gamma radiation dose through geologic time. Health Phys 77:662-667.

Karam, P.A., Leslie, S.A., and Anbar, A. (2001) The effects of changing atmospheric oxygen concentrations and background radiation levels on radiogenic DNA damage rates. Health Phys 81:545-553.

Keller, C.N., Anicich, V.G., and Cravens, T.E. (1998) Model of Titan's ionosphere with detailed hydrocarbon ion chemistry. Planet Space Sci 46:1157-1174.

Khodachenko, M., Ribas, I., Lammer, H., Grießmeier, J.-M., Leitner, M., Selsis, F., Eiroa, C., Hanslmeier, A., Biernat, H.,
Farrugia, C., and Rucker, H. (2007) Coronal mass ejection (CME) activity of low mass $\mathrm{M}$ stars as an important factor for the habitability of terrestrial exoplanets. I. CME impact on expected magnetospheres of Earth-like exoplanets in close-in habitable zones. Astrobiology 7:167-184.

Kiefer, J. (1990) Biological Radiation Effects, Springer-Verlag, Heidelberg.

Kim, M.-H., Thibeault, S., Simonsen, L., and Wilson, J. (1998) Comparison of martian meteorites and martian regolith as shield materials for galactic cosmic rays. NASA/TP-1998208724, National Aeronautics and Space Administration, Washington DC. Available online at http://citeseerx.ist. psu.edu/viewdoc/download?doi $=10.1 \cdot 1.35 .9576 \&$ rep $=$ rep1\& type $=$ pdf.

Kirkby, J. (2007) Cosmic rays and climate. Surveys in Geophysics 28:333-375.

Kivelson, M., Khurana, K., Russell, C., Walker, R., Warnecke, J., Coroniti, F., Polanskey, C., Southwood, D., and Schubert, G. (1996) Discovery of Ganymede's magnetic field by the Galileo spacecraft. Nature 384:537-541.

Kivelson, M.G. and Russell, C.T., editors. (1995) Introduction to Space Physics, Cambridge University Press, Cambridge.

Klapdor-Kleingrothaus, H.V. and Zuber, K. (2000) Chapter 8: cosmic radiation. In Particle Astrophysics, Institute of Physics Publishing, Bristol, pp 223-247.

Kminek, G. and Bada, J. (2006) The effect of ionizing radiation on the preservation of amino acids on Mars. Earth Planet Science Lett 245:1-5.

Kminek, G., Bada, J., Pogliano, K., and Ward, J. (2003) Radiationdependent limit for the viability of bacterial spores in halite fluid inclusions and on Mars. Radiat Res 159:722-729.

Knie, K., Korschinek, G., Faestermann, T., Wallner, C., Scholten, J., and Hillebrandt, W. (1999) Indication for supernova produced Fe-60 activity on Earth. Phys Rev Lett 83:18-21.

Knie, K., Korschinek, G., Faestermann, T., Dorfi, E., Rugel, G., and Wallner, A. (2004) Fe-60 anomaly in a deep-sea manganese crust and implications for a nearby supernova source. Phys Rev Lett 93, doi:10.1103/PhysRevLett.93.171103.

Kobayashi, K. and Tsuji, T. (1997) Abiotic synthesis of uracil from carbon monoxide, nitrogen and water by proton irradiation. Chemistry Letters 26:903-904.

Kobayashi, K., Kaneko, T., Saito, T., and Oshima, T. (1998) Amino acid formation in gas mixtures by high energy particle irradiation. Orig Life Evol Biosph 28:155-165.

Kobayashi, K., Kaneko, T., and Saito, T. (1999) Characterization of complex organic compounds formed in simulated planetary atmospheres by the action of high energy particles. Adv Space Res 24:461-464.

Kolomnikov, I., Lysyak, T.V., Konash, E., Kalyazin, E.P., Rudnev, A.V., and Kharitonov, Y.Y. (1982) Formation of organic products from metal carbonates and water by the action of ionizing radiation. Doklady Physical Chemistry (English Translation) 265:912-913.

Krasnopolsky, V. (2009) A photochemical model of Titan's atmosphere and ionosphere. Icarus 201:226-256.

Kuan, Y.-J., Charnley, S., Huang, H.-C., Tseng, W.-L., and and Kisiel, Z. (2003) Interstellar glycine. Astrophys J 593: 848-867.

Kuznetsov, N.V., Nymmik, R.A., and Panasyuk, M.I. (2001) The balance between fluxes of galactic cosmic rays and solar energetic particles, depending on solar activity. In Proceedings of the $27^{\text {th }}$ International Cosmic Ray Conference. Hamburg, Germany, International Union of Pure and Applied Physics, London, Vol. 8, pp 3193-3196. 
Lammer, H., Bredehöft, J., Coustenis, A., Khodachenko, M., Kaltenegger, L., Grasset, O., Prieur, D., Raulin, F., Ehrenfreund, P., Yamauchi, M., Wahlund, J., Grießmeier, J., Stangl, G., Cockell, C., Kulikov, Y., Grenfell, J., and Rauer, H. (2009) What makes a planet habitable? Astronomy and Astrophysics Review 17:181-249.

Larson, R.B. (2003) The physics of star formation. Rep Prog Phys 66:1651-1697.

Larson, R.B. (2010) Angular momentum and the formation of stars and black holes. Rep Prog Phys 73, doi:10.1088/00344885/73/1/014901.

Laut, P. (2003) Solar activity and terrestrial climate: an analysis of some purported correlations. Journal of Atmospheric and Solar-Terrestrial Physics 65:801-812.

Le Postollec, A., Incerti, S., Dobrijevic, M., Desorgher, L., Santin, G., Moretto, P., Vandenabeele-Trambouze, O., Coussot, G., Dartnell, L., and Nieminen, P. (2009a) Monte-Carlo simulation of the radiation environment encountered by a biochip during a space mission to Mars. Astrobiology 9:311-323.

Le Postollec, A., Coussot, G., Baqué, M., Incerti, S., Desvignes, I., Moretto, P., Dobrijevic, M., and Vandenabeele-Trambouze, O. (2009b) Investigation of neutron radiation effects on polyclonal antibodies (IgG) and fluorescein dye for astrobiological applications. Astrobiology 9:637-645.

Lieberman, B.S. and Melott, A.L. (2007) Considering the case for biodiversity cycles: re-examining the evidence for periodicity in the fossil record. PLOS ONE 2:e759.

Lim, M. (2002) Cosmic rays: are air crew at risk? Occup Environ Med 59:428-432.

Lin, L.-H., Wang, P.-L., Rumble, D., Lippmann-Pipke, J., Boice, E., Pratt, L., Lollar, B., Brodie, E., Hazen, T., Andersen, G., DeSantis, T., Moser, D., Kershaw, D., and Onstott, T. (2006) Long-term sustainability of a high-energy, low-diversity crustal biome. Science 314:479-482.

Lineweaver, C., Fenner, Y., and Gibson, B. (2004) The galactic habitable zone and the age distribution of complex life in the Milky Way. Science 303:59-62.

Lovelock, J.E. (1965) A physical basis for life detection experiments. Nature 207:568-570.

Lucey, P. (2000) Potential for prebiotic chemistry at the poles of the Moon. Proc SPIE 4137:84-88.

Luckey, T.D. (2008) The evidence for gamma ray photosynthesis. 21st Century Science \& Technology, Fall-Winter 2008, pp 4-6. Available online at http://www.21stcenturysciencetech.com/ Articles\%202008/F-W_2008/Research_Communication.pdf.

Lyons, L.R. (1997) Magnetospheric processes leading to precipitation. Space Sci Rev 80:109-132.

Makarova, K., Aravind, L., Wolf, Y., Tatusov, R., Minton, K., Koonin, E., and Daly, M. (2001) Genome of the extremely radiation-resistant bacterium Deinococcus radiodurans viewed from the perspective of comparative genomics. Microbiol Mol Biol Rev 65:44-79.

Martín, O., Galante, D., Cárdenas, R., and Horvath, J. (2009) Short-term effects of gamma ray bursts on Earth. Astrophys Space Sci 321:161-167.

Martin-Torres, F. and Molina-Cuberos, G. (2002) Ion-neutral model of the lower ionosphere of Venus [abstract P21A-11]. In AGU Spring Meeting Abstracts, American Geophysical Union, Washington DC.

McKee, C. and Ostriker, E. (2007) Theory of star formation. Annu Rev Astron Astrophys 45:565-687.

Medvedev, M. and Melott, A. (2007) Do extragalactic cosmic rays induce cycles in fossil diversity? Astrophysical J 664: 879-889.
Melosh, H. (1984) Impact ejection, spallation, and the origin of meteorites. Icarus 59:234-260.

Melosh, H. (2004) Exchange of meteorites (and life?) between stellar systems. Astrobiology 3:207-215.

Melott, A.L. and Thomas, B.C. (2009) Late Ordovician geographic patterns of extinction compared with simulations of astrophysical ionizing radiation damage. Paleobiology 35: 311-320.

Melott, A., Lieberman, B., Laird, C., Martin, L., Medvedev, M., Thomas, B., Cannizzo, J., Gehrels, N., and Jackman, C. (2004) Did a gamma-ray burst initiate the late Ordovician mass extinction? International Journal of Astrobiology 3:55-61.

Melott, A., Thomas, B., Hogan, D., Ejzak, L., and Jackman, C. (2005) Climatic and biogeochemical effects of a galactic gamma ray burst. Geophys Res Lett 32:L14808.

Melott, A., Krejci, A., Thomas, B., Medvedev, M., Wilson, G., and Murray, M. (2008) Atmospheric consequences of cosmicray variability in the extragalactic shock model. J Geophys Res 113, doi:10.1029/2008JE003206.

Meneguzzi, M., Audouze, J., and Reeves, H. (1971) The production of the elements $\mathrm{Li}, \mathrm{Be}, \mathrm{B}$ by galactic cosmic rays in space and its relation with stellar observations. Astron Astrophys 15:337-359.

Mewaldt, R. (2006) Solar energetic particle composition, energy spectra, and space weather. Space Sci Rev 124:303-316.

Mileikowsky, C., Cucinotta, F., Wilson, J., Gladman, B., Horneck, G., Lindegren, L., Melosh, J., Rickman, H., Valtonen, M., and Zheng, J. (2000) Natural transfer of viable microbes in space. 1. From Mars to Earth and Earth to Mars. Icarus 145:391-427.

Minton, K. (1994) DNA repair in the extremely radioresistant bacterium Deinococcus radiodurans. Mol Microbiol 13:9-15.

Mitrofanov, I., Anfimov, D., Kozyrev, A., Litvak, M., Sanin, A., Tret'yakov, V., Krylov, A., Shvetsov, V., Boynton, W., Shinohara, C., Hamara, D., and Saunders, R. (2002) Maps of subsurface hydrogen from the High Energy Neutron Detector, Mars Odyssey. Science 297:78-81.

Moeller, R., Rohde, M., and Reitz, G. (2010) Effects of ionizing radiation on the survival of bacterial spores in artificial martian regolith. Icarus 206:783-786.

Molina-Cuberos, G., Lopez-Moreno, J., Rodrigo, R., and Lara, L. (1999a) Chemistry of the galactic cosmic ray induced ionosphere of Titan. J Geophys Res 104:21997-22024.

Molina-Cuberos, G., Lopez-Moreno, J., Rodrigo, R., Lara, L., and O'Brien, K. (1999b) Ionization by cosmic rays of the atmosphere of Titan. Planet Space Sci 47:1347-1354.

Molina-Cuberos, G.J., Stumptner, W., Lammer, H., Komle, N.I., and O'Brien, K. (2001) Cosmic ray and UV radiation models on the ancient martian surface. Icarus 154:216-222.

Moore, M., Hudson, R., and Gerakines, P. (2001) Mid- and farinfrared spectroscopic studies of the influence of temperature, ultraviolet photolysis and ion irradiation on cosmic-type ices. Spectrochim Acta A Mol Biomol Spectrosc 57:843-858.

Nagy, B., Gauthier-Lafaye, F., Holliger, P., Davis, D., Mossman, D., Leventhal, J., Rigali, M., and Parnell, J. (1991) Organic matter and containment of uranium and fissiogenic isotopes at the Oklo natural reactors. Nature 354:472-475.

NCRP. (1993) Limitation of Exposure to Ionizing Radiation, NCRP Report No. 116, National Council on Radiation Protection and Measurements, Bethesda, MD. Available online at http:// www.ncrponline.org/Publications/116press.html.

NCRP. (2006) Information Needed to Make Radiation Protection Recommendations for Space Missions Beyond Low-Earth Orbit, NCRP Report No. 153, National Council on Radiation Protection and 
Measurements, Bethesda, MD. Available online at http:// www.ncrponline.org/Publications/153press.html.

Nelson, G.A. (2003) Fundamental space radiobiology. Gravit Space Biol Bull 16:29-36.

Nicholson, W. (2009) Ancient micronauts: interplanetary transport of microbes by cosmic impacts. Trends Microbiol 17:243-250.

Nielsen, F.H. (1998) Ultratrace elements in nutrition: current knowledge and speculation. The Journal of Trace Elements in Experimental Medicine 11:251-274.

Nyquist, L. (1983) Do oblique impacts produce martian meteorites? J Geophys Res 88(Supplement):A785-A798.

O'Brien, K., Friedberg, W., Sauer, H., and Smart, D. (1996) Atmospheric cosmic rays and solar energetic particles at aircraft altitudes. Environ Int 22:9-44.

Öberg, K.I., Fuchs, G.W., Awad, Z., Fraser, H.J., Schlemmer, S., van Dishoeck, E.F., and Linnartz, H. (2007) Photodesorption of CO Ice. Astrophys J 662:L23-L26.

Overholt, A.C., Melott, A.L., and Pohl, M. (2009) Testing the link between terrestrial climate change and galactic spiral arm transit. The Astrophysical Journal Letters 705:L101-L103.

Padovani, M., Galli, D., and Glassgold, A.E. (2009) Cosmic-ray ionization of molecular clouds. Astron Astrophys 501:619-631.

Parnell, J. (1996) Petrographic relationships between mineral phases and bitumen in the Oklo Proterozoic natural fission reactors, Gabon. Mineral Mag 60:581-593.

Parnell, J. (2004a) Mineral radioactivity in sands as a mechanism for fixation of organic carbon on the early Earth. Orig Life Evol Biosph 34:533-547.

Parnell, J. (2004b) Plate tectonics, surface mineralogy, and the early evolution of life. International Journal of Astrobiology 3:131-137.

Parnell, J., Baron, M., and Lindgren, P. (2006) Potential for irradiation of methane to form complex organic molecules in impact craters: implications for Mars, Titan and Europa. Journal of Geochemical Exploration 89:322-325.

Patel, M., Zarnecki, J., and Catling, D. (2002) Ultraviolet radiation on the surface of Mars and the Beagle $2 \mathrm{UV}$ sensor. Planet Space Sci 50:915-927.

Pavlov, A., Blinov, A., and Konstantinov, A. (2002) Sterilization of martian surface by cosmic radiation. Planet Space Sci 50: 669-673.

Pavlov, A., Pavlov, A., Mills, M., Ostryakov, V., Vasilyev, G., and Toon, O. (2005a) Catastrophic ozone loss during passage of the Solar System through an interstellar cloud. Geophys Res Lett 32:L01815.

Pavlov, A., Toon, O., Pavlov, A., Bally, J., and Pollard, D. (2005b) Passing through a giant molecular cloud: "snowball" glaciations produced by interstellar dust. Geophys Res Lett 32: L03705.

Peñate, L., Martin, O., Cardenas, R., and Agusti, S. (2010) Shortterm effects of gamma ray bursts on oceanic photosynthesis. arXiv:1007.2879v1.

Pierce, J. and Adams, P. (2009) Can cosmic rays affect cloud condensation nuclei by altering new particle formation rates? Geophys Res Lett 36:L09820.

Pigliucci, M. (2008) Is evolvability evolvable? Nat Rev Genet 9: 75-82.

Pimblott, S.M. and LaVerne, J.A. (1992) Molecular product formation in the electron radiolysis of water. Radiat Res 129: 265-271.

Prantzos, N. (2006) Li, Be, B and cosmic rays in the Galaxy. Chemical Abundances and Mixing in Stars in the Milky Way and Its Satellites, edited by S. Randich and L. Pasquini, Springer, Berlin, pp 351-357.
Prantzos, N. (2007) Origin and evolution of the light nuclides. Space Sci Rev 130:27-42.

Prantzos, N., Casse, M., and Vangioni-Flam, E. (1993) Production and evolution of $\mathrm{LiBeB}$ isotopes in the Galaxy. Astrophysical J 403:630-643.

Prinn, R. and Fegley, B. (1987) The atmospheres of Venus, Earth, and Mars: a critical comparison. Ann Rev Earth Planet Sci 15:171-212.

Radman, M., Matic, I., and Taddei, F. (1999) Evolution of evolvability. Ann NY Acad Sci 870:146-155.

Rahmstorf, S., Archer, D., Ebel, D.S., Eugster, O., Jouzel, J., Maraun, D., Neu, U., Schmidt, G.A., Severinghaus, J., Weaver, A.J., and Zachos, J. (2004) Cosmic rays, carbon dioxide and climate. Eos 85, doi:10.1029/2004EO040002.

Ramaty, R., Lingenfelter, R., and Kozlovsky, B. (2001) Spallogenic light elements and cosmic-ray origin. Space Sci Rev 99:51-60.

Rathgeber, C., Lince, M., Alric, J., Lang, A., Humphrey, E., Blankenship, R., Verméglio, A., Plumley, F., Van Dover, C., Beatty, J., and Yurkov, V. (2008) Vertical distribution and characterization of aerobic phototrophic bacteria at the Juan de Fuca Ridge in the Pacific Ocean. Photosynth Res 97:235-244.

Raulin, F., Bruston, P., Paillous, P., and Sternberg, R. (1995) The low temperature organic chemistry of Titan's geofluid. Adv Space Res 15:321-333.

Raulin, F., McKay, C., Lunine, J., and Owen, T. (2009) Titan's astrobiology. In Titan from Cassini-Huygens, edited by R.H. Brown, J.-P. Lebreton, and J.H. Waite, Springer, Dordrecht, pp 215-234.

Reedy, R., Arnold, J., and Lal, D. (1983) Cosmic-ray record in Solar System matter. Annual Review of Nuclear and Particle Science 33:505-538.

Rees, M. (1987) The origin and cosmogonic implications of seed magnetic fields. Quarterly Journal of the Royal Astronomical Society 28:197-206.

Reeves, H. (1994) On the origin of the light elements $(Z<6)$. Rev Mod Phys 66:193-216.

Reeves, H., Fowler, W.A., and Hoyle, F. (1970) Galactic cosmic ray origin of $\mathrm{Li}, \mathrm{Be}$ and $\mathrm{B}$ in Stars. Nature 226:727-729.

Reid, G., Isaksen, I., Holzer, T., and Crutzen, P. (1976) Influence of ancient solar-proton events on the evolution of life. Nature 259:177-179.

Reid, G., McAfee, J., and Crutzen, P. (1978) Effects of intense stratospheric ionisation events. Nature 275:489-492.

Reynolds, R., Squyres, S., Colburn, D., and McKay, C. (1983) On the habitability of Europa. Icarus 56:246-254.

Ricardo, A., Carrigan, M., Olcott, A., and Benner, S. (2004) Borate minerals stabilize ribose. Science 303:196.

Richmond, R., Sridhar, R., and Daly, M.J. (1999) Physicochemical survival pattern for the radiophile Deinococcus radiodurans: a polyextremophile model for life on Mars. Proc SPIE 3755:210-222.

Roboz, A. and Nagy, A. (1994) The energetics of Titan's ionosphere. J Geophys Res 99:2087-2093.

Rohde, R. and Muller, R. (2005) Cycles in fossil diversity. Nature 434:208-210.

Ruderman, M. (1974) Possible consequences of nearby supernova explosions for atmospheric ozone and terrestrial life. Science 184:1079-1081.

Ryan, J., Lockwood, J., and Debrunner, H. (2000) Solar energetic particles. Space Sci Rev 93:35-53.

Sagan, C., Thompson, W., Carlson, R., Gurnett, D., and Hord, C. (1993) A search for life on Earth from the Galileo spacecraft. Nature 365:715-721. 
Saganti, P., Cucinotta, F., Wilson, J., Simonsen, L., and Zeitlin, C. (2004) Radiation climate map for analyzing risks to astronauts on the Mars surface from galactic cosmic rays. Space Sci Rev 110:143-156.

Scalo, J. and Wheeler, J.C. (2002) Astrophysical and astrobiological implications of gamma-ray burst properties. Astrophys J 566:723-737.

Scalo, J., Kaltenegger, L., Segura, A.G., Fridlund, M., Ribas, I., Kulikov, Y.N., Grenfell, J.L., Rauer, H., Odert, P., Leitzinger, M., Selsis, F., Khodachenko, M.L., Eiroa, C., Kasting, J., and Lammer, H. (2007) M Stars as targets for terrestrial exoplanet searches and biosignature detection. Astrobiology 7:85-166.

Scherer, K., Fichtner, H., and Stawicki, O. (2002) Shielded by the wind: the influence of the interstellar medium on the environment of Earth. Journal of Atmospheric and Solar-Terrestrial Physics 64:795-804.

Scherer, K., Fichtner, H., Borrmann, T., Beer, J., Desorgher, L., Flükiger, E., Fahr, H.-J., Ferreira, S., Langner, U., Potgieter, M., Heber, B., Masarik, J., Shaviv, N., and Veizer, J. (2006) Interstellar-terrestrial relations: variable cosmic environments, the dynamic heliosphere, and their imprints on terrestrial archives and climate. Space Sci Rev 127:327-465.

Schneider, S.I. and Kasting, J.F. (2009) Radiation environments on Mars and their implications for terrestrial planetary habitability. In Bioastronomy 2007: Molecules, Microbes and Extraterrestrial Life, ASP Conference Series Vol. 420, Astronomical Society of the Pacific, San Francisco, p 149.

Schuerger, A., Richards, J., Newcombe, D., and Venkateswaran, K. (2006) Rapid inactivation of seven Bacillus spp. under simulated Mars UV irradiation. Icarus 181:52-62.

Schulze-Makuch, D. and Irwin, L.N. (2008) Life in the Universe: Expectations and Constraints, Springer-Verlag, Berlin.

Schulze-Makuch, D., Grinspoon, D., Abbas, O., Irwin, L., and Bullock, M. (2004) A sulfur-based survival strategy for putative phototrophic life in the venusian atmosphere. Astrobiology 4:11-18.

Segura, A., Kasting, J., Meadows, V., Cohen, M., Scalo, J., Crisp, D., Butler, R., and Tinetti, G. (2005) Biosignatures from Earthlike planets around M dwarfs. Astrobiology 5:706-725.

Segura, A., Walkowicz, L., Meadows, V., Kasting, J., and Hawley, S. (2010) The effect of a strong stellar flare on the atmospheric chemistry of an Earth-like planet orbiting an M dwarf. Astrobiology 10:751-771.

Setlow, P. (1994) Mechanisms which contribute to the long-term survival of spores of Bacillus species. J Appl Bacteriol 176:49S-60S.

Shaviv, N. (2002) Cosmic ray diffusion from the galactic spiral arms, iron meteorites, and a possible climatic connection. Phys Rev Lett 89, doi:10.1103/PhysRevLett.89.051102.

Shaviv, N. (2003) The spiral structure of the Milky Way, cosmic rays, and ice age epochs on Earth. New Astronomy 8:39-77.

Shaviv, N. (2005) On climate response to changes in the cosmic ray flux and radiative budget. J Geophys Res 110, doi:10.1029/ 2004JA010866.

Shaw, A.M. (2007) Astrochemistry: from Astronomy to Astrobiology, Wiley, Chichester, England.

Shea, M.A. and Smart, D.F. (2000) Cosmic ray implications for human health. Space Sci Rev 93:187-205.

Shea, M.A., Smart, D.F., McCracken, K.G., Dreschhoff, G., and Spence, H.E. (2006) Solar proton events for 450 years: the Carrington event in perspective. Adv Space Res 38:232-238.

Simakov, M., Kuzicheva, E., Antropov, A., and Dodonova, N. (2002) Abiogenic synthesis of nucleotides on the surface of small space bodies with high energy particles. Adv Space Res 30:1489-1494.
Simonsen, L., Nealy, J., Townsend, L., and Wilson, J. (1990) Radiation exposure for manned Mars surface missions. NASA Technical Paper 2979, National Aeronautics and Space Administration, Washington DC. Available online at http:// hdl.handle.net/2060/19900009041.

Simonsen, L., Nealy, J., Townsend, L., and Wilson, J. (1991) Martian regolith as space radiation shielding. J Spacecr Rockets 28:7-8.

Simonsen, L.C. and Nealy, J.E. (1993) Mars surface radiation exposure for solar maximum conditions and 1989 solar proton events. NASA Technical Paper 3300, National Aeronautics and Space Administration, Washington DC. Available online at http://hdl.handle.net/2060/19930009920.

Smith, D. and Scalo, J. (2009) Habitable zones exposed: astrosphere collapse frequency as a function of stellar mass. Astrobiology 9:673-681.

Smith, D., Scalo, J., and Wheeler, J. (2004a) Transport of ionizing radiation in terrestrial-like exoplanet atmospheres. Icarus 171:229-253.

Smith, D., Scalo, J., and Wheeler, J.C. (2004b) Importance of biologically active aurora-like ultraviolet emission: stochastic irradiation of Earth and Mars by flares and explosions. Orig Life Evol Biosph 34:513-532.

Snow, T. and Bierbaum, V. (2008) Ion chemistry in the interstellar medium. Annual Review of Analytical Chemistry 1:229-259.

Snyder, L., Lovas, F., Hollis, J., Friedel, D., Jewell, P., Remijan, A., Ilyushin, V., Alekseev, E., and and Dyubko, S. (2005) A rigorous attempt to verify interstellar glycine. Astrophys $J$ 619:914-930.

Squyres, S. and Kasting, J. (1994) Early Mars: how warm and how wet? Science 265:744-749.

Squyres, S., Grotzinger, J., Arvidson, R., Bell, J., Calvin, W., Christensen, P., Clark, B., Crisp, J., Farrand, W., Herkenhoff, K., Johnson, J., Klingelhofer, G., Knoll, A., McLennan, S., McSween, H., Morris, R., Rice, J., Rieder, R., and Soderblom, L. (2004) In situ evidence for an ancient aqueous environment at Meridiani Planum, Mars. Science 306:1709-1714.

Stevenson, D. (1999) Life-sustaining planets in interstellar space? Nature 400:32.

Stone, E., Cummings, A., McDonald, F., Heikkila, B., Lal, N., and Webber, W. (2005) Voyager 1 explores the termination shock region and the heliosheath beyond. Science 309:2017-2020.

Stone, E., Cummings, A., McDonald, F., Heikkila, B., Lal, N., and Webber, W. (2008) An asymmetric solar wind termination shock. Nature 454:71-74.

Stozhkov, Y.I. (2003) The role of cosmic rays in the atmospheric processes. J Phys G Nucl Part Phys 29, doi:10.1088/0954-3899/ $29 / 5 / 312$.

Svensmark, H. (2000) Cosmic rays and Earth's climate. Space Sci Rev 93:175-185.

Svensmark, H. (2006) Cosmic rays and the biosphere over 4 billion years. Astronomische Nachrichten 327:871-875.

Svensmark, H. (2007) Cosmoclimatology: a new theory emerges. Astronomy \& Geophysics 48:1.18-1.24.

Svensmark, H. and Friis-Christensen, E. (1997) Variation of cosmic ray flux and global cloud coverage-a missing link in solar-climate relationships. Journal of Atmospheric and SolarTerrestrial Physics 59:1225-1232.

Svensmark, H., Bondo, T., and Svensmark, J. (2009) Cosmic ray decreases affect atmospheric aerosols and clouds. Geophys Res Lett 36:L15101.

Tan, J.C. and McKee, C.F. (2004) The formation of the first stars. I. Mass infall rates, accretion disk structure, and protostellar evolution. Astrophys J 603:383-400. 
Tan, J. and McKee, C. (2008) Star formation at zero and very low metallicities. In FIRST STARS III: First Stars II Conference, AIP Conference Proceedings 990, edited by B.W. O'Shea, A. Heger, and T. Abel, American Institute of Physics, Melville, NY, pp $47-62$.

Tanaka, H. (2006) Possible terrestrial effects of a nearby supernova explosion-atmosphere's response. Journal of Atmospheric and Solar-Terrestrial Physics 68:1396-1400.

Tarduno, J., Cottrell, R., Watkeys, M., Hofmann, A., Doubrovine, P., Mamajek, E., Liu, D., Sibeck, D., Neukirch, L., and Usui, Y. (2010) Geodynamo, solar wind, and magnetopause 3.4 to 3.45 billion years ago. Science 327:1238-1240.

ten Kate, I., Garry, J., Peeters, Z., Quinn, R., Foing, B., and Ehrenfreund, P. (2005) Amino acid photostability on the martian surface. Meteorit Planet Sci 40:1185-1193.

Thomas, B. (2009) Gamma-ray bursts as a threat to life on Earth. International Journal of Astrobiology 8:183-186.

Thomas, B. and Honeyman, M. (2008) Amphibian nitrate stress as an additional terrestrial threat from astrophysical ionizing radiation events? Astrobiology 8:731-733.

Thomas, B. and Melott, A. (2006) Gamma-ray bursts and terrestrial planetary atmospheres. New J Phys 8, doi:10.1088/ 1367-2630/8/7/120.

Thomas, B., Jackman, C., and Melott, A. (2007) Modeling atmospheric effects of the September 1859 solar flare. Geophys Res Lett 34:L06810.

Thomas, B., Melott, A., Fields, B., and Anthony-Twarog, B. (2008) Superluminous supernovae: no threat from $\eta$-Carinae. Astrobiology 8:9-16.

Thomas, B.C., Jackman, C.H., Melott, A.L., Laird, C.M., Stolarski, R.S., Gehrels, N., Cannizzo, J.K., and Hogan, D.P. (2005a) Terrestrial ozone depletion due to a Milky Way gamma-ray burst. Astrophys J 622:L153-L156.

Thomas, B.C, Melott, A., Jackman, C., Laird, C., Medvedev, M., Stolarski, R., Gehrels, N., Cannizzo, J., Hogan, D., and Ejzak, L. (2005b) Gamma-ray bursts and the Earth: exploration of atmospheric, biological, climatic, and biogeochemical effects. Astrophys J 634:509-533.

Thompson, D., Wilson, P., Sims, M., Cullen, D., Holt, J., Parker, D., and Smith, M. (2006) Preliminary investigation of proton and helium ion radiation effects on fluorescent dyes for use in astrobiology applications. Anal Chem 78: 2738-2743.

Thorsett, S.E. (1995) Terrestrial implications of cosmological gamma-ray burst models. Astrophys J 444:L53-L55.

Todd, P. (2003) Space radiation health: a brief primer. Gravit Space Biol Bull 16:1-4.

United States Committee on Extension to the Standard Atmosphere NOAA. (1976) U.S. Standard Atmosphere 1976, U.S. Government Printing Office, Washington DC. Available online at http://modelweb.gsfc.nasa.gov/atmos/us_ standard.html.

Upadhyay, H. and Singh, R. (1995) Cosmic ray ionization of lower Venus atmosphere. Adv Space Res 15:99-108.

Upadhyay, H., Singh, R., and Singh, R. (1994) Cosmic ray ionization of the lower Venus atmosphere. Earth Moon Planets 65:89-94.

Usoskin, I.G. and Kovaltsov, G.A. (2006) Cosmic ray induced ionization in the atmosphere: full modeling and practical applications. J Geophys Res 111, doi:10.1029/2006JD007150.

Usoskin, I., Desorgher, L., Velinov, P., Storini, M., Flückiger, E., Bütikofer, R., and Kovaltsov, G. (2009) Ionization of the Earth's atmosphere by solar and galactic cosmic rays. Acta Geophysica 57:88-101.
Vainio, R., Desorgher, L., Heynderickx, D., Storini, M., Flückiger, E., Horne, R., Kovaltsov, G., Kudela, K., Laurenza, M., McKenna-Lawlor, S., Rothkaehl, H., and Usoskin, I. (2009) Dynamics of the Earth's particle radiation environment. Space Sci Rev 147:187-231.

Valtonen, M., Nurmi, P., Zheng, J.-Q., Cucinotta, F.A., Wilson, J.W., Horneck, G., Lindegren, L., Melosh, J., Rickman, H., and Mileikowsky, C. (2009) Natural transfer of viable microbes in space from planets in extra-solar systems to a planet in our solar system and vice versa. Astrophys $J$ 690:210.

van Dishoeck, E. and Blake, G. (2003) Chemical evolution of starforming regions. Annu Rev Astron Astrophys 36:317-368.

Van Allen, J. (1959) The geomagnetically trapped corpuscular radiation. J Geophys Res 64:1683-1689.

Vangioni-Flam, E. and Casse, M. (2000) LiBeB production and associated astrophysical sites. In The Light Elements and their Evolution, IAU Symposium 198, edited by L. da Silva, M. Spite, and R. de Medeiros, pp 1-10.

Vasilyev, G., Ostryakov, V., and Pavlov, A. (2008) Influence of energetic particles on atmospheric ionization. Journal of Atmospheric and Solar-Terrestrial Physics 70:2000-2004.

Vietri, M., Marco, D.D., and Guetta, D. (2003) On the generation of ultra-high-energy cosmic rays in gamma-ray bursts: a reappraisal. Astrophys J 592:378.

Vitousek, P. and Howarth, R. (1991) Nitrogen limitation on land and in the sea: How can it occur? Biogeochemistry 13:87115.

Vuitton, V., Lavvas, P., Yelle, R., Galand, M., Wellbrock, A., Lewis, G., Coates, A., and Wahlund, J. (2009) Negative ion chemistry in Titan's upper atmosphere. Planet Space Sci 57: 1558-1572.

Wackett, L., Dodge, A., and Ellis, L. (2004) Microbial genomics and the periodic table. Appl Environ Microbiol 70:647655.

Wahlund, J.-E., Boström, R., Gustafsson, G., Gurnett, D., Kurth, W., Pedersen, A., Averkamp, T., Hospodarsky, G., Persoon, A., Canu, P., Neubauer, F., Dougherty, M., Eriksson, A., Morooka, M., Gill, R., André, M., Eliasson, L., and MüllerWodarg, I. (2005) Cassini measurements of cold plasma in the ionosphere of Titan. Science 308:986-989.

Waite, J., Jr., Young, D., and Cravens, T. (2007) The process of tholin formation in Titan's upper atmosphere. Science 870: 870-875.

Webber, W. and Intriligator, D. (2011) Voyagers 1 and 2 in a shrunken and squashed heliosphere. J Geophys Res 116, doi:10.1029/2011JA016478.

Wells, L.E., Armstrong, J.C., and Gonzalez, G. (2003) Reseeding of early Earth by impacts of returning ejecta during the Late Heavy Bombardment. Icarus 162:38-46.

White, S., Chave, A., and Reynolds, G. (2002) Investigations of ambient light emission at deep-sea hydrothermal vents. J Geophys Res 107:1-13.

Whitten, R., Cuzzi, J., Borucki, W.J., and Wolfe, J.H. (1976) Effect of nearby supernova explosions on atmospheric ozone. Nature 263:398-400.

Williams, D. and Herbst, E. (2002) It's a dusty Universe: surface science in space. Surf Sci 500:823-837.

Wilson, E. and Atreya, S. (2004) Current state of modeling the photochemistry of Titan's mutually dependent atmosphere and ionosphere. J Geophys Res 109, doi:10.1029/2003JE002181.

Witasse, O., Cravens, T., Mendillo, M., Moses, J., Kliore, A., Nagy, A., and Breus, T. (2008) Solar system ionospheres. Space Sci Rev 139:235-265. 
Yamazaki, T. and Oda, H. (2002) Orbital influence on Earth's magnetic field: 100,000-year periodicity in inclination. Science 295:2435-2438.

Yeghikyan, A. and Fahr, H. (2004a) Effects induced by the passage of the Sun through dense molecular clouds. Astron Astrophys 415:763-770.

Yeghikyan, A. and Fahr, H. (2004b) Terrestrial atmospheric effects induced by counterstreaming dense interstellar cloud material. Astron Astrophys 425:1113-1118.

Yelle, R.V., Strobell, D.F., Lellouch, E., and Gautier, D. (1997) Engineering models for Titan's atmosphere. In Huygens: Science, Payload and Mission, Proceedings of an ESA conference, ESA SP-1177, edited by A. Wilson, pp 243-256.

Yen, A., Kim, S., Hecht, M., Frant, M., and Murray, B. (2000) Evidence that the reactivity of the martian soil is due to superoxide ions. Science 289:1909-1912.

Zank, G. and Frisch, P. (1999) Consequences of a change in the galactic environment of the Sun. Astrophys J 518:965-973.

Zent, A.P. and McKay, C.P. (1994) The chemical reactivity of the martian soil and implications for future missions. Icarus 108:146-157.
Zetterström, L. (2000) Oklo: a review and critical evaluation of literature. Technical Report TR-00-17, Swedish Nuclear Fuel and Waste Management Co., Stockholm. Available online at http:// www.skb.se/upload/publications/pdf/Tr-00-17webb.pdf.

Zhai, M. (1995) Boron cosmochemistry. Part II: Boron nucleosynthesis and condensation temperature. Meteoritics 30:733-737.

Zweibel, E.C. and Heiles, C. (1997) Magnetic fields in galaxies and beyond. Nature 385:131-136.

Address correspondence to:

Lewis R. Dartnell

The Centre for Planetary Sciences

University College London

Gower Street

London WC1E 6BT

UK

E-mail: 1.dartnell@ucl.ac.uk

Submitted 18 March 2011

Accepted 1 May 2011 